\title{
Dieckmann Condensation of Ugi $N$-Acylamino Amide Product: Facile Access to Functionalized 2,2-Disubstituted Indolin-3-ones
}

Yong Li, ${ }^{\dagger}, "$ Jia Xu, ${ }^{\dagger}, "$ Liu-Jun He, ${ }^{\dagger}$ Ya-Fei Luo, ${ }^{\dagger}$ Jiang-Ping Meng, ${ }^{\dagger}$ Dian-Yong Tang,

${ }^{\dagger}$ Hong-yu Li, ${ }^{\ddagger}, *$ Zhong-Zhu Chen ${ }^{\dagger}, *$ Zhi-Gang Xu, ${ }^{\dagger}, *$

${ }^{\dagger}$ College of Pharmacy, National \& Local Joint Engineering Research Center of Targeted and Innovative Therapeutics, IATTI, Chongqing University of Arts and Sciences. 319 Honghe Ave., Yongchuan, Chongqing, 402160, China

${ }^{\ddagger}$ Department of Pharmaceutical Sciences, College of Pharmacy, University of Arkansas for Medical Sciences, Little Rock, AR 72205, USA

These authors contributed equally to this work.

\section{Table of Content}

1. Density functional theory (DFT) calculations S2

2. X-Ray Analysis of $\mathbf{6 h}$ and $\mathbf{1 0 e}$

3. NMR Spectra. S31 
Density functional theory (DFT) calculations
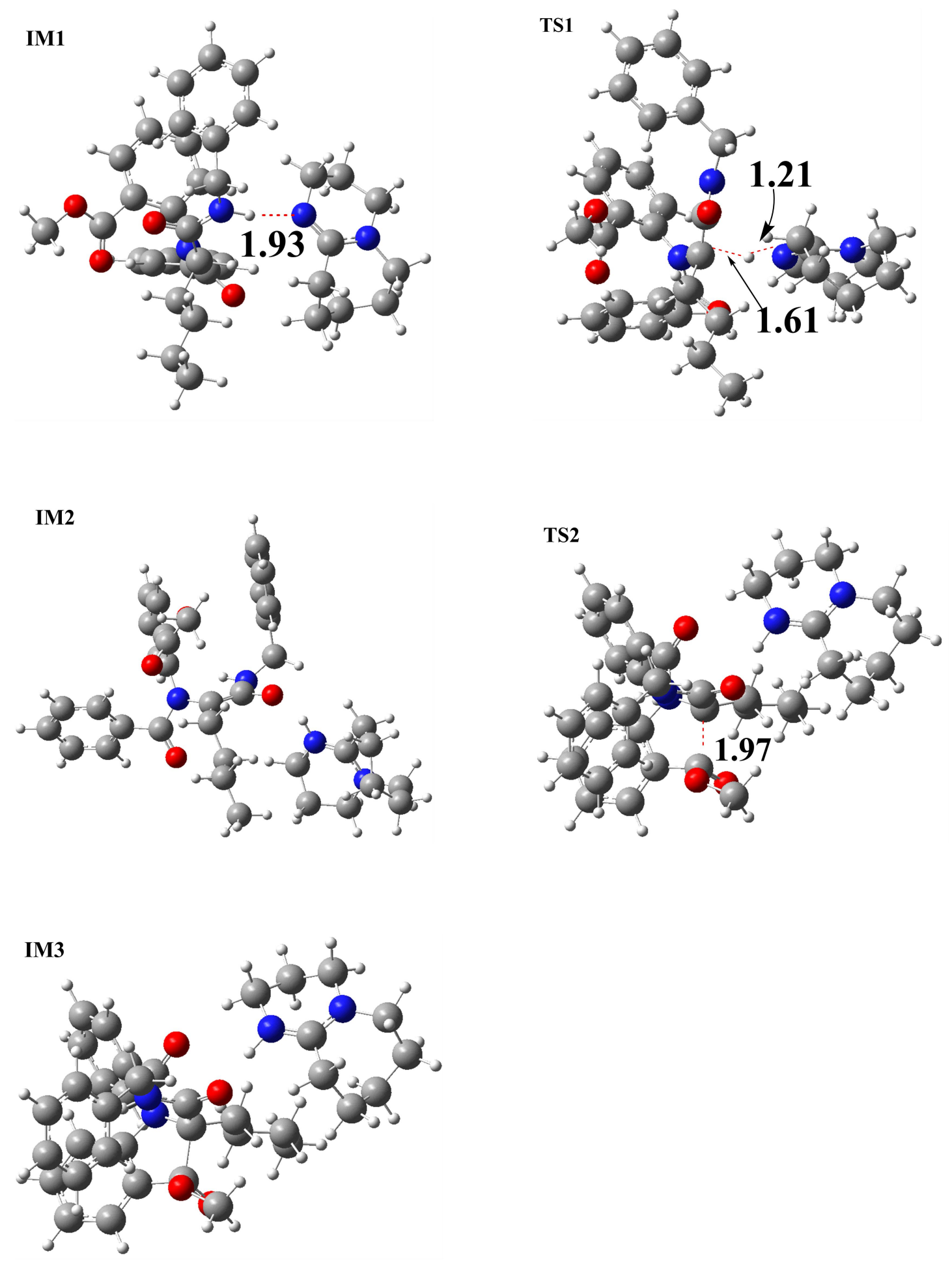

Fig. S1 Calculated activation modes and potential energy profiles of the Dieckmann condensation reaction. 


\section{Pathyway 1:}

\section{IM1}

Sum of electronic and zero-point Energies: -1957.321212 a.u.

\begin{tabular}{|c|c|c|c|}
\hline \multirow{2}{*}{$\begin{array}{l}\text { Atomic } \\
\text { Number }\end{array}$} & \multicolumn{3}{|c|}{ Coordinates (Angstroms) } \\
\hline & $\mathrm{X}$ & $\mathrm{Y}$ & $\mathrm{Z}$ \\
\hline 6 & -4.250764 & 10.386318 & 2.049654 \\
\hline 6 & -3.840001 & 9.050330 & 1.414120 \\
\hline 6 & -3.182858 & 9.138366 & 0.028342 \\
\hline 6 & -1.899241 & 10.016698 & -0.060839 \\
\hline 6 & -1.141866 & 10.188105 & 1.240336 \\
\hline 7 & -1.787473 & 10.898938 & 2.226531 \\
\hline 6 & -3.128242 & 11.445650 & 2.011846 \\
\hline 7 & 0.041727 & 9.677873 & 1.341663 \\
\hline 6 & 0.760873 & 9.872452 & 2.599482 \\
\hline 6 & -0.179365 & 9.905814 & 3.804267 \\
\hline 6 & -1.236230 & 10.982448 & 3.583471 \\
\hline 1 & -4.556088 & 10.203500 & 3.085822 \\
\hline 1 & -5.126851 & 10.803316 & 1.539480 \\
\hline 1 & -3.150411 & 8.530349 & 2.085371 \\
\hline 1 & -4.718431 & 8.402517 & 1.341270 \\
\hline 1 & -3.907433 & 9.505858 & -0.706625 \\
\hline 1 & -2.927133 & 8.117364 & -0.260974 \\
\hline 1 & -2.149114 & 11.009207 & -0.448655 \\
\hline 1 & -1.199846 & 9.580668 & -0.772782 \\
\hline 1 & -3.296927 & 12.206676 & 2.777585 \\
\hline 1 & -3.148357 & 11.974224 & 1.057006 \\
\hline 1 & 1.496353 & 9.070403 & 2.701405 \\
\hline 1 & 1.334381 & 10.809218 & 2.552448 \\
\hline 1 & 0.366458 & 10.104508 & 4.730565 \\
\hline 1 & -0.664462 & 8.929966 & 3.908742 \\
\hline 1 & -0.805115 & 11.979334 & 3.747335 \\
\hline 1 & -2.055812 & 10.864265 & 4.299458 \\
\hline 6 & -2.109527 & 1.868403 & 3.805901 \\
\hline 6 & -1.280776 & 1.652294 & 2.707001 \\
\hline 6 & -0.955337 & 2.703922 & 1.853663 \\
\hline 6 & -1.472631 & 3.981003 & 2.092576 \\
\hline 6 & -2.334289 & 4.185548 & 3.175000 \\
\hline 6 & -2.637578 & 3.138305 & 4.037992 \\
\hline 1 & -2.353536 & 1.048607 & 4.472574 \\
\hline 1 & -0.889113 & 0.660735 & 2.509087 \\
\hline 1 & -0.330808 & 2.533847 & 0.985129 \\
\hline
\end{tabular}




\begin{tabular}{|c|c|c|c|}
\hline 1 & -2.767086 & 5.167648 & 3.322836 \\
\hline 1 & -3.294060 & 3.307708 & 4.884312 \\
\hline 6 & -1.243192 & 5.120156 & 1.139201 \\
\hline 8 & -2.179370 & 5.840788 & 0.812629 \\
\hline 7 & 0.026986 & 5.285346 & 0.608182 \\
\hline 6 & 1.179490 & 4.921054 & 1.384412 \\
\hline 6 & 2.188310 & 4.054882 & 0.920977 \\
\hline 6 & 1.313452 & 5.492023 & 2.653667 \\
\hline 6 & 3.286909 & 3.785204 & 1.744949 \\
\hline 6 & 2.394591 & 5.192351 & 3.474420 \\
\hline 6 & 3.388134 & 4.332976 & 3.018031 \\
\hline 1 & 4.059918 & 3.126585 & 1.372349 \\
\hline 1 & 2.464644 & 5.640844 & 4.458648 \\
\hline 1 & 4.240471 & 4.095863 & 3.643865 \\
\hline 6 & 2.099116 & 3.361345 & -0.400407 \\
\hline 8 & 1.075539 & 2.986239 & -0.924107 \\
\hline 8 & 3.319968 & 3.130852 & -0.916597 \\
\hline 6 & 3.340219 & 2.514794 & -2.214705 \\
\hline 1 & 2.772431 & 1.583526 & -2.210682 \\
\hline 1 & 2.914849 & 3.197438 & -2.951496 \\
\hline 1 & 4.389862 & 2.328564 & -2.431359 \\
\hline 6 & 0.112588 & 6.295215 & -0.494414 \\
\hline 6 & 1.551382 & 6.770344 & -0.759668 \\
\hline 1 & 1.099656 & 8.576609 & 0.154597 \\
\hline 6 & 3.059224 & 8.706540 & -0.711012 \\
\hline 1 & 2.826427 & 9.743186 & -0.967561 \\
\hline 1 & 3.452723 & 8.213946 & -1.602567 \\
\hline 6 & -0.496106 & 5.741422 & -1.796175 \\
\hline 1 & 0.136156 & 4.926867 & -2.150304 \\
\hline 1 & -1.474456 & 5.320557 & -1.555605 \\
\hline 6 & -0.659457 & 6.804889 & -2.887678 \\
\hline 1 & -1.272754 & 7.633062 & -2.508615 \\
\hline 1 & 0.317318 & 7.232295 & -3.143281 \\
\hline 6 & -1.305928 & 6.248391 & -4.162037 \\
\hline 1 & -0.696104 & 5.419249 & -4.539388 \\
\hline 1 & -2.282897 & 5.817951 & -3.912075 \\
\hline 6 & -1.476329 & 7.299642 & -5.262337 \\
\hline 1 & -1.937081 & 6.871538 & -6.157050 \\
\hline 1 & -2.110964 & 8.125789 & -4.925693 \\
\hline 1 & -0.511154 & 7.723991 & -5.556593 \\
\hline 8 & 2.366229 & 6.060186 & -1.345156 \\
\hline 7 & 1.802877 & 8.040445 & -0.374713 \\
\hline 6 & 4.105628 & 8.680957 & 0.388278 \\
\hline 6 & 4.485120 & 9.856140 & 1.040507 \\
\hline
\end{tabular}




$\begin{array}{rrrr}6 & 4.721642 & 7.477565 & 0.753115 \\ 6 & 5.459000 & 9.837757 & 2.038881 \\ 1 & 4.018312 & 10.795878 & 0.761463 \\ 6 & 5.692366 & 7.457593 & 1.750082 \\ 1 & 4.433985 & 6.562820 & 0.247150 \\ 6 & 6.064985 & 8.636675 & 2.396242 \\ 1 & 5.744510 & 10.760344 & 2.532715 \\ 1 & 6.163944 & 6.519137 & 2.020182 \\ 1 & 6.825074 & 8.618321 & 3.169601 \\ 1 & 0.550183 & 6.182544 & 2.991063 \\ 1 & -0.479651 & 7.148207 & -0.157417\end{array}$

TS1

Sum of electronic and zero-point Energies: -1957.265855 a.u.

\begin{tabular}{|c|c|c|c|}
\hline \multirow{2}{*}{$\begin{array}{l}\text { Atomic } \\
\text { Number }\end{array}$} & \multicolumn{3}{|c|}{ Coordinates (Angstroms) } \\
\hline & $\mathrm{X}$ & $\mathrm{Y}$ & $\mathrm{Z}$ \\
\hline 6 & -3.401055 & 12.036275 & -0.614411 \\
\hline 6 & -3.265869 & 10.769576 & -1.472139 \\
\hline 6 & -1.823426 & 10.388071 & -1.835359 \\
\hline 6 & -0.867076 & 10.056195 & -0.648565 \\
\hline 6 & -1.528444 & 9.764688 & 0.678877 \\
\hline 7 & -2.269979 & 10.761660 & 1.230622 \\
\hline 6 & -2.407235 & 12.060329 & 0.560161 \\
\hline 7 & -1.349081 & 8.613945 & 1.270996 \\
\hline 6 & -1.924135 & 8.363960 & 2.591940 \\
\hline 6 & -3.258611 & 9.083320 & 2.741660 \\
\hline 6 & -3.068150 & 10.565131 & 2.450879 \\
\hline 1 & -4.427791 & 12.111778 & -0.240921 \\
\hline 1 & -3.224634 & 12.931236 & -1.221368 \\
\hline 1 & -3.732193 & 9.928938 & -0.948010 \\
\hline 1 & -3.841073 & 10.899585 & -2.393609 \\
\hline 1 & -1.369860 & 11.188261 & -2.429161 \\
\hline 1 & -1.863321 & 9.513861 & -2.488354 \\
\hline 1 & -0.186048 & 10.896024 & -0.476235 \\
\hline 1 & -0.221014 & 9.220529 & -0.913585 \\
\hline 1 & -2.719530 & 12.783184 & 1.316785 \\
\hline 1 & -1.425338 & 12.386049 & 0.214821 \\
\hline 1 & -2.046686 & 7.285904 & 2.680542 \\
\hline 1 & -1.218161 & 8.703147 & 3.361730 \\
\hline 1 & -3.660258 & 8.951900 & 3.749108 \\
\hline 1 & -3.977628 & 8.650442 & 2.040378 \\
\hline 1 & -2.568631 & 11.063936 & 3.290808 \\
\hline
\end{tabular}




\begin{tabular}{|c|c|c|c|}
\hline 1 & -4.033660 & 11.058443 & 2.306455 \\
\hline 6 & -0.890384 & 1.163734 & 4.026485 \\
\hline 6 & -0.147532 & 1.277325 & 2.853595 \\
\hline 6 & -0.078244 & 2.493213 & 2.177156 \\
\hline 6 & -0.767400 & 3.607574 & 2.667723 \\
\hline 6 & -1.544405 & 3.476614 & 3.824105 \\
\hline 6 & -1.591081 & 2.268233 & 4.510438 \\
\hline 1 & -0.934433 & 0.217354 & 4.554244 \\
\hline 1 & 0.378622 & 0.415338 & 2.458470 \\
\hline 1 & 0.486343 & 2.570589 & 1.255902 \\
\hline 1 & -2.117935 & 4.328076 & 4.170793 \\
\hline 1 & -2.183347 & 2.182962 & 5.414979 \\
\hline 6 & -0.830517 & 4.912091 & 1.915543 \\
\hline 8 & -1.930088 & 5.433435 & 1.738719 \\
\hline 7 & 0.332225 & 5.452833 & 1.400483 \\
\hline 6 & 1.599518 & 5.035631 & 1.921113 \\
\hline 6 & 2.648997 & 4.559808 & 1.112300 \\
\hline 6 & 1.842380 & 5.204913 & 3.290702 \\
\hline 6 & 3.900131 & 4.305271 & 1.678707 \\
\hline 6 & 3.078690 & 4.902076 & 3.852512 \\
\hline 6 & 4.120278 & 4.462222 & 3.043195 \\
\hline 1 & 4.701562 & 3.963311 & 1.036004 \\
\hline 1 & 3.230552 & 5.035246 & 4.917651 \\
\hline 1 & 5.096309 & 4.252489 & 3.463495 \\
\hline 6 & 2.444345 & 4.184840 & -0.319943 \\
\hline 8 & 1.567783 & 3.453896 & -0.716217 \\
\hline 8 & 3.406566 & 4.687920 & -1.116304 \\
\hline 6 & 3.309342 & 4.336393 & -2.511066 \\
\hline 1 & 3.325051 & 3.252537 & -2.632967 \\
\hline 1 & 2.387595 & 4.735891 & -2.933796 \\
\hline 1 & 4.175472 & 4.792768 & -2.984385 \\
\hline 6 & 0.211143 & 6.464709 & 0.317290 \\
\hline 6 & 1.301550 & 7.415835 & 0.126024 \\
\hline 1 & 2.019211 & 7.338592 & 2.077853 \\
\hline 6 & 2.997273 & 8.946324 & 1.110852 \\
\hline 1 & 2.789045 & 9.698176 & 1.881910 \\
\hline 1 & 2.813529 & 9.404494 & 0.137099 \\
\hline 6 & -0.389086 & 5.882689 & -0.974311 \\
\hline 1 & 0.298136 & 6.097813 & -1.799518 \\
\hline 1 & -0.433659 & 4.795649 & -0.876625 \\
\hline 6 & -1.787618 & 6.379676 & -1.378823 \\
\hline 1 & -2.481582 & 6.219065 & -0.549139 \\
\hline 1 & -1.756621 & 7.458997 & -1.565117 \\
\hline 6 & -2.314816 & 5.678856 & -2.636143 \\
\hline
\end{tabular}




$\begin{array}{lrrr}1 & -1.606729 & 5.828752 & -3.461133 \\ 1 & -2.344182 & 4.597355 & -2.456564 \\ 6 & -3.705351 & 6.157585 & -3.064717 \\ 1 & -4.057049 & 5.630932 & -3.957040 \\ 1 & -4.440144 & 5.992138 & -2.270273 \\ 1 & -3.703496 & 7.228846 & -3.293651 \\ 8 & 1.472428 & 7.976784 & -0.975092 \\ 7 & 2.036797 & 7.860761 & 1.217760 \\ 6 & 4.454437 & 8.530744 & 1.210557 \\ 6 & 5.292833 & 9.089774 & 2.177003 \\ 6 & 4.990925 & 7.599538 & 0.313355 \\ 6 & 6.640380 & 8.735918 & 2.249740 \\ 1 & 4.888174 & 9.811653 & 2.880556 \\ 6 & 6.334565 & 7.245516 & 0.382971 \\ 1 & 4.346853 & 7.151282 & -0.434054 \\ 6 & 7.165419 & 7.812701 & 1.350497 \\ 1 & 7.276144 & 9.180404 & 3.008035 \\ 1 & 6.736865 & 6.525659 & -0.321892 \\ 1 & 8.212721 & 7.535511 & 1.401627 \\ 1 & 1.042614 & 5.587222 & 3.913732 \\ 1 & -0.714350 & 7.686770 & 0.810187\end{array}$

\section{IM2}

Sum of electronic and zero-point Energies: -1957.269935 a.u.

\begin{tabular}{cccc} 
Atomic & \multicolumn{3}{c}{ Coordinates (Angstroms) } \\
Number & $\mathrm{X}$ & $\mathrm{Y}$ & $\mathrm{Z}$ \\
& & & \\
\hline 6 & -5.533761 & 9.265761 & 3.127966 \\
6 & -4.565006 & 8.830189 & 2.019042 \\
6 & -3.183422 & 9.498531 & 2.042437 \\
6 & -2.325043 & 9.290570 & 3.328534 \\
6 & -2.742421 & 8.139728 & 4.204074 \\
7 & -3.969999 & 8.142717 & 4.746390 \\
6 & -4.878561 & 9.278251 & 4.520266 \\
7 & -1.891272 & 7.164941 & 4.437932 \\
6 & -2.217577 & 5.982183 & 5.239061 \\
6 & -3.715561 & 5.720801 & 5.163821 \\
\hline 6 & & &
\end{tabular}




\begin{tabular}{|c|c|c|c|}
\hline 6 & -4.486559 & 6.990051 & 5.507149 \\
\hline 1 & -6.402389 & 8.599233 & 3.128661 \\
\hline 1 & -5.915835 & 10.274025 & 2.934423 \\
\hline 1 & -4.430843 & 7.745547 & 2.066927 \\
\hline 1 & -5.028698 & 9.022580 & 1.047425 \\
\hline 1 & -3.287031 & 10.574959 & 1.871848 \\
\hline 1 & -2.591038 & 9.091829 & 1.221093 \\
\hline 1 & -2.326391 & 10.191368 & 3.951298 \\
\hline 1 & -1.305990 & 9.100558 & 2.996823 \\
\hline 1 & -5.632116 & 9.246535 & 5.309144 \\
\hline 1 & -4.318142 & 10.202615 & 4.666358 \\
\hline 1 & -1.671454 & 5.142883 & 4.810095 \\
\hline 1 & -1.893743 & 6.144046 & 6.274051 \\
\hline 1 & -3.996031 & 4.920631 & 5.851118 \\
\hline 1 & -3.961240 & 5.388399 & 4.152439 \\
\hline 1 & -4.420804 & 7.213007 & 6.578822 \\
\hline 1 & -5.544275 & 6.877446 & 5.258176 \\
\hline 6 & 0.830929 & -0.725867 & 3.102292 \\
\hline 6 & 1.121721 & -0.023192 & 1.934740 \\
\hline 6 & 0.778865 & 1.321549 & 1.816927 \\
\hline 6 & 0.127896 & 1.972854 & 2.871514 \\
\hline 6 & -0.193822 & 1.251762 & 4.027149 \\
\hline 6 & 0.169784 & -0.084405 & 4.149825 \\
\hline 1 & 1.107129 & -1.770809 & 3.192178 \\
\hline 1 & 1.614332 & -0.523552 & 1.108361 \\
\hline 1 & 0.992161 & 1.858354 & 0.900021 \\
\hline 1 & -0.740070 & 1.751257 & 4.818303 \\
\hline 1 & -0.070639 & -0.629856 & 5.055969 \\
\hline 6 & -0.357873 & 3.391766 & 2.757541 \\
\hline 8 & -1.460109 & 3.685948 & 3.219974 \\
\hline
\end{tabular}




\begin{tabular}{|c|c|c|c|}
\hline 7 & 0.421606 & 4.305953 & 2.060651 \\
\hline 6 & 1.845130 & 4.189994 & 2.067598 \\
\hline 6 & 2.619924 & 4.377962 & 0.907285 \\
\hline 6 & 2.509430 & 3.990276 & 3.284824 \\
\hline 6 & 4.011616 & 4.396097 & 0.996680 \\
\hline 6 & 3.900035 & 3.964598 & 3.355013 \\
\hline 6 & 4.658732 & 4.182171 & 2.211696 \\
\hline 1 & 4.588188 & 4.562539 & 0.095340 \\
\hline 1 & 4.384357 & 3.802905 & 4.311590 \\
\hline 1 & 5.740872 & 4.194588 & 2.260475 \\
\hline 6 & 2.000092 & 4.403406 & -0.451403 \\
\hline 8 & 1.240575 & 3.559030 & -0.867738 \\
\hline 8 & 2.472865 & 5.409772 & -1.211364 \\
\hline 6 & 1.979996 & 5.448462 & -2.563052 \\
\hline 1 & 2.212634 & 4.517876 & -3.083221 \\
\hline 1 & 0.900370 & 5.600981 & -2.566931 \\
\hline 1 & 2.486328 & 6.289415 & -3.031800 \\
\hline 6 & -0.201873 & 5.444757 & 1.411632 \\
\hline 6 & -0.018843 & 6.743980 & 1.863572 \\
\hline 1 & 1.237476 & 6.161066 & 3.406065 \\
\hline 6 & 1.477468 & 8.205427 & 3.240645 \\
\hline 1 & 1.665179 & 8.382995 & 4.304474 \\
\hline 1 & 0.843139 & 9.010689 & 2.869742 \\
\hline 6 & -1.172793 & 5.179203 & 0.283176 \\
\hline 1 & -0.975074 & 5.905354 & -0.516428 \\
\hline 1 & -0.967771 & 4.191592 & -0.138045 \\
\hline 6 & -2.673992 & 5.274283 & 0.628445 \\
\hline 1 & -2.910262 & 4.552975 & 1.416066 \\
\hline 1 & -2.869694 & 6.272695 & 1.035147 \\
\hline 6 & -3.575300 & 5.030779 & -0.587233 \\
\hline
\end{tabular}




\begin{tabular}{|c|c|c|c|}
\hline 1 & -3.337939 & 5.764320 & -1.368265 \\
\hline 1 & -3.341684 & 4.048218 & -1.015997 \\
\hline 6 & -5.072223 & 5.095112 & -0.267252 \\
\hline 1 & -5.685424 & 4.907099 & -1.154135 \\
\hline 1 & -5.345155 & 4.350410 & 0.487814 \\
\hline 1 & -5.351730 & 6.079464 & 0.123668 \\
\hline 8 & -0.493575 & 7.784687 & 1.324095 \\
\hline 7 & 0.684360 & 6.963819 & 3.128174 \\
\hline 6 & 2.794670 & 8.237308 & 2.489124 \\
\hline 6 & 4.011412 & 8.207255 & 3.175577 \\
\hline 6 & 2.811851 & 8.329795 & 1.091059 \\
\hline 6 & 5.223157 & 8.272142 & 2.489255 \\
\hline 1 & 4.011313 & 8.140173 & 4.259744 \\
\hline 6 & 4.020974 & 8.390163 & 0.404953 \\
\hline 1 & 1.868455 & 8.352243 & 0.557332 \\
\hline 6 & 5.229900 & 8.365133 & 1.100036 \\
\hline 1 & 6.158201 & 8.252748 & 3.038984 \\
\hline 1 & 4.019524 & 8.458361 & -0.677225 \\
\hline 1 & 6.170216 & 8.418157 & 0.561857 \\
\hline 1 & 1.921456 & 3.859707 & 4.186012 \\
\hline 1 & -0.953659 & 7.166152 & 3.972638 \\
\hline
\end{tabular}

\section{TS2}

Sum of electronic and zero-point Energies: -1957.267629 a.u.

\begin{tabular}{cccc} 
Atomic & \multicolumn{3}{c}{ Coordinates (Angstroms) } \\
Number & $X$ & Y & $Z$ \\
\hline--------- \\
6 & -4.463639 & 11.168323 & -2.168596 \\
6 & -3.514293 & 10.002093 & -2.483235 \\
6 & -2.022237 & 10.356982 & -2.573051 \\
6 & -1.390577 & 11.001911 & -1.301368 \\
6 & -2.153977 & 10.754678 & -0.024874 \\
7 & -3.394419 & 11.249064 & 0.109385
\end{tabular}




\begin{tabular}{|c|c|c|c|}
\hline 6 & -4.001495 & 12.031734 & -0.978188 \\
\hline 7 & -1.573049 & 10.062992 & 0.924308 \\
\hline 6 & -2.224311 & 9.703227 & 2.186587 \\
\hline 6 & -3.734872 & 9.666581 & 1.981837 \\
\hline 6 & -4.208343 & 10.949466 & 1.302681 \\
\hline 1 & -5.461823 & 10.765341 & -1.970169 \\
\hline 1 & -4.563215 & 11.828452 & -3.036856 \\
\hline 1 & -3.643320 & 9.221239 & -1.727382 \\
\hline 1 & -3.820027 & 9.544233 & -3.427819 \\
\hline 1 & -1.846504 & 11.028249 & -3.418981 \\
\hline 1 & -1.474966 & 9.437146 & -2.787336 \\
\hline 1 & -1.304873 & 12.085397 & -1.426593 \\
\hline 1 & -0.381352 & 10.617755 & -1.158645 \\
\hline 1 & -4.839599 & 12.582521 & -0.548778 \\
\hline 1 & -3.281059 & 12.781189 & -1.309803 \\
\hline 1 & -1.837778 & 8.728197 & 2.487582 \\
\hline 1 & -1.949812 & 10.437762 & 2.952106 \\
\hline 1 & -4.244834 & 9.549450 & 2.939734 \\
\hline 1 & -3.993219 & 8.802006 & 1.364581 \\
\hline 1 & -4.154849 & 11.799360 & 1.993062 \\
\hline 1 & -5.246900 & 10.851968 & 0.979415 \\
\hline 6 & -1.801883 & 2.140495 & 4.518338 \\
\hline 6 & -1.560776 & 2.043211 & 3.148856 \\
\hline 6 & -1.091810 & 3.145269 & 2.439743 \\
\hline 6 & -0.875805 & 4.362519 & 3.094806 \\
\hline 6 & -1.149451 & 4.462421 & 4.462673 \\
\hline 6 & -1.593281 & 3.352706 & 5.174921 \\
\hline 1 & -2.156931 & 1.277155 & 5.070150 \\
\hline 1 & -1.735281 & 1.106767 & 2.631168 \\
\hline 1 & -0.897187 & 3.063916 & 1.377804 \\
\hline 1 & -1.014882 & 5.418695 & 4.953470 \\
\hline 1 & -1.785901 & 3.434971 & 6.238900 \\
\hline 6 & -0.493458 & 5.612519 & 2.352111 \\
\hline 8 & -1.090664 & 6.656931 & 2.617305 \\
\hline 7 & 0.439131 & 5.561074 & 1.338381 \\
\hline 6 & 1.510975 & 4.631241 & 1.176170 \\
\hline 6 & 2.160015 & 4.753388 & -0.052387 \\
\hline 6 & 2.001479 & 3.742883 & 2.141883 \\
\hline 6 & 3.244084 & 3.936318 & -0.353548 \\
\hline 6 & 3.089576 & 2.931773 & 1.828116 \\
\hline 6 & 3.705793 & 3.011755 & 0.579707 \\
\hline 1 & 3.726412 & 4.044434 & -1.318062 \\
\hline 1 & 3.463327 & 2.240803 & 2.575800 \\
\hline 1 & 4.554210 & 2.377340 & 0.348674 \\
\hline
\end{tabular}




\begin{tabular}{|c|c|c|c|}
\hline 6 & 1.620829 & 5.798498 & -1.018666 \\
\hline 8 & 0.969915 & 5.453439 & -2.022298 \\
\hline 8 & 2.687148 & 6.760267 & -1.207000 \\
\hline 6 & 2.570068 & 7.563021 & -2.375020 \\
\hline 1 & 2.262887 & 6.959272 & -3.231022 \\
\hline 1 & 1.846578 & 8.373443 & -2.229969 \\
\hline 1 & 3.556841 & 7.996559 & -2.551886 \\
\hline 6 & 0.423117 & 6.615766 & 0.317014 \\
\hline 6 & 1.051979 & 7.862716 & 0.767504 \\
\hline 1 & 2.308346 & 6.885041 & 2.041054 \\
\hline 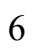 & 2.839562 & 8.934514 & 2.107053 \\
\hline 1 & 2.548206 & 9.273398 & 3.109573 \\
\hline 1 & 2.592983 & 9.740956 & 1.412698 \\
\hline 6 & -0.903860 & 6.794366 & -0.415426 \\
\hline 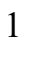 & -1.538815 & 7.529904 & 0.089885 \\
\hline 1 & -0.652483 & 7.197114 & -1.401835 \\
\hline 6 & -1.719182 & 5.513569 & -0.634216 \\
\hline 1 & -1.060822 & 4.744232 & -1.042332 \\
\hline 1 & -2.119949 & 5.150203 & 0.318991 \\
\hline 6 & -2.889315 & 5.729129 & -1.600680 \\
\hline 1 & -3.529521 & 6.542612 & -1.230004 \\
\hline 1 & -2.492496 & 6.062133 & -2.566759 \\
\hline 6 & -3.740146 & 4.472380 & -1.807199 \\
\hline 1 & -4.562464 & 4.650245 & -2.507148 \\
\hline 1 & -3.133806 & 3.654621 & -2.207807 \\
\hline 1 & -4.174893 & 4.128735 & -0.862814 \\
\hline 8 & 0.747196 & 8.986670 & 0.276725 \\
\hline 7 & 2.032817 & 7.794618 & 1.704949 \\
\hline 6 & 4.330287 & 8.653543 & 2.092765 \\
\hline 6 & 5.147520 & 9.177187 & 3.098380 \\
\hline 6 & 4.914384 & 7.901963 & 1.066292 \\
\hline 6 & 6.525200 & 8.969471 & 3.078966 \\
\hline 1 & 4.701624 & 9.750077 & 3.906233 \\
\hline 6 & 6.291164 & 7.689401 & 1.052832 \\
\hline 1 & 4.287238 & 7.477331 & 0.288606 \\
\hline 6 & 7.101421 & 8.223311 & 2.053787 \\
\hline 1 & 7.144854 & 9.381469 & 3.868234 \\
\hline 1 & 6.731275 & 7.100653 & 0.255330 \\
\hline 1 & 8.172416 & 8.053084 & 2.038918 \\
\hline 1 & 1.554771 & 3.677754 & 3.123248 \\
\hline & -0.634768 & 9.629462 & 0.699493 \\
\hline
\end{tabular}




\section{IM3}

Sum of electronic and zero-point Energies: -1957.268749 a.u.

\begin{tabular}{|c|c|c|c|}
\hline \multirow{2}{*}{$\begin{array}{l}\text { Atomic } \\
\text { Number }\end{array}$} & \multicolumn{3}{|c|}{ Coordinates (Angstroms) } \\
\hline & $\mathrm{X}$ & $\mathrm{Y}$ & $\mathrm{Z}$ \\
\hline 6 & -4.358292 & 11.156746 & -2.274866 \\
\hline 6 & -3.388539 & 9.999548 & -2.559449 \\
\hline 6 & -1.895497 & 10.360846 & -2.559839 \\
\hline 6 & -1.336834 & 10.978839 & -1.241225 \\
\hline 6 & -2.170781 & 10.708228 & -0.014859 \\
\hline 7 & -3.416316 & 11.199121 & 0.059488 \\
\hline 6 & -3.963494 & 12.000440 & -1.046969 \\
\hline 7 & -1.643523 & 10.000863 & 0.956068 \\
\hline 6 & -2.362496 & 9.623686 & 2.176022 \\
\hline 6 & -3.858846 & 9.586485 & 1.884979 \\
\hline 6 & -4.296254 & 10.878756 & 1.199569 \\
\hline 1 & -5.364065 & 10.747474 & -2.136931 \\
\hline 1 & -4.414200 & 11.832303 & -3.135070 \\
\hline 1 & -3.556028 & 9.202960 & -1.827960 \\
\hline 1 & -3.639839 & 9.559941 & -3.528370 \\
\hline 1 & -1.676704 & 11.051959 & -3.379217 \\
\hline 1 & -1.332369 & 9.448569 & -2.764661 \\
\hline 1 & -1.244778 & 12.064691 & -1.338664 \\
\hline 1 & -0.336958 & 10.591160 & -1.050972 \\
\hline 1 & -4.824280 & 12.543837 & -0.654699 \\
\hline 1 & -3.226124 & 12.754979 & -1.325277 \\
\hline 1 & -1.986959 & 8.648027 & 2.487582 \\
\hline 1 & -2.134451 & 10.350197 & 2.964011 \\
\hline 1 & -4.422182 & 9.454492 & 2.810471 \\
\hline 1 & -4.079914 & 8.731352 & 1.240637 \\
\hline 1 & -4.284957 & 11.718106 & 1.904522 \\
\hline 1 & -5.314109 & 10.783940 & 0.815607 \\
\hline 6 & -1.704487 & 2.227153 & 4.647848 \\
\hline 6 & -1.524466 & 2.083975 & 3.273004 \\
\hline 6 & -1.067140 & 3.155458 & 2.511255 \\
\hline 6 & -0.802208 & 4.387054 & 3.119614 \\
\hline 6 & -1.013784 & 4.533535 & 4.494097 \\
\hline 6 & -1.445863 & 3.453991 & 5.258021 \\
\hline 1 & -2.050473 & 1.387576 & 5.240546 \\
\hline 1 & -1.737303 & 1.135782 & 2.792446 \\
\hline 1 & -0.918203 & 3.039784 & 1.444942 \\
\hline 1 & -0.839352 & 5.500758 & 4.949676 \\
\hline
\end{tabular}




\begin{tabular}{|c|c|c|c|}
\hline 1 & -1.589792 & 3.570842 & 6.326415 \\
\hline 6 & -0.432207 & 5.603281 & 2.319650 \\
\hline 8 & -1.001989 & 6.668984 & 2.565214 \\
\hline 7 & 0.463446 & 5.496957 & 1.284565 \\
\hline 6 & 1.541907 & 4.570795 & 1.116472 \\
\hline 6 & 2.181684 & 4.768557 & -0.104805 \\
\hline 6 & 2.036619 & 3.638881 & 2.035718 \\
\hline 6 & 3.275589 & 3.990472 & -0.457812 \\
\hline 6 & 3.139001 & 2.866541 & 1.671732 \\
\hline 6 & 3.752251 & 3.026838 & 0.428168 \\
\hline 1 & 3.747882 & 4.156211 & -1.419040 \\
\hline 1 & 3.525100 & 2.138295 & 2.376582 \\
\hline 1 & 4.609887 & 2.417640 & 0.164689 \\
\hline 6 & 1.537693 & 5.850119 & -0.970403 \\
\hline 8 & 0.942084 & 5.479497 & -2.020525 \\
\hline 8 & 2.630371 & 6.873171 & -1.175455 \\
\hline 6 & 2.541590 & 7.602922 & -2.384739 \\
\hline 1 & 2.014138 & 7.013223 & -3.139000 \\
\hline 1 & 2.014172 & 8.557622 & -2.241299 \\
\hline 1 & 3.559367 & 7.816685 & -2.726410 \\
\hline 6 & 0.479276 & 6.545106 & 0.235552 \\
\hline 6 & 1.090830 & 7.818052 & 0.764532 \\
\hline 1 & 2.408878 & 6.785992 & 1.914144 \\
\hline 6 & 2.871412 & 8.841646 & 2.136799 \\
\hline 1 & 2.584337 & 9.111929 & 3.160306 \\
\hline 1 & 2.602583 & 9.683534 & 1.494883 \\
\hline 6 & -0.880708 & 6.775108 & -0.432689 \\
\hline 1 & -1.470268 & 7.501100 & 0.130378 \\
\hline 1 & -0.651913 & 7.205584 & -1.411751 \\
\hline 6 & -1.724261 & 5.515282 & -0.663107 \\
\hline 1 & -1.089523 & 4.754199 & -1.120442 \\
\hline 1 & -2.096807 & 5.126232 & 0.291672 \\
\hline 6 & -2.923449 & 5.782751 & -1.579380 \\
\hline 1 & -3.537247 & 6.593352 & -1.159836 \\
\hline 1 & -2.554482 & 6.141814 & -2.547193 \\
\hline 6 & -3.801860 & 4.547171 & -1.797625 \\
\hline 1 & -4.643744 & 4.760646 & -2.463531 \\
\hline 1 & -3.222410 & 3.733865 & -2.244168 \\
\hline 1 & -4.210801 & 4.179624 & -0.850704 \\
\hline 8 & 0.726677 & 8.947817 & 0.362909 \\
\hline 7 & 2.079220 & 7.709791 & 1.677550 \\
\hline 6 & 4.364640 & 8.582797 & 2.086098 \\
\hline 6 & 5.187680 & 9.049249 & 3.114727 \\
\hline 6 & 4.942781 & 7.909556 & 1.003122 \\
\hline
\end{tabular}




$\begin{array}{llll}6 & 6.567404 & 8.861763 & 3.063791 \\ 1 & 4.745571 & 9.560786 & 3.964697 \\ 6 & 6.321859 & 7.716701 & 0.959188 \\ 1 & 4.309024 & 7.529049 & 0.206986 \\ 6 & 7.138519 & 8.193544 & 1.983440 \\ 1 & 7.192625 & 9.228282 & 3.870842 \\ 1 & 6.758741 & 7.188460 & 0.118758 \\ 1 & 8.211277 & 8.039130 & 1.943615 \\ 1 & 1.590364 & 3.507655 & 3.010532 \\ 1 & -0.701529 & 9.574317 & 0.775229\end{array}$

Pathyway 2:

IM1

Sum of electronic and zero-point Energies: -2031.145388 a.u.

\begin{tabular}{crrr} 
Atomic & \multicolumn{3}{c}{ Coordinates (Angstroms) } \\
Number & $\mathrm{X}$ & $\mathrm{Y}$ & $\mathrm{Z}$ \\
\hline- & & & \\
- & -3.055709 & 12.589124 & 2.005543 \\
6 & -1.985108 & 11.856273 & 1.183219 \\
6 & -0.785504 & 11.333846 & 1.988591 \\
6 & -1.104580 & 10.282874 & 3.095433 \\
6 & -2.442672 & 9.577733 & 2.976424 \\
7 & -3.565510 & 10.378338 & 3.091308 \\
6 & -3.447734 & 11.821015 & 3.284104 \\
7 & -2.450107 & 8.300581 & 2.799450 \\
6 & -3.746510 & 7.634059 & 2.699576 \\
6 & -4.818757 & 8.524569 & 2.069898 \\
6 & -4.904397 & 9.836338 & 2.843031 \\
1 & -3.940058 & 12.750685 & 1.378930 \\
1 & -2.698587 & 13.583128 & 2.300329 \\
1 & -2.453177 & 11.012113 & 0.666149 \\
1 & -1.620801 & 12.524692 & 0.396118 \\
1 & -0.256074 & 12.178821 & 2.443432 \\
1 & -0.082996 & 10.869574 & 1.293580 \\
1 & -1.048677 & 10.750971 & 4.084392 \\
1 & -0.344555 & 9.505608 & 3.064085 \\
1 & -4.405095 & 12.184187 & 3.667123 \\
1 & -2.716151 & 12.018995 & 4.070313 \\
1 & -3.610000 & 6.722926 & 2.113461 \\
1 & -4.072348 & 7.321685 & 3.703507 \\
1 & -5.794506 & 8.030483 & 2.063512 \\
1 & -4.547099 & 8.727234 & 1.028774
\end{tabular}




\begin{tabular}{|c|c|c|c|}
\hline 1 & -5.427324 & 9.681389 & 3.797394 \\
\hline 1 & -5.481843 & 10.575772 & 2.277813 \\
\hline 6 & -1.096770 & 1.672750 & 4.630898 \\
\hline U & 0.065040 & 1.882649 & 3.891963 \\
\hline 6 & 0.140139 & 2.935552 & 2.983112 \\
\hline 6 & -0.956805 & 3.782343 & 2.797459 \\
\hline 6 & -2.131829 & 3.547683 & 3.521315 \\
\hline 6 & -2.196647 & 2.509036 & 4.442820 \\
\hline 1 & -1.148565 & 0.857300 & 5.343856 \\
\hline 1 & 0.916753 & 1.223961 & 4.021155 \\
\hline 1 & 1.039496 & 3.085002 & 2.402421 \\
\hline 1 & -2.988365 & 4.185732 & 3.344417 \\
\hline 1 & -3.106956 & 2.346049 & 5.008890 \\
\hline 6 & -0.995873 & 4.882070 & 1.771378 \\
\hline 8 & -2.022515 & 5.097448 & 1.150215 \\
\hline 7 & 0.170556 & 5.615883 & 1.522659 \\
\hline 6 & 1.050277 & 5.902070 & 2.634298 \\
\hline 6 & 2.430972 & 5.619094 & 2.628713 \\
\hline 6 & 0.496157 & 6.570927 & 3.729364 \\
\hline 6 & 3.206642 & 6.015022 & 3.728867 \\
\hline 6 & 1.273953 & 6.918890 & 4.826873 \\
\hline 6 & 2.637370 & 6.639142 & 4.828808 \\
\hline 1 & 4.269274 & 5.811920 & 3.698567 \\
\hline 1 & 0.816667 & 7.430281 & 5.666286 \\
\hline 1 & 3.257052 & 6.918198 & 5.673018 \\
\hline 6 & 3.209980 & 4.937368 & 1.548624 \\
\hline 8 & 4.382572 & 5.155356 & 1.338017 \\
\hline 8 & 2.522870 & 3.990971 & 0.874453 \\
\hline 6 & 3.282029 & 3.252591 & -0.104161 \\
\hline 1 & 2.599974 & 2.497192 & -0.487907 \\
\hline 1 & 3.616337 & 3.911232 & -0.906577 \\
\hline 1 & 4.151079 & 2.787222 & 0.361487 \\
\hline 6 & -0.017653 & 6.701746 & 0.508468 \\
\hline 6 & 1.222609 & 7.635344 & 0.473130 \\
\hline 1 & 2.088966 & 6.473739 & -0.969083 \\
\hline 6 & 3.477362 & 8.020320 & -0.453981 \\
\hline 1 & 4.109555 & 7.821003 & 0.415956 \\
\hline 1 & 3.213591 & 9.080122 & -0.428049 \\
\hline 8 & 1.266885 & 8.654771 & 1.145218 \\
\hline 7 & 2.237567 & 7.246409 & -0.337185 \\
\hline 6 & 4.214209 & 7.669580 & -1.725443 \\
\hline 6 & 3.848372 & 8.251458 & -2.943945 \\
\hline 6 & 5.261287 & 6.742572 & -1.705925 \\
\hline & 4.517724 & 7.919000 & -4.118466 \\
\hline
\end{tabular}




$\begin{array}{lrrr}1 & 3.036841 & 8.971547 & -2.969180 \\ 6 & 5.933068 & 6.409843 & -2.881659 \\ 1 & 5.542938 & 6.281760 & -0.765204 \\ 6 & 5.563446 & 6.997368 & -4.089297 \\ 1 & 4.227396 & 8.382014 & -5.055013 \\ 1 & 6.747948 & 5.694875 & -2.852170 \\ 1 & 6.088458 & 6.741624 & -5.002903 \\ 1 & -0.548071 & 6.863132 & 3.680464 \\ 6 & -0.362657 & 6.177349 & -0.877333 \\ 6 & -1.092486 & 7.004158 & -1.737194 \\ 6 & 0.070226 & 4.935337 & -1.347481 \\ 6 & -1.374682 & 6.604357 & -3.039432 \\ 1 & -1.449557 & 7.963422 & -1.377449 \\ 6 & -0.216472 & 4.531963 & -2.651194 \\ 1 & 0.607146 & 4.277229 & -0.676505 \\ 6 & -0.935238 & 5.365372 & -3.503481 \\ 1 & -1.946884 & 7.256396 & -3.690252 \\ 1 & 0.115324 & 3.559075 & -2.997502 \\ 1 & -1.160417 & 5.049726 & -4.515891 \\ 1 & -0.833238 & 7.339908 & 0.861402\end{array}$

TS1

Sum of electronic and zero-point Energies: -2031.11528 a.u.

\begin{tabular}{|c|c|c|c|}
\hline \multirow{2}{*}{$\begin{array}{l}\text { Atomic } \\
\text { Number }\end{array}$} & \multicolumn{3}{|c|}{ Coordinates (Angstroms) } \\
\hline & $\mathrm{X}$ & $\mathrm{Y}$ & Z \\
\hline 6 & -2.672296 & 12.146073 & 2.282481 \\
\hline 6 & -1.284036 & 11.917826 & 1.665240 \\
\hline 6 & -0.373194 & 10.947523 & 2.432425 \\
\hline 6 & -0.924087 & 9.506142 & 2.638707 \\
\hline 6 & -1.989601 & 9.084122 & 1.657954 \\
\hline 7 & -3.178928 & 9.745311 & 1.714839 \\
\hline 6 & -3.384945 & 10.835447 & 2.674345 \\
\hline 7 & -1.750094 & 8.114720 & 0.821397 \\
\hline 6 & -2.823635 & 7.607160 & -0.036209 \\
\hline 6 & -3.755640 & 8.736941 & -0.459136 \\
\hline 6 & -4.274986 & 9.457467 & 0.778617 \\
\hline 1 & -3.288270 & 12.706751 & 1.571267 \\
\hline 1 & -2.594095 & 12.767460 & 3.181689 \\
\hline 1 & -1.410246 & 11.545692 & 0.642971 \\
\hline 1 & -0.775928 & 12.882233 & 1.568856 \\
\hline 1 & -0.125861 & 11.367532 & 3.412885 \\
\hline 1 & 0.571611 & 10.863295 & 1.890353 \\
\hline
\end{tabular}




\begin{tabular}{|c|c|c|c|}
\hline . & -1.347318 & 9.401604 & 3.642356 \\
\hline | & -0.093905 & 8.805232 & 2.563222 \\
\hline 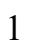 & -4.461812 & 10.996302 & 2.754290 \\
\hline & -3.053819 & 10.509648 & 3.661544 \\
\hline 1 & -2.366897 & 7.129985 & -0.901238 \\
\hline | & -3.360659 & 6.829520 & 0.515809 \\
\hline 1 & -4.595827 & 8.347183 & -1.038825 \\
\hline 1 & -3.212543 & 9.440381 & -1.098173 \\
\hline 1 & -5.033687 & 8.849366 & 1.286627 \\
\hline I & -4.745717 & 10.405685 & 0.503270 \\
\hline 6 & -1.342391 & 2.366678 & 5.910220 \\
\hline 6 & -0.429441 & 2.105503 & 4.891213 \\
\hline 6 & -0.240976 & 3.023766 & 3.860677 \\
\hline 5 & -0.979393 & 4.211924 & 3.834418 \\
\hline 5 & -1.920745 & 4.450582 & 4.842389 \\
\hline 5 & -2.089068 & 3.544077 & 5.882974 \\
\hline ] & -1.478560 & 1.653599 & 6.715819 \\
\hline & 0.140545 & 1.182977 & 4.896169 \\
\hline t & 0.469043 & 2.807119 & 3.073241 \\
\hline 1 & -2.523244 & 5.348991 & 4.788118 \\
\hline | & -2.810202 & 3.749096 & 6.666569 \\
\hline 5 & -0.924821 & 5.201810 & 2.695966 \\
\hline 3 & -1.976207 & 5.672391 & 2.279703 \\
\hline 7 & 0.296476 & 5.489311 & 2.110392 \\
\hline 5 & 1.520201 & 5.292871 & 2.836474 \\
\hline 5 & 2.626122 & 4.620020 & 2.290684 \\
\hline 5 & 1.661701 & 5.897325 & 4.090043 \\
\hline 6 & 3.835590 & 4.587322 & 2.994063 \\
\hline 5 & 2.848252 & 5.813366 & 4.804788 \\
\hline f & 3.948885 & 5.163442 & 4.252748 \\
\hline . & 4.684488 & 4.085020 & 2.544880 \\
\hline 1 & 2.923356 & 6.287604 & 5.776681 \\
\hline 1 & 4.888911 & 5.115322 & 4.789770 \\
\hline 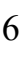 & 2.627877 & 3.884140 & 0.984232 \\
\hline 8 & 3.339345 & 4.151462 & 0.044784 \\
\hline 8 & 1.821362 & 2.806655 & 1.016582 \\
\hline 6 & 1.850758 & 1.967982 & -0.158043 \\
\hline 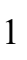 & 1.164963 & 1.151225 & 0.055202 \\
\hline 1 & 1.519767 & 2.528763 & -1.030619 \\
\hline & 2.861288 & 1.591129 & -0.321681 \\
\hline & 0.331237 & 6.300059 & 0.866338 \\
\hline & 1.505440 & 7.233326 & 0.894582 \\
\hline & 2.372840 & 6.388843 & -0.749386 \\
\hline & 3.599399 & 8.008248 & -0.168812 \\
\hline
\end{tabular}




$\begin{array}{lrrr}1 & 4.470205 & 7.360944 & -0.019773 \\ 1 & 3.534191 & 8.671372 & 0.696109 \\ 8 & 1.666521 & 8.028961 & 1.828581 \\ 7 & 2.387934 & 7.202430 & -0.151762 \\ 6 & 3.774507 & 8.808973 & -1.445159 \\ 6 & 2.754403 & 9.647780 & -1.909086 \\ 6 & 4.966719 & 8.746400 & -2.168797 \\ 6 & 2.925590 & 10.405412 & -3.063248 \\ 1 & 1.821494 & 9.700175 & -1.357957 \\ 6 & 5.143380 & 9.505544 & -3.325548 \\ 1 & 5.763706 & 8.094068 & -1.825819 \\ 6 & 4.122945 & 10.337461 & -3.776410 \\ 1 & 2.125575 & 11.051703 & -3.408322 \\ 1 & 6.075780 & 9.440893 & -3.875773 \\ 1 & 4.255895 & 10.926442 & -4.677072 \\ 1 & 0.828302 & 6.461213 & 4.487426 \\ 6 & -0.049299 & 5.573974 & -0.379503 \\ 6 & 0.012143 & 6.210014 & -1.642980 \\ 6 & -0.654439 & 4.302063 & -0.356339 \\ 6 & -0.469367 & 5.601744 & -2.796978 \\ 1 & 0.434605 & 7.203320 & -1.721194 \\ 6 & -1.149632 & 3.702276 & -1.511160 \\ 1 & -0.747242 & 3.773790 & 0.581301 \\ 6 & -1.058905 & 4.339274 & -2.746149 \\ 1 & -0.388018 & 6.126571 & -3.743444 \\ 1 & -1.615185 & 2.724255 & -1.439098 \\ 1 & -1.441959 & 3.868480 & -3.644314 \\ 1 & -0.776842 & 7.332454 & 0.926656\end{array}$

IM2

Sum of electronic and zero-point Energies: -2031.126087 a.u.

\begin{tabular}{crrr} 
Atomic & \multicolumn{3}{c}{ Coordinates (Angstroms) } \\
Number & $\mathrm{X}$ & $\mathrm{Y}$ & $\mathrm{Z}$ \\
\hline$-\mathrm{y}$ & -1.234808 & 11.823578 & 0.840282 \\
6 & -0.059366 & 10.884905 & 1.154790 \\
6 & -0.134122 & 10.218338 & 2.534884 \\
6 & -1.272569 & 9.166596 & 2.733360 \\
6 & -2.131855 & 8.843519 & 1.535048 \\
7 & -2.743686 & 9.826523 & 0.857835 \\
6 & -2.582035 & 11.228651 & 1.271020 \\
7 & -2.292697 & 7.582463 & 1.208052 \\
6 & -3.141041 & 7.133057 & 0.104763
\end{tabular}




\begin{tabular}{|c|c|c|c|}
\hline 6 & -3.172842 & 8.211400 & -0.970332 \\
\hline 5 & -3.542729 & 9.555602 & -0.351300 \\
\hline 1 & -1.248699 & 12.055902 & -0.229685 \\
\hline & -1.113729 & 12.777507 & 1.365386 \\
\hline & 0.010202 & 10.108183 & 0.387352 \\
\hline & 0.875404 & 11.446668 & 1.082669 \\
\hline & -0.224781 & 10.988805 & 3.307849 \\
\hline & 0.801696 & 9.686581 & 2.705345 \\
\hline 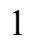 & -1.956915 & 9.486513 & 3.527811 \\
\hline & -0.824766 & 8.229366 & 3.060617 \\
\hline & -3.417318 & 11.788464 & 0.846363 \\
\hline 1 & -2.690835 & 11.285117 & 2.355764 \\
\hline 1 & -2.722676 & 6.203048 & -0.277232 \\
\hline & -4.147417 & 6.924350 & 0.486177 \\
\hline 1 & -3.894393 & 7.953575 & -1.747706 \\
\hline 1 & -2.187911 & 8.270674 & -1.439965 \\
\hline 1 & -4.606272 & 9.584664 & -0.086041 \\
\hline 1 & -3.358044 & 10.367461 & -1.058804 \\
\hline & -1.140358 & 1.987998 & 6.215898 \\
\hline & -0.268710 & 1.721360 & 5.161579 \\
\hline & -0.020335 & 2.688995 & 4.192623 \\
\hline & -0.652944 & 3.936701 & 4.265945 \\
\hline & -1.552690 & 4.185239 & 5.309318 \\
\hline & -1.783056 & 3.223627 & 6.286503 \\
\hline & -1.325215 & 1.234088 & 6.973096 \\
\hline & 0.218942 & 0.755573 & 5.090772 \\
\hline & 0.652472 & 2.471673 & 3.372429 \\
\hline$\frac{1}{1}$ & -2.069132 & 5.136736 & 5.334539 \\
\hline & -2.470566 & 3.432899 & 7.098627 \\
\hline & -0.499323 & 4.980783 & 3.201182 \\
\hline & -1.501242 & 5.649488 & 2.893764 \\
\hline & 0.688976 & 5.088882 & 2.528126 \\
\hline & 1.935466 & 4.822278 & 3.184592 \\
\hline & 3.005326 & 4.236926 & 2.489736 \\
\hline & 2.147532 & 5.279032 & 4.488193 \\
\hline f & 4.247141 & 4.105671 & 3.115353 \\
\hline & 3.373032 & 5.099938 & 5.117946 \\
\hline & 4.432459 & 4.512645 & 4.432287 \\
\hline & 5.067383 & 3.674605 & 2.553392 \\
\hline & 3.510055 & 5.459422 & 6.131605 \\
\hline & 5.399447 & 4.394644 & 4.907246 \\
\hline & 2.916309 & 3.754113 & 1.073214 \\
\hline & 3.637387 & 4.131389 & 0.177603 \\
\hline & 2.035114 & 2.743366 & 0.942885 \\
\hline
\end{tabular}




\begin{tabular}{|c|c|c|}
\hline 1.948782 & 2.162198 & -0.374450 \\
\hline 1.201133 & 1.376970 & -0.294904 \\
\hline 1.633993 & 2.910808 & -1.099782 \\
\hline 2.916714 & 1.750313 & -0.664890 \\
\hline 0.778032 & 5.723477 & 1.232286 \\
\hline 1.582911 & 6.926536 & 1.262149 \\
\hline 2.497196 & 6.481953 & -0.493419 \\
\hline 3.333438 & 8.270871 & 0.169045 \\
\hline 4.297536 & 7.796107 & 0.393835 \\
\hline 3.100453 & 8.903727 & 1.028418 \\
\hline 1.713666 & 7.622073 & 2.287319 \\
\hline 2.274286 & 7.275848 & 0.093633 \\
\hline 3.478739 & 9.115244 & -1.083464 \\
\hline 2.385651 & 9.426422 & -1.897553 \\
\hline 4.729804 & 9.638501 & -1.426321 \\
\hline 2.536577 & 10.243766 & -3.015929 \\
\hline 1.416500 & 9.009051 & -1.651185 \\
\hline 4.884502 & 10.459469 & -2.540728 \\
\hline 5.593230 & 9.394299 & -0.814920 \\
\hline 3.786408 & 10.766435 & -3.341632 \\
\hline 1.677136 & 10.467976 & -3.639339 \\
\hline 5.864989 & 10.851051 & -2.789507 \\
\hline 3.905584 & 11.398184 & -4.214868 \\
\hline 1.350788 & 5.807128 & 4.994076 \\
\hline 0.013249 & 5.192058 & 0.132952 \\
\hline-0.039841 & 5.805650 & -1.155603 \\
\hline-0.798759 & 4.028227 & 0.270923 \\
\hline-0.804612 & 5.287818 & -2.193490 \\
\hline 0.534891 & 6.701421 & -1.339133 \\
\hline-1.566824 & 3.527681 & -0.771405 \\
\hline-0.816164 & 3.498969 & 1.212740 \\
\hline-1.586239 & 4.142945 & -2.024943 \\
\hline-0.785640 & 5.792666 & -3.155366 \\
\hline-2.160473 & 2.634725 & -0.598487 \\
\hline-2.178914 & 3.741556 & -2.838605 \\
\hline-1.822639 & 6.851567 & 1.786514 \\
\hline
\end{tabular}

TS2

Sum of electronic and zero-point Energies: -2031.114333 a.u.

\begin{tabular}{cccc} 
Atomic & \multicolumn{3}{c}{ Coordinates (Angstroms) } \\
Number & $\mathrm{X}$ & $\mathrm{Y}$ & $\mathrm{Z}$ \\
$-\mathrm{-} 6$ & -0.424826 & 11.873455 & 1.899036
\end{tabular}




\begin{tabular}{|c|c|c|c|}
\hline 6 & 0.456370 & 10.693764 & 2.334931 \\
\hline 6 & -0.150470 & 9.744207 & 3.376996 \\
\hline 6 & -1.487026 & 9.050211 & 2.976904 \\
\hline 6 & -1.797452 & 9.022944 & 1.503431 \\
\hline 7 & -1.958530 & 10.178446 & 0.844602 \\
\hline 6 & -1.868550 & 11.460377 & 1.560596 \\
\hline 7 & -1.952104 & 7.862250 & 0.910269 \\
\hline 6 & -2.272955 & 7.729742 & -0.510425 \\
\hline 6 & -1.686734 & 8.915803 & -1.268305 \\
\hline 6 & -2.138964 & 10.219451 & -0.619341 \\
\hline 1 & 0.035456 & 12.355699 & 1.030690 \\
\hline 1 & -0.477543 & 12.634129 & 2.685952 \\
\hline 1 & 0.730644 & 10.093768 & 1.464769 \\
\hline 1 & 1.396241 & 11.087034 & 2.733881 \\
\hline 1 & -0.309112 & 10.266910 & 4.326043 \\
\hline 1 & 0.583259 & 8.957684 & 3.550371 \\
\hline 1 & -2.333783 & 9.543722 & 3.466311 \\
\hline 1 & -1.486134 & 8.018682 & 3.323627 \\
\hline 1 & -2.352858 & 12.210734 & 0.933317 \\
\hline 1 & -2.465997 & 11.389995 & 2.471212 \\
\hline 1 & -1.854391 & 6.786014 & -0.857306 \\
\hline 1 & -3.361404 & 7.683053 & -0.631639 \\
\hline 1 & -2.005879 & 8.899128 & -2.311948 \\
\hline 1 & -0.596459 & 8.852831 & -1.253242 \\
\hline 1 & -3.193751 & 10.424188 & -0.837931 \\
\hline 1 & -1.550257 & 11.055896 & -1.002147 \\
\hline 6 & -1.499890 & 2.626221 & 6.379270 \\
\hline 6 & -0.577311 & 2.156749 & 5.445584 \\
\hline 6 & -0.210819 & 2.948720 & 4.361894 \\
\hline 6 & -0.777312 & 4.219108 & 4.196925 \\
\hline 6 & -1.727902 & 4.669874 & 5.118691 \\
\hline 6 & -2.075596 & 3.885195 & 6.213213 \\
\hline 1 & -1.776593 & 2.009560 & 7.227239 \\
\hline 1 & -0.141447 & 1.170799 & 5.559877 \\
\hline 1 & 0.504742 & 2.580541 & 3.636473 \\
\hline 1 & -2.195783 & 5.633543 & 4.959349 \\
\hline 1 & -2.802631 & 4.249247 & 6.930692 \\
\hline 6 & -0.496367 & 5.054379 & 2.980588 \\
\hline 8 & -1.439188 & 5.688907 & 2.480136 \\
\hline 7 & 0.744185 & 4.985318 & 2.419115 \\
\hline 6 & 1.954941 & 4.800414 & 3.158349 \\
\hline 6 & 2.998728 & 4.327108 & 2.370870 \\
\hline 6 & 2.176572 & 5.173181 & 4.483944 \\
\hline 6 & 4.274609 & 4.197380 & 2.900782 \\
\hline
\end{tabular}




\begin{tabular}{|c|c|c|c|}
\hline 6 & 3.455245 & 5.018244 & 5.017983 \\
\hline 6 & 4.503653 & 4.534486 & 4.234843 \\
\hline 1 & 5.075430 & 3.845691 & 2.260269 \\
\hline 1 & 3.636318 & 5.303872 & 6.048416 \\
\hline 1 & 5.497287 & 4.439047 & 4.658607 \\
\hline 6 & 2.629831 & 4.017508 & 0.929150 \\
\hline 8 & 3.409791 & 4.302132 & -0.021786 \\
\hline 8 & 2.044882 & 2.724599 & 0.945105 \\
\hline 6 & 2.167682 & 1.985158 & -0.270071 \\
\hline 1 & 1.801831 & 0.983281 & -0.040547 \\
\hline 1 & 1.565724 & 2.416029 & -1.073854 \\
\hline 1 & 3.208889 & 1.943613 & -0.595444 \\
\hline 6 & 1.050726 & 5.200674 & 0.999844 \\
\hline 6 & 1.520756 & 6.639978 & 0.800769 \\
\hline 1 & 3.014406 & 5.918171 & -0.334104 \\
\hline 6 & 3.180476 & 8.070797 & -0.283404 \\
\hline 1 & 4.227403 & 7.851360 & -0.517159 \\
\hline 1 & 3.159690 & 8.612013 & 0.665656 \\
\hline 8 & 1.060966 & 7.583303 & 1.454888 \\
\hline 7 & 2.500824 & 6.795424 & -0.123806 \\
\hline 6 & 2.611075 & 8.963647 & -1.375659 \\
\hline 6 & 2.242667 & 8.439916 & -2.619472 \\
\hline 6 & 2.493590 & 10.341393 & -1.173637 \\
\hline 6 & 1.766634 & 9.270455 & -3.631161 \\
\hline 1 & 2.335456 & 7.373800 & -2.793353 \\
\hline 6 & 2.020139 & 11.178014 & -2.184678 \\
\hline 1 & 2.780382 & 10.763306 & -0.215564 \\
\hline 6 & 1.652222 & 10.644155 & -3.417798 \\
\hline 1 & 1.492657 & 8.846096 & -4.591054 \\
\hline 1 & 1.945772 & 12.246415 & -2.010635 \\
\hline 1 & 1.288736 & 11.291637 & -4.208144 \\
\hline 1 & 1.379889 & 5.584861 & 5.089561 \\
\hline 6 & 0.093951 & 4.664873 & 0.002871 \\
\hline 6 & 0.117802 & 5.111641 & -1.334518 \\
\hline 6 & -0.792823 & 3.610935 & 0.300731 \\
\hline 6 & -0.695396 & 4.541270 & -2.310493 \\
\hline 1 & 0.800823 & 5.900702 & -1.615780 \\
\hline 6 & -1.607350 & 3.047973 & -0.674509 \\
\hline 1 & -0.836363 & 3.209366 & 1.302366 \\
\hline 6 & -1.570166 & 3.505405 & -1.991019 \\
\hline 1 & -0.635105 & 4.907429 & -3.330682 \\
\hline 1 & -2.274320 & 2.236943 & -0.401060 \\
\hline 1 & -2.203526 & 3.059863 & -2.749913 \\
\hline 1 & -1.682936 & 7.011438 & 1.441318 \\
\hline
\end{tabular}




\section{IM3}

Sum of electronic and zero-point Energies: -2031.115292 a.u.

\begin{tabular}{|c|c|c|c|}
\hline \multirow{2}{*}{$\begin{array}{l}\text { Atomic } \\
\text { Number }\end{array}$} & \multicolumn{3}{|c|}{ Coordinates (Angstroms) } \\
\hline & $\mathrm{X}$ & $\mathrm{Y}$ & $\mathrm{Z}$ \\
\hline 6 & -4.358292 & 11.156746 & -2.274866 \\
\hline 6 & -3.388539 & 9.999548 & -2.559449 \\
\hline 6 & -1.895497 & 10.360846 & -2.559839 \\
\hline 6 & -1.336834 & 10.978839 & -1.241225 \\
\hline 6 & -2.170781 & 10.708228 & -0.014859 \\
\hline 7 & -3.416316 & 11.199121 & 0.059488 \\
\hline 6 & -3.963494 & 12.000440 & -1.046969 \\
\hline 7 & -1.643523 & 10.000863 & 0.956068 \\
\hline 6 & -2.362496 & 9.623686 & 2.176022 \\
\hline 6 & -3.858846 & 9.586485 & 1.884979 \\
\hline 6 & -4.296254 & 10.878756 & 1.199569 \\
\hline 1 & -5.364065 & 10.747474 & -2.136931 \\
\hline 1 & -4.414200 & 11.832303 & -3.135070 \\
\hline 1 & -3.556028 & 9.202960 & -1.827960 \\
\hline 1 & -3.639839 & 9.559941 & -3.528370 \\
\hline 1 & -1.676704 & 11.051959 & -3.379217 \\
\hline 1 & -1.332369 & 9.448569 & -2.764661 \\
\hline 1 & -1.244778 & 12.064691 & -1.338664 \\
\hline 1 & -0.336958 & 10.591160 & -1.050972 \\
\hline 1 & -4.824280 & 12.543837 & -0.654699 \\
\hline 1 & -3.226124 & 12.754979 & -1.325277 \\
\hline 1 & -1.986959 & 8.648027 & 2.487582 \\
\hline 1 & -2.134451 & 10.350197 & 2.964011 \\
\hline 1 & -4.422182 & 9.454492 & 2.810471 \\
\hline 1 & -4.079914 & 8.731352 & 1.240637 \\
\hline 1 & -4.284957 & 11.718106 & 1.904522 \\
\hline 1 & -5.314109 & 10.783940 & 0.815607 \\
\hline 6 & -1.704487 & 2.227153 & 4.647848 \\
\hline 6 & -1.524466 & 2.083975 & 3.273004 \\
\hline 6 & -1.067140 & 3.155458 & 2.511255 \\
\hline 6 & -0.802208 & 4.387054 & 3.119614 \\
\hline 6 & -1.013784 & 4.533535 & 4.494097 \\
\hline 6 & -1.445863 & 3.453991 & 5.258021 \\
\hline 1 & -2.050473 & 1.387576 & 5.240546 \\
\hline 1 & -1.737303 & 1.135782 & 2.792446 \\
\hline 1 & -0.918203 & 3.039784 & 1.444942 \\
\hline 1 & -0.839352 & 5.500758 & 4.949676 \\
\hline
\end{tabular}




\begin{tabular}{|c|c|c|c|}
\hline 1 & -1.589792 & 3.570842 & 6.326415 \\
\hline 6 & -0.432207 & 5.603281 & 2.319650 \\
\hline 8 & -1.001989 & 6.668984 & 2.565214 \\
\hline 7 & 0.463446 & 5.496957 & 1.284565 \\
\hline 6 & 1.541907 & 4.570795 & 1.116472 \\
\hline 6 & 2.181684 & 4.768557 & -0.104805 \\
\hline 6 & 2.036619 & 3.638881 & 2.035718 \\
\hline 6 & 3.275589 & 3.990472 & -0.457812 \\
\hline 6 & 3.139001 & 2.866541 & 1.671732 \\
\hline 6 & 3.752251 & 3.026838 & 0.428168 \\
\hline 1 & 3.747882 & 4.156211 & -1.419040 \\
\hline 1 & 3.525100 & 2.138295 & 2.376582 \\
\hline 1 & 4.609887 & 2.417640 & 0.164689 \\
\hline 6 & 1.537693 & 5.850119 & -0.970403 \\
\hline 8 & 0.942084 & 5.479497 & -2.020525 \\
\hline 8 & 2.630371 & 6.873171 & -1.175455 \\
\hline 6 & 2.541590 & 7.602922 & -2.384739 \\
\hline 1 & 2.014138 & 7.013223 & -3.139000 \\
\hline 1 & 2.014172 & 8.557622 & -2.241299 \\
\hline 1 & 3.559367 & 7.816685 & -2.726410 \\
\hline 6 & 0.479276 & 6.545106 & 0.235552 \\
\hline 6 & 1.090830 & 7.818052 & 0.764532 \\
\hline 1 & 2.408878 & 6.785992 & 1.914144 \\
\hline 6 & 2.871412 & 8.841646 & 2.136799 \\
\hline 1 & 2.584337 & 9.111929 & 3.160306 \\
\hline 1 & 2.602583 & 9.683534 & 1.494883 \\
\hline 6 & -0.880708 & 6.775108 & -0.432689 \\
\hline 1 & -1.470268 & 7.501100 & 0.130378 \\
\hline 1 & -0.651913 & 7.205584 & -1.411751 \\
\hline 6 & -1.724261 & 5.515282 & -0.663107 \\
\hline 1 & -1.089523 & 4.754199 & -1.120442 \\
\hline 1 & -2.096807 & 5.126232 & 0.291672 \\
\hline 6 & -2.923449 & 5.782751 & -1.579380 \\
\hline 1 & -3.537247 & 6.593352 & -1.159836 \\
\hline 1 & -2.554482 & 6.141814 & -2.547193 \\
\hline 6 & -3.801860 & 4.547171 & -1.797625 \\
\hline 1 & -4.643744 & 4.760646 & -2.463531 \\
\hline 1 & -3.222410 & 3.733865 & -2.244168 \\
\hline 1 & -4.210801 & 4.179624 & -0.850704 \\
\hline 8 & 0.726677 & 8.947817 & 0.362909 \\
\hline 7 & 2.079220 & 7.709791 & 1.677550 \\
\hline 6 & 4.364640 & 8.582797 & 2.086098 \\
\hline 6 & 5.187680 & 9.049249 & 3.114727 \\
\hline 6 & 4.942781 & 7.909556 & 1.003122 \\
\hline
\end{tabular}




$\begin{array}{lrrr}6 & 6.567404 & 8.861763 & 3.063791 \\ 1 & 4.745571 & 9.560786 & 3.964697 \\ 6 & 6.321859 & 7.716701 & 0.959188 \\ 1 & 4.309024 & 7.529049 & 0.206986 \\ 6 & 7.138519 & 8.193544 & 1.983440 \\ 1 & 7.192625 & 9.228282 & 3.870842 \\ 1 & 6.758741 & 7.188460 & 0.118758 \\ 1 & 8.211277 & 8.039130 & 1.943615 \\ 1 & 1.590364 & 3.507655 & 3.010532 \\ 1 & -0.701529 & 9.574317 & 0.775229\end{array}$




\section{X-Ray Analysis of $6 \mathrm{~h}$ and 10e}

Single crystals of $\mathbf{6 h}$ were grown from methanol/dichloromethane at room temperature by slow evaporation. Crystallographic studies were undertaken on single crystal mounted on Broker Apex II diffractometer with Apex II CCD Detector, $\mathrm{Cu}$-Microsource and Oxford Cryosystem 700 Plus low temperature Device.

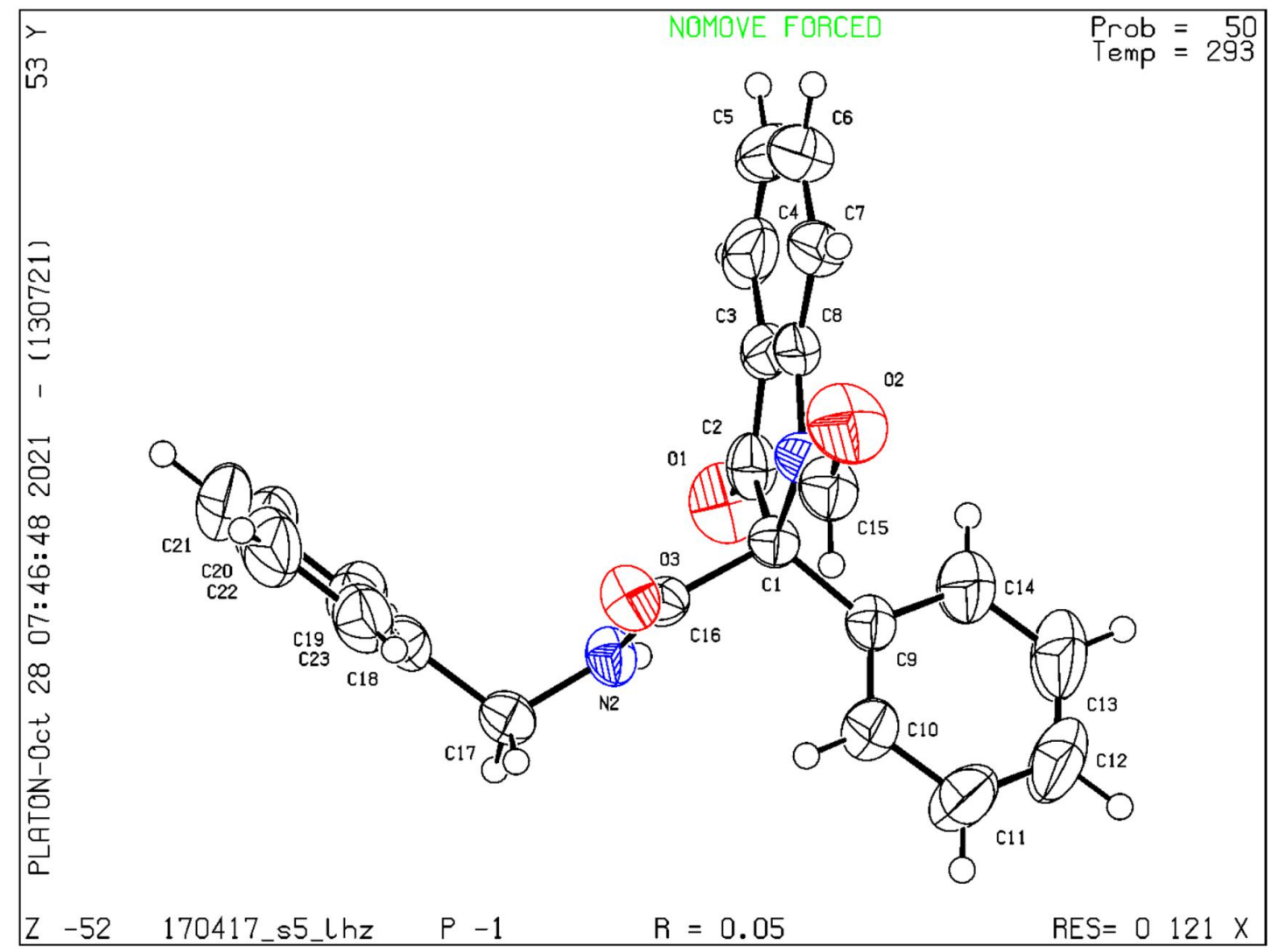

Figure S2. Solid-state structure of $\mathbf{6 h}$. C: black, N: blue, O: red. 
Table S1. Crystal data and structure refinement for $\mathbf{6 h}$.

\begin{tabular}{|c|c|}
\hline Identification code & $6 \mathrm{~h}$ \\
\hline Empirical formula & $\mathrm{C}_{23} \mathrm{H}_{18} \mathrm{~N}_{2} \mathrm{O}_{3}$ \\
\hline Formula weight & 370.39 \\
\hline Temperature/K & 293.15 \\
\hline Crystal system & triclinic \\
\hline Space group & $\mathrm{P}-1$ \\
\hline $\mathrm{a} / \AA$ & $8.1633(5)$ \\
\hline $\mathrm{b} / \AA$ & $10.504(2)$ \\
\hline $\mathrm{c} / \AA$ & $11.8734(9)$ \\
\hline$\alpha /{ }^{\circ}$ & $73.131(13)$ \\
\hline$\beta /{ }^{\circ}$ & $89.827(6)$ \\
\hline$\gamma /{ }^{\circ}$ & $77.915(11)$ \\
\hline Volume $/ \AA^{3}$ & $950.9(2)$ \\
\hline Z & 2 \\
\hline$\rho_{\text {calc }} \mathrm{g} / \mathrm{cm}^{3}$ & 1.294 \\
\hline$\mu / \mathrm{mm}^{-1}$ & 0.087 \\
\hline $\mathrm{F}(000)$ & 388.0 \\
\hline Crystal size $/ \mathrm{mm}^{3}$ & $0.35 \times 0.25 \times 0.2$ \\
\hline Radiation & $\operatorname{MoK} \alpha(\lambda=0.71073)$ \\
\hline $2 \Theta$ range for data collection $/^{\circ}$ & 6.066 to 52.736 \\
\hline Index ranges & $\begin{array}{l}-10 \leq \mathrm{h} \leq 10,-13 \leq \mathrm{k} \leq 13,-14 \leq \\
1 \leq 14\end{array}$ \\
\hline Reflections collected & 5785 \\
\hline Independent reflections & $5785\left[\mathrm{R}_{\mathrm{int}}=?, \mathrm{R}_{\text {sigma }}=0.0582\right]$ \\
\hline Data/restraints/parameters & $5785 / 0 / 254$ \\
\hline Goodness-of-fit on $\mathrm{F}^{2}$ & 0.889 \\
\hline Final $\mathrm{R}$ indexes $[\mathrm{I}>=2 \sigma(\mathrm{I})]$ & $\mathrm{R}_{1}=0.0455, \mathrm{wR}_{2}=0.0958$ \\
\hline Final $\mathrm{R}$ indexes [all data] & $\mathrm{R}_{1}=0.0863, \mathrm{wR}_{2}=0.1042$ \\
\hline Largest diff. peak/hole / e $\AA^{-3}$ & $0.14 /-0.16$ \\
\hline
\end{tabular}


Single crystals of 10e were grown from methanol/dichloromethane at room temperature by slow evaporation. Crystallographic studies were undertaken on single crystal mounted on Broker Apex II diffractometer with Apex II CCD Detector, $\mathrm{Cu}$-Microsource and Oxford Cryosystem 700 Plus low temperature Device.

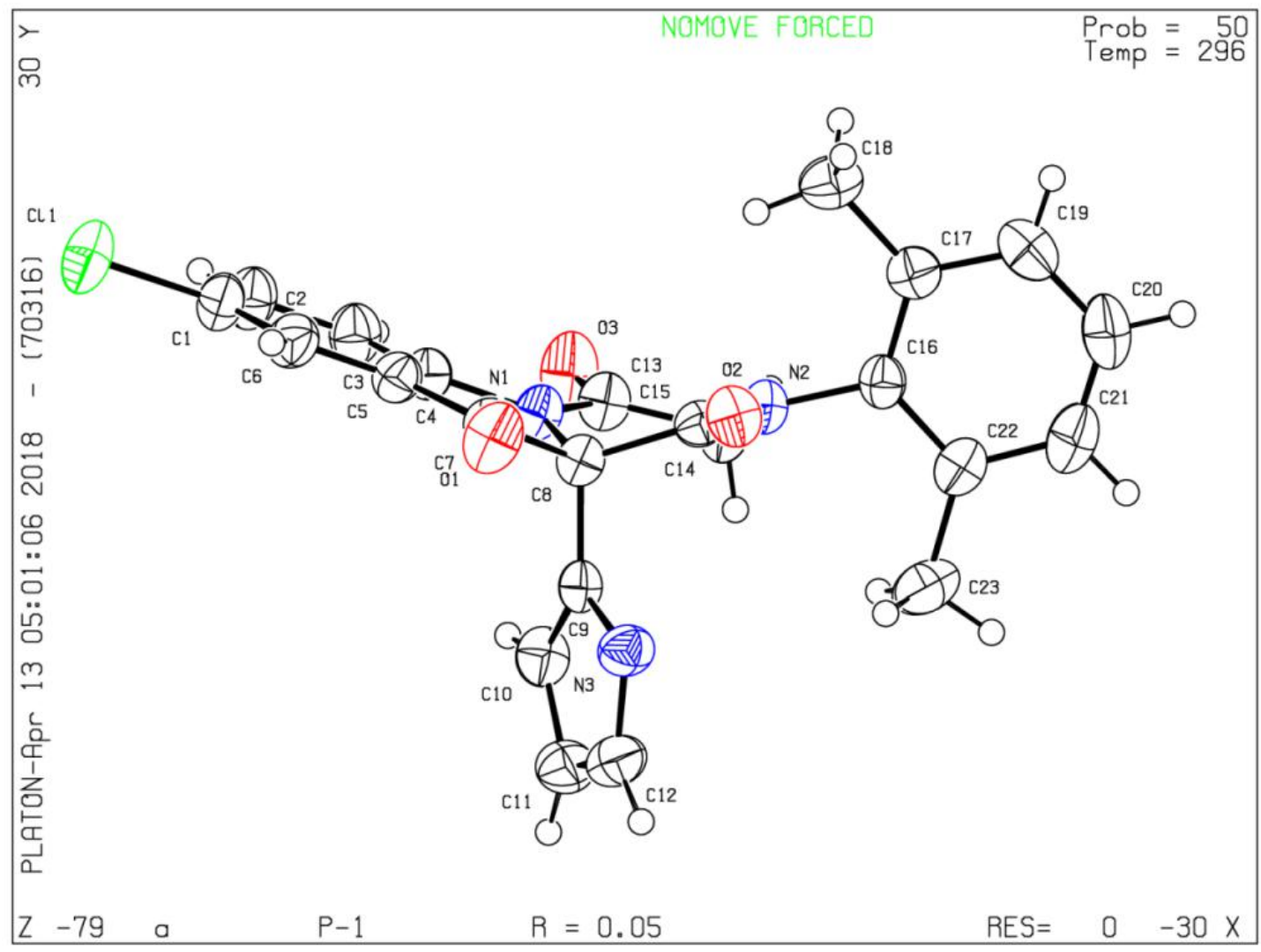

Figure S3. Solid-state structure of 10e. C: black, N: blue, O: red, Cl:green. 
Table S2. Crystal data and structure refinement for 10e.

\begin{tabular}{|c|c|}
\hline Identification code & $10 \mathrm{e}$ \\
\hline Empirical formula & $\mathrm{C}_{23} \mathrm{H}_{17} \mathrm{ClN}_{3} \mathrm{O}_{3}$ \\
\hline Formula weight & 418.85 \\
\hline Temperature/K & $296(2)$ \\
\hline Crystal system & triclinic \\
\hline Space group & P-1 \\
\hline $\mathrm{a} / \AA$ & $8.0880(18)$ \\
\hline $\mathrm{b} / \AA$ & $10.323(2)$ \\
\hline $\mathrm{c} / \AA ̊$ & 12.494(3) \\
\hline$\alpha /^{\circ}$ & $81.837(4)$ \\
\hline$\beta /{ }^{\circ}$ & $89.052(4)$ \\
\hline$\gamma /{ }^{\circ}$ & $69.666(4)$ \\
\hline Volume $/ \AA^{3}$ & $967.9(4)$ \\
\hline Z & 2 \\
\hline$\rho_{\text {calc }} \mathrm{g} / \mathrm{cm}^{3}$ & 1.438 \\
\hline$\mu / \mathrm{mm}^{-1}$ & 0.229 \\
\hline $\mathrm{F}(000)$ & 434.0 \\
\hline Crystal size $/ \mathrm{mm}^{3}$ & $0.29 \times 0.25 \times 0.21$ \\
\hline $2 \Theta$ range for data collection ${ }^{\circ}$ & 1.65 to 25.00 \\
\hline Index ranges & $-5 \leq \mathrm{h} \leq 9,-12 \leq \mathrm{k} \leq 12,-14 \leq 1 \leq 14$ \\
\hline Reflections collected & 4887 \\
\hline Independent reflections & $3369\left[\mathrm{R}_{\mathrm{int}}=0.0214\right]$ \\
\hline Data/restraints/parameters & $3369 / 0 / 271$ \\
\hline Goodness-of-fit on $\mathrm{F}^{2}$ & 1.025 \\
\hline Final $R$ indexes $[I>=2 \sigma(I)]$ & $\mathrm{R}_{1}=0.0481, \mathrm{wR}_{2}=0.1064$ \\
\hline Final $R$ indexes [all data] & $\mathrm{R}_{1}=0.0627, \mathrm{wR}_{2}=0.1096$ \\
\hline Largest diff. peak/hole / e $\AA^{-3}$ & $0.499 /-0.300$ \\
\hline
\end{tabular}




\section{NMR spectra of Compound 6}

${ }^{1} \mathrm{H}$ NMR of Compound 6a (400 MHz, DMSO-d6)

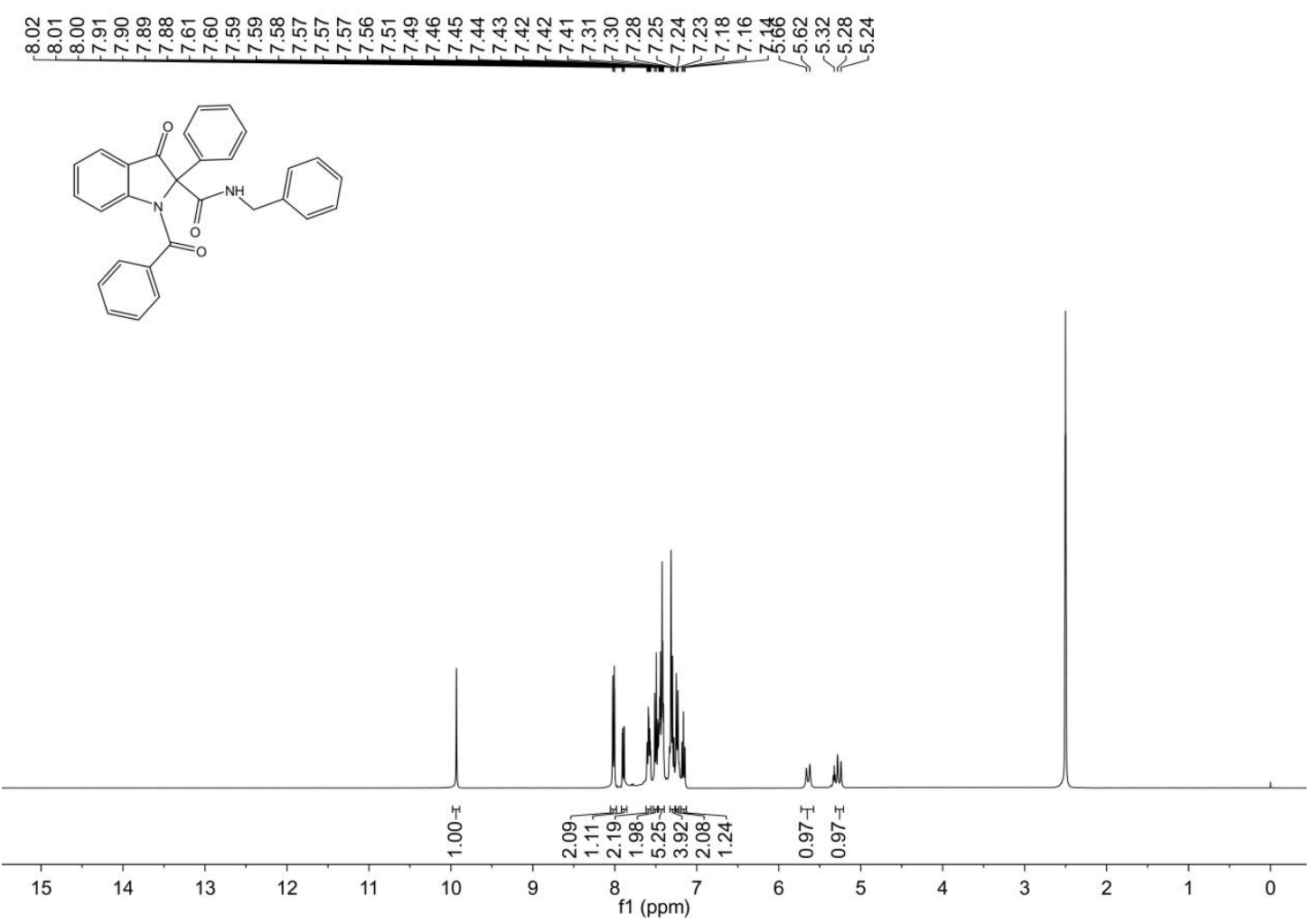

${ }^{13} \mathrm{C}\left\{{ }^{1} \mathrm{H}\right\}$ NMR of Compound 6a $(100 \mathrm{MHz}, \mathrm{DMSO}-d 6)$

¿

蕰

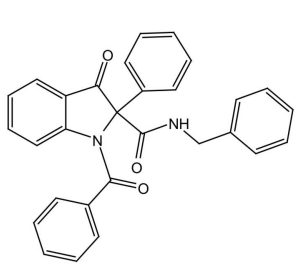

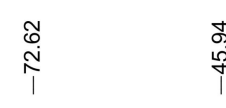

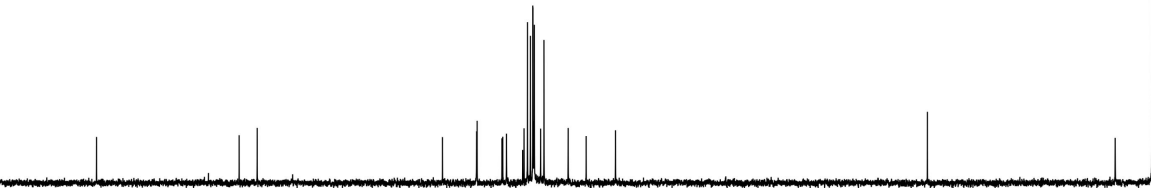

$\begin{array}{lllllllllllllllllllll}200 & 190 & 180 & 170 & 160 & 150 & 140 & 130 & 120 & 110 & 100 & 90 & 80 & 70 & 60 & 50 & 40 & 30 & 20 & 10 & 0\end{array}$ 
${ }^{1} \mathrm{H}$ NMR of Compound 6b (400 MHz, DMSO-d6)

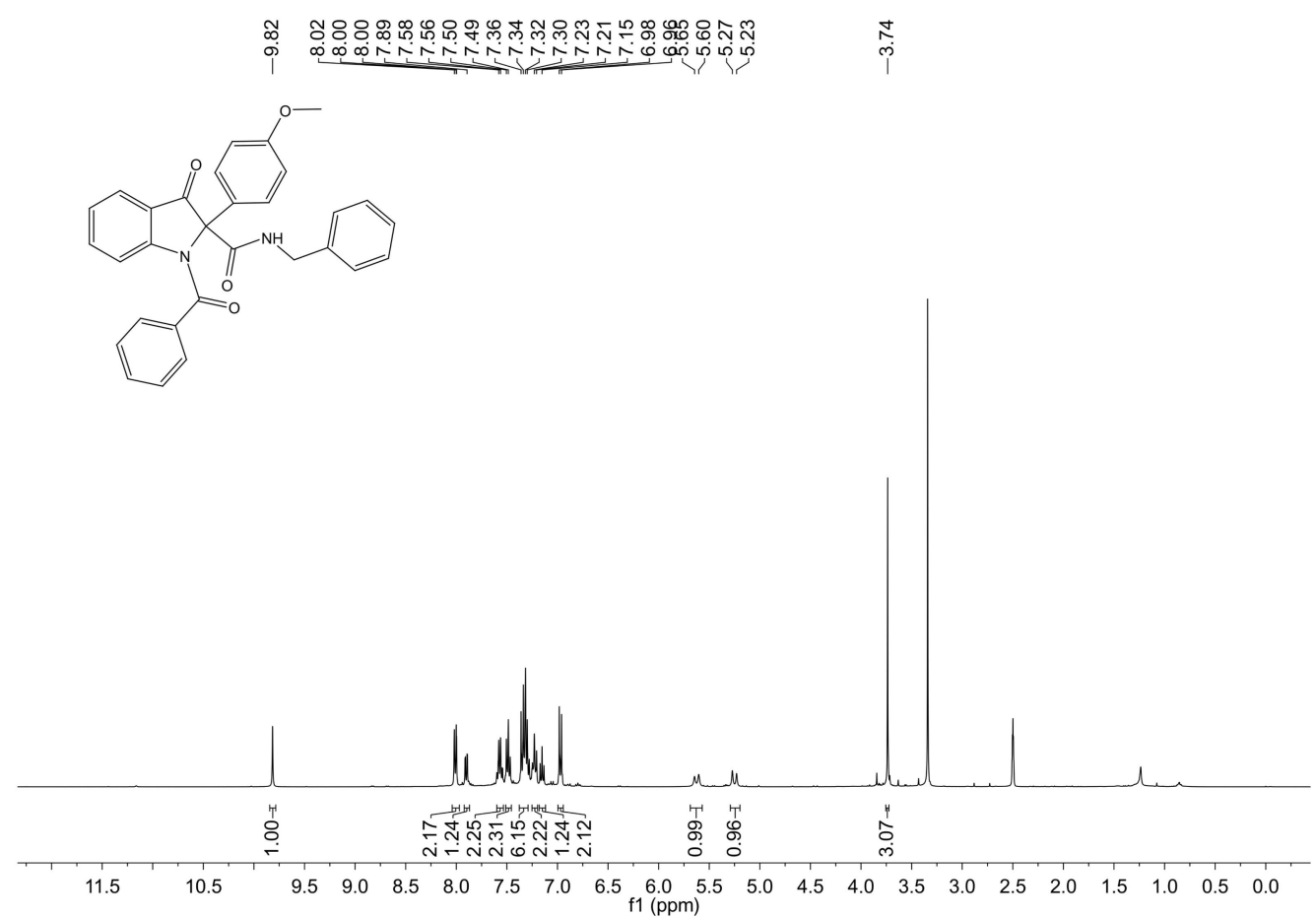

${ }^{13} \mathrm{C}\left\{{ }^{1} \mathrm{H}\right\}$ NMR of Compound $6 \mathbf{b}(100 \mathrm{MHz}, \mathrm{DMSO}-d 6)$

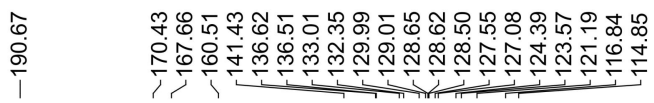
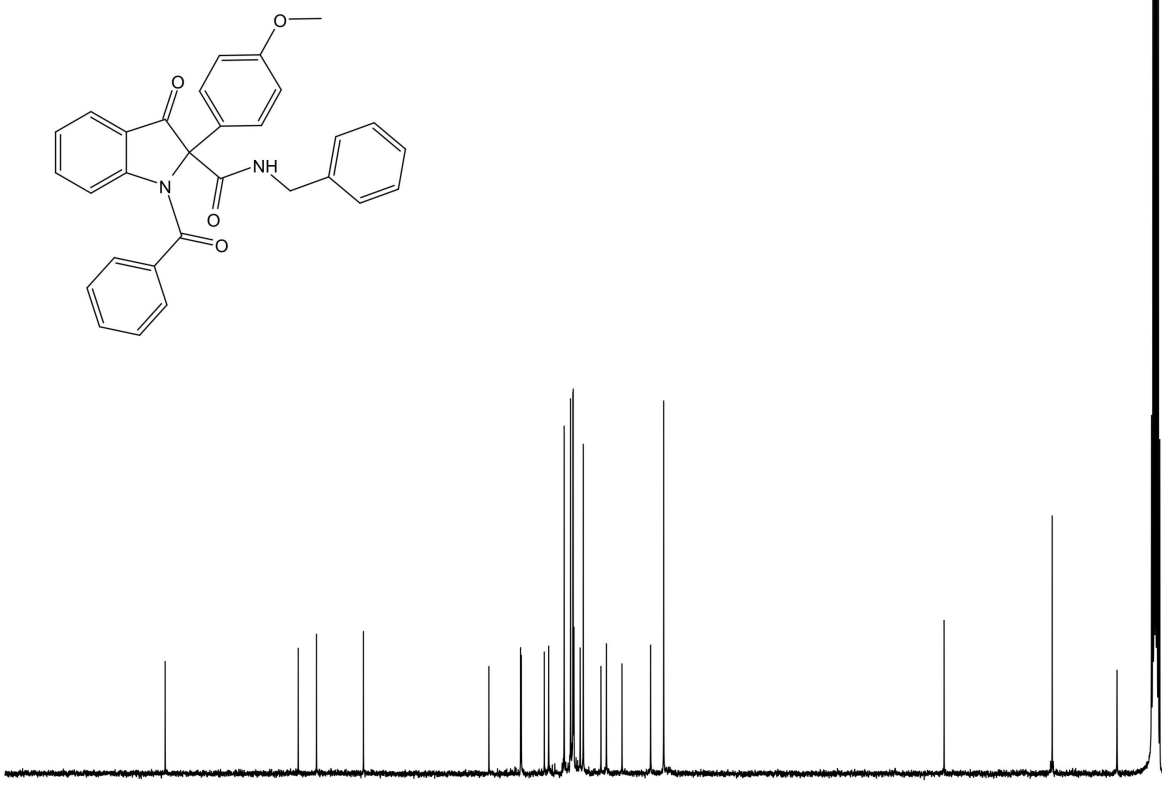

$\begin{array}{llllllllllll}210 & 200 & 190 & 180 & 170 & 160 & 150 & 140 & 130 & 120 & 110 & 100 \\ \mathrm{f} 1(\mathrm{ppm})\end{array}$ 
${ }^{1} \mathrm{H} \mathrm{NMR}$ of Compound $\mathbf{6 c}\left(400 \mathrm{MHz}, \mathrm{CDCl}_{3}\right)$

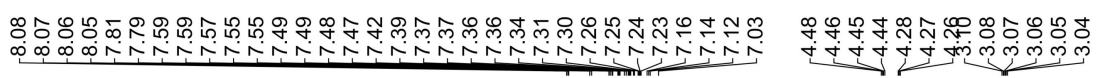
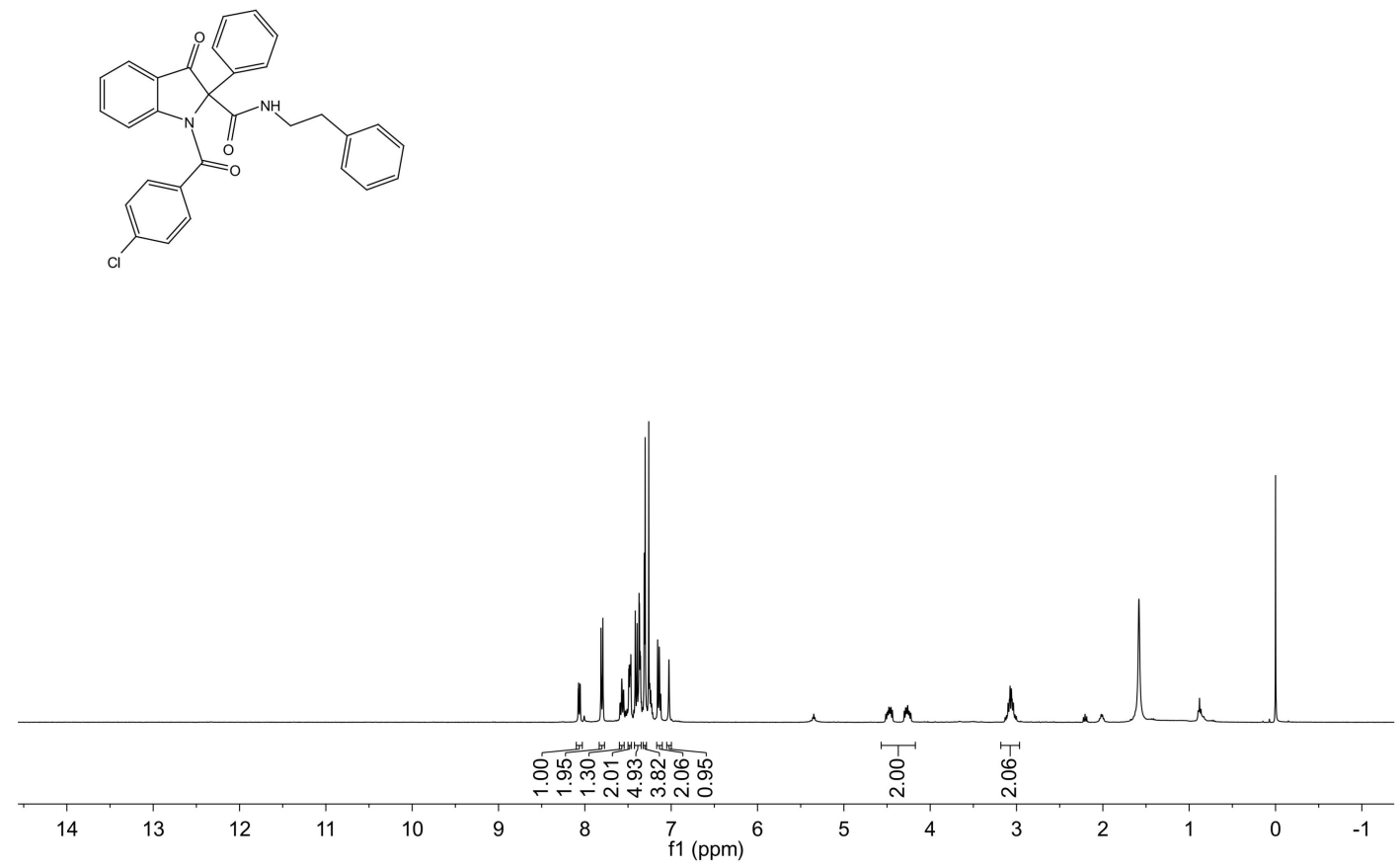

${ }^{13} \mathrm{C}\left\{{ }^{1} \mathrm{H}\right\}$ NMR of Compound 6c $\left(400 \mathrm{MHz}, \mathrm{CDCl}_{3}\right)$

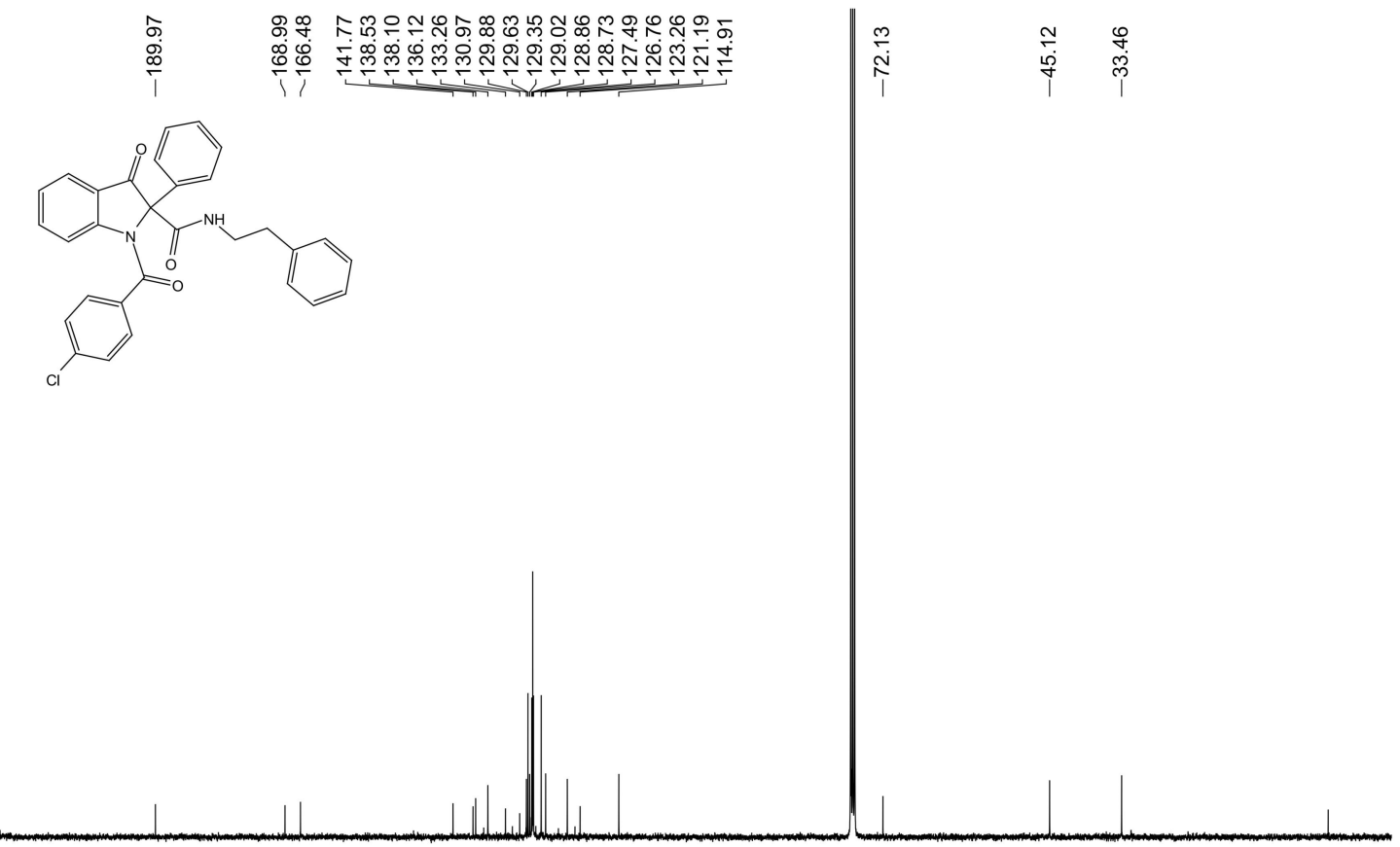

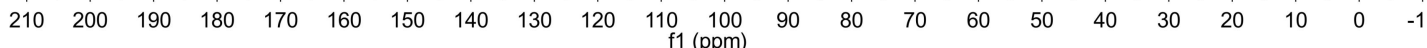


${ }^{1} \mathrm{H}$ NMR of Compound 6d（400 MHz, DMSO-d6 )

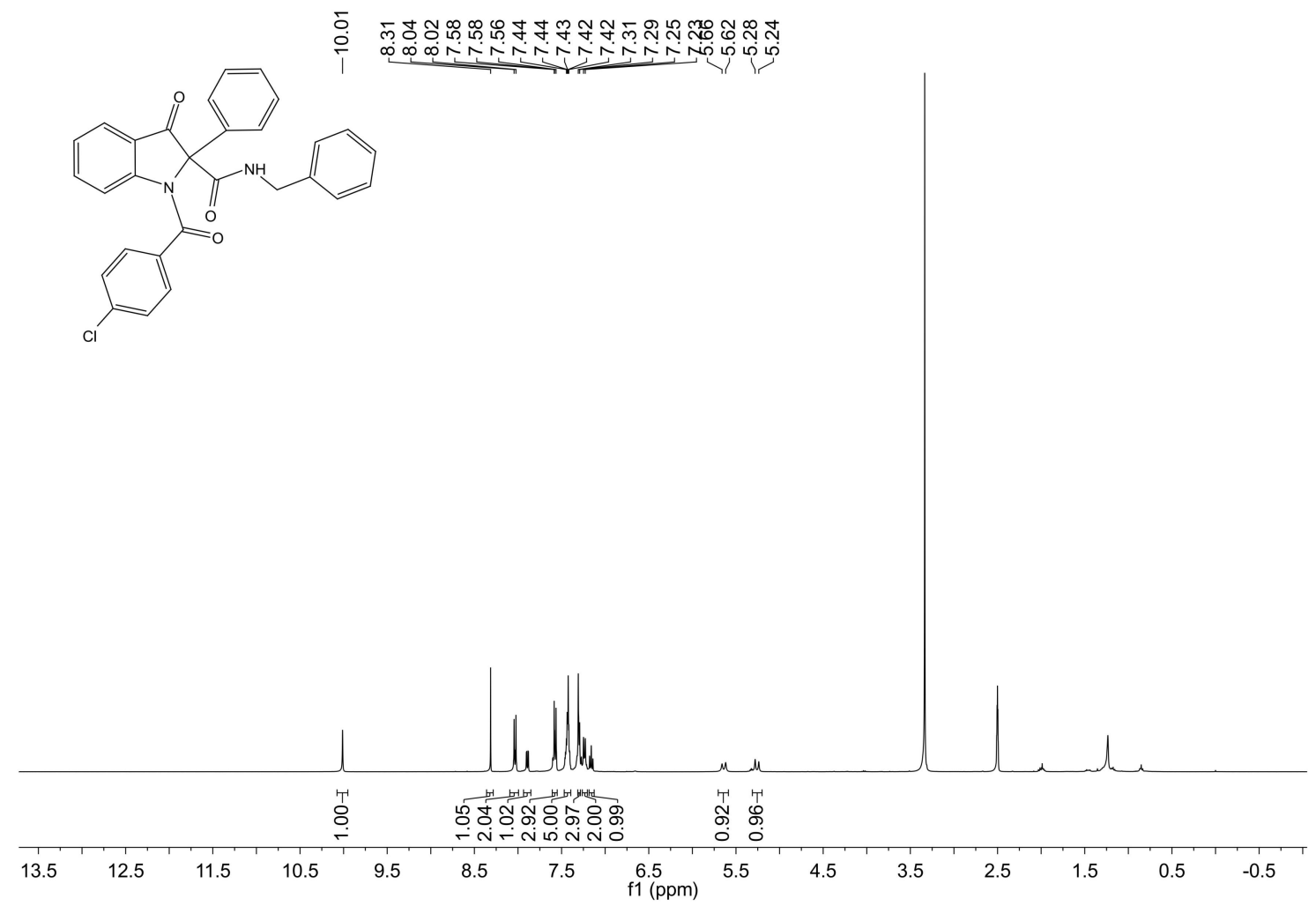

${ }^{13} \mathrm{C}\left\{{ }^{1} \mathrm{H}\right\}$ NMR of Compound 6d (400 MHz, DMSO-d6)
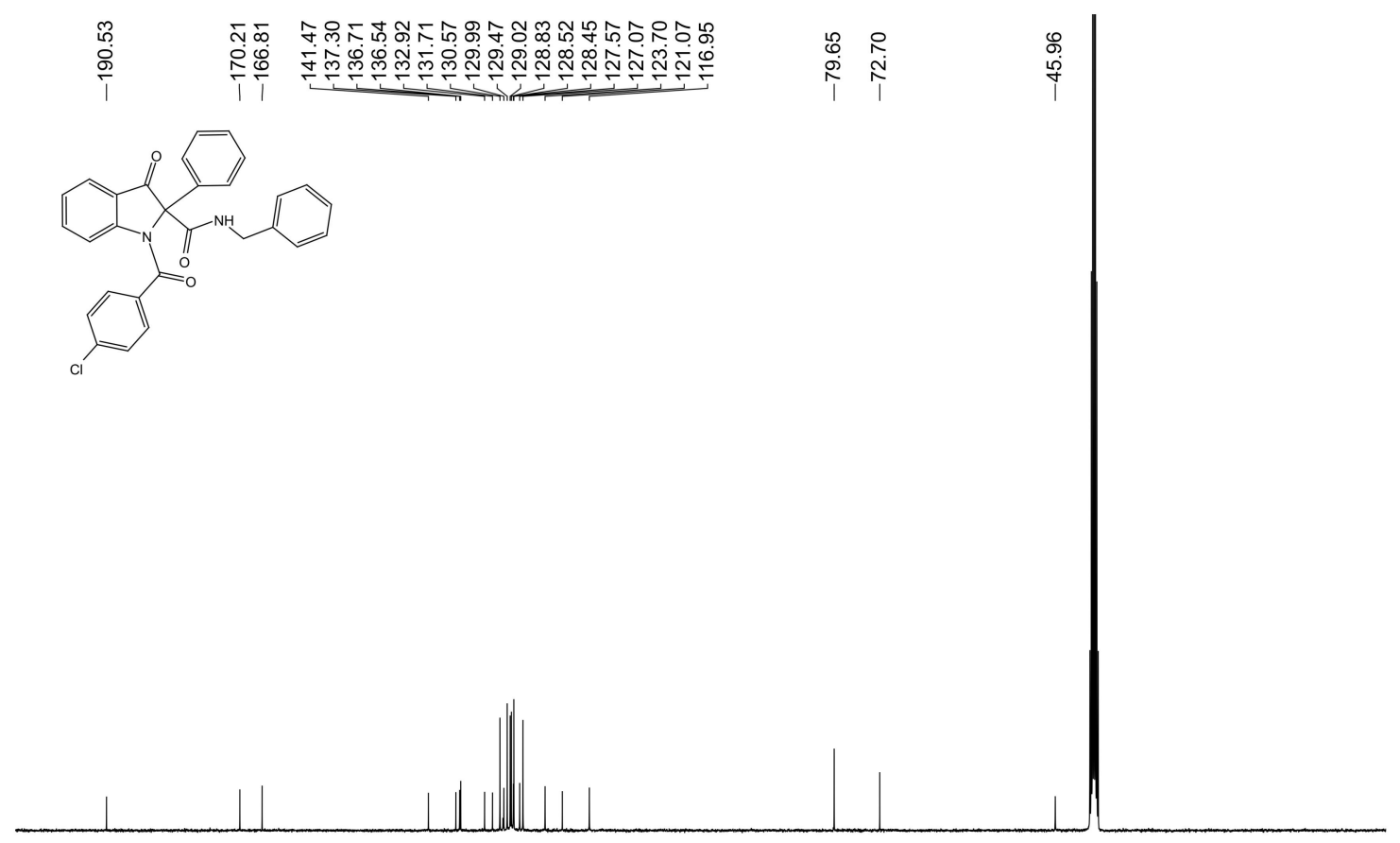

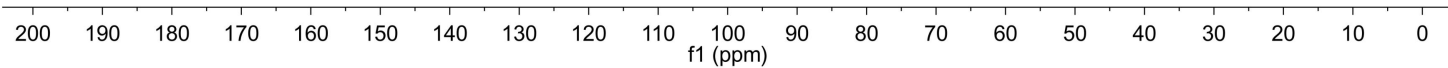


${ }^{1} \mathrm{H} \mathrm{NMR}$ of Compound $\mathbf{6 e}\left(400 \mathrm{MHz}, \mathrm{CDCl}_{3}\right)$

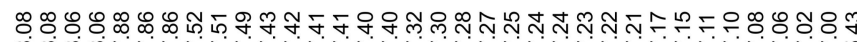

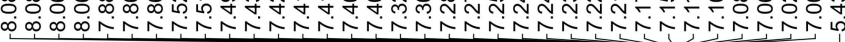

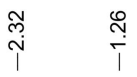

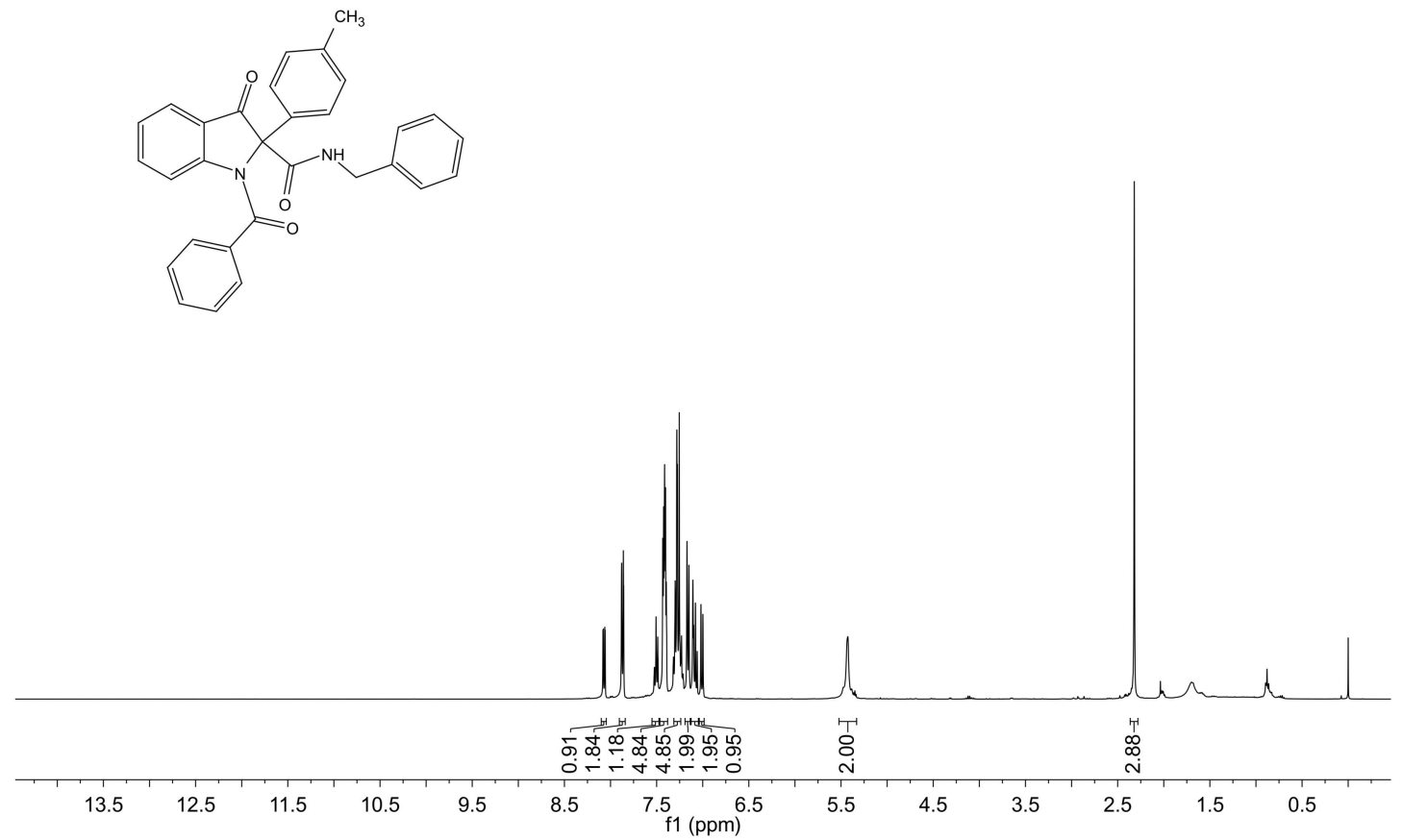

${ }^{13} \mathrm{C}\left\{{ }^{1} \mathrm{H}\right\}$ NMR of Compound $6 \mathbf{e}\left(100 \mathrm{MHz}, \mathrm{CDCl}_{3}\right)$

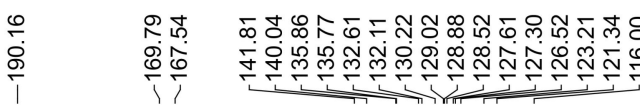

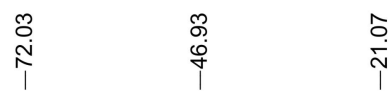
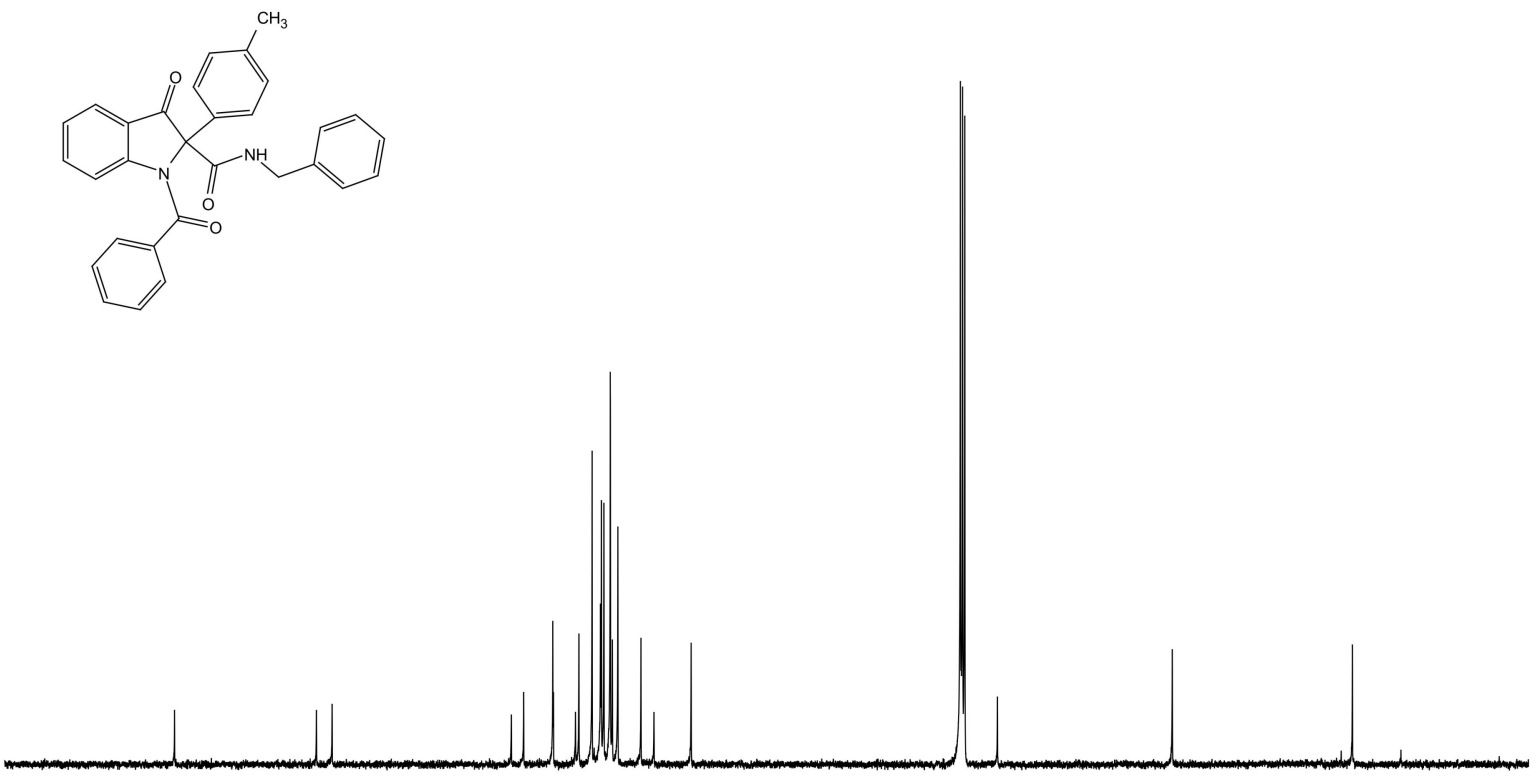

$\begin{array}{lllllllllll}210 & 200 & 190 & 180 & 170 & 160 & 150 & 140 & 130 & 120 & \begin{array}{l}110 \\ \mathrm{f} 1(\mathrm{ppm})\end{array}\end{array}$ 
${ }^{1} \mathrm{H}$ NMR of Compound $6 \mathbf{f}\left(400 \mathrm{MHz}, \mathrm{CDCl}_{3}\right.$ )

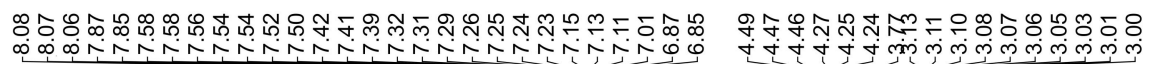
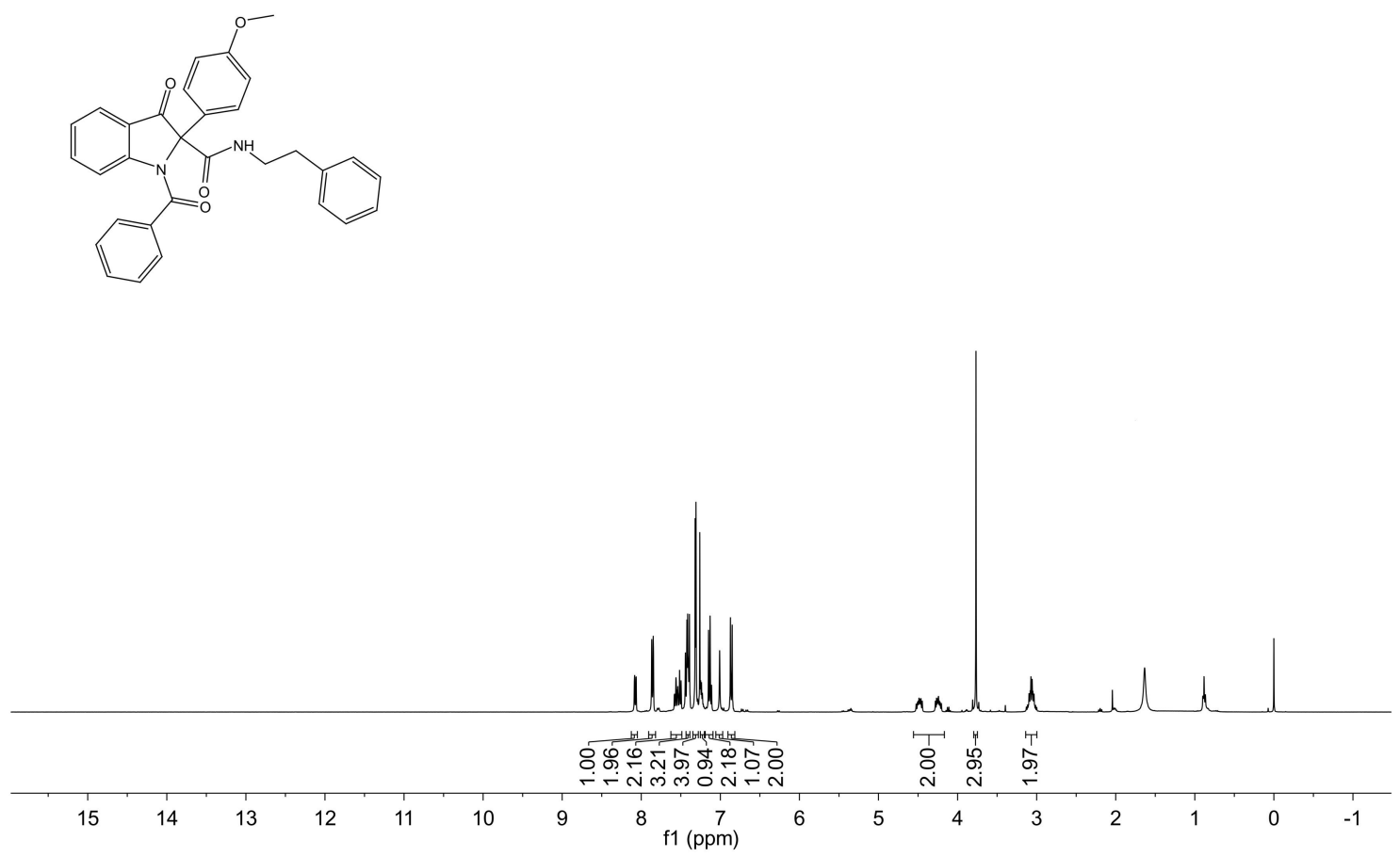

${ }^{13} \mathrm{C}\left\{{ }^{1} \mathrm{H}\right\}$ NMR of Compound $6 \mathbf{f}\left(100 \mathrm{MHz}, \mathrm{CDCl}_{3}\right)$
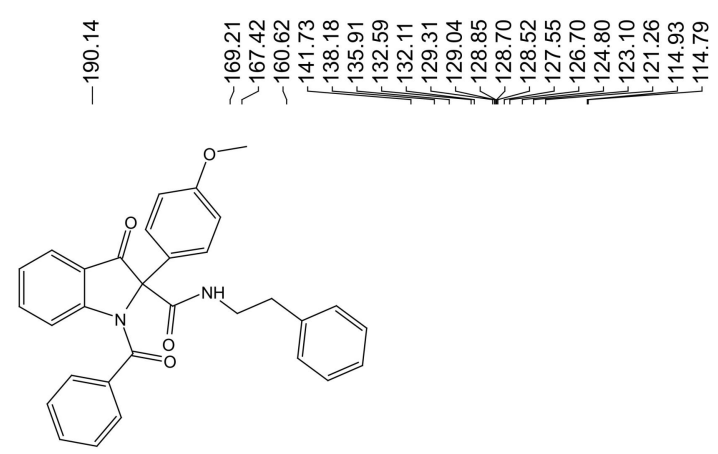

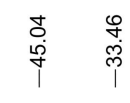
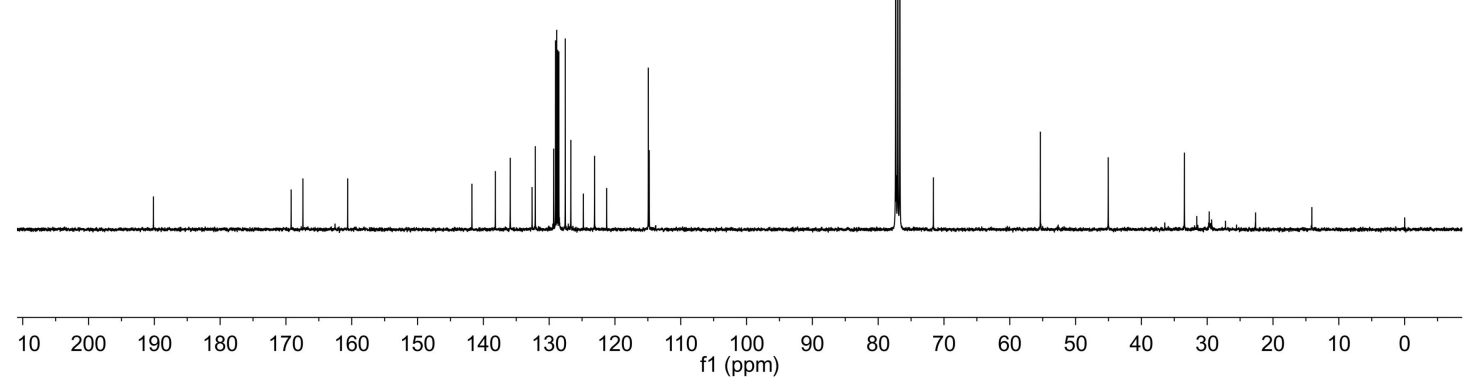

S36 
${ }^{1} \mathrm{H} \mathrm{NMR}$ of Compound $\mathbf{6 g}\left(400 \mathrm{MHz}, \mathrm{CDCl}_{3}\right)$

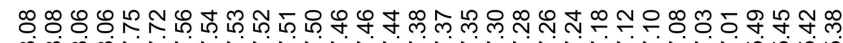

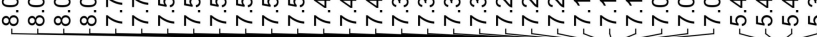
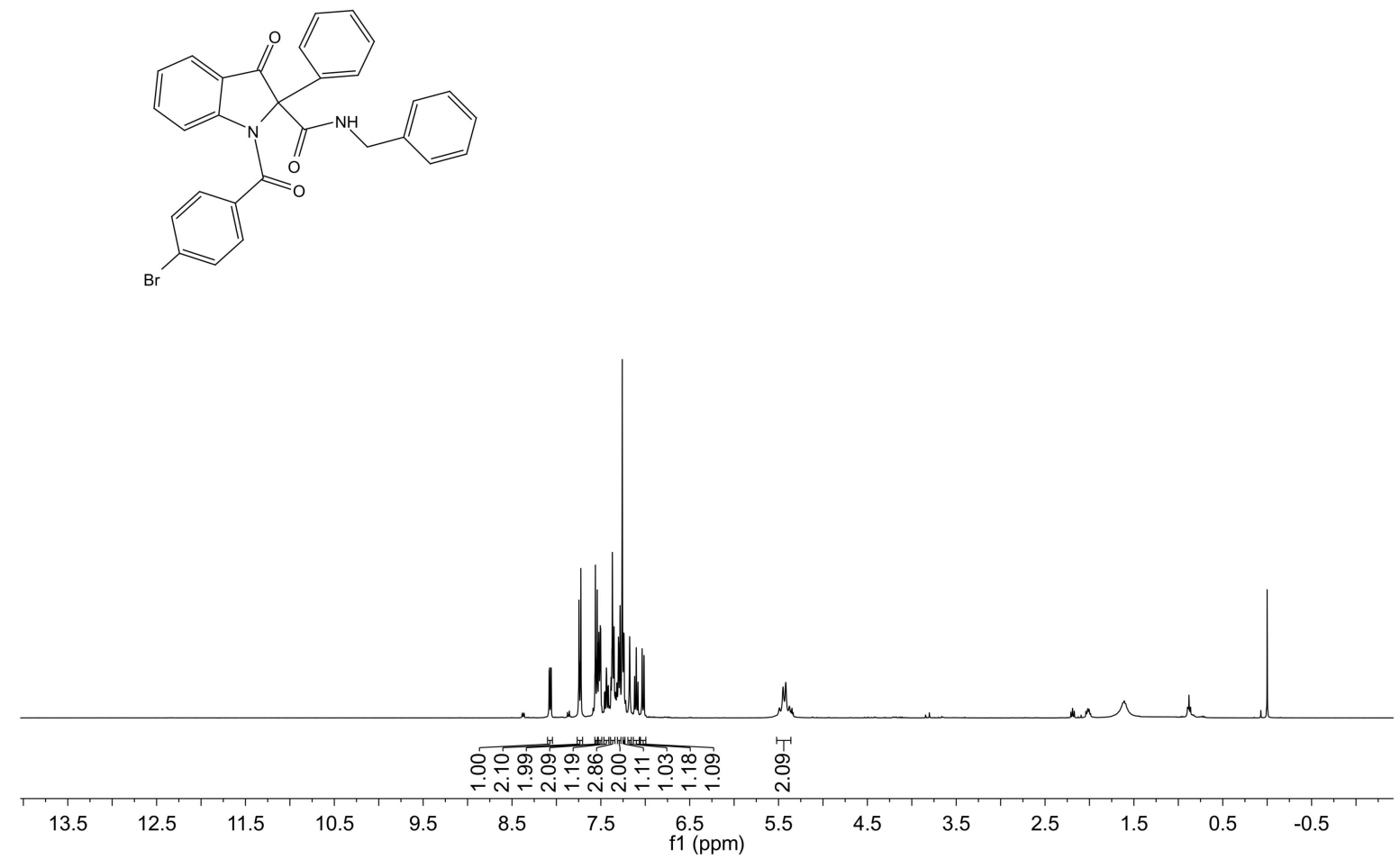

${ }^{13} \mathrm{C}\left\{{ }^{1} \mathrm{H}\right\}$ NMR of Compound $\mathbf{6 g}\left(100 \mathrm{MHz}, \mathrm{CDCl}_{3}\right)$
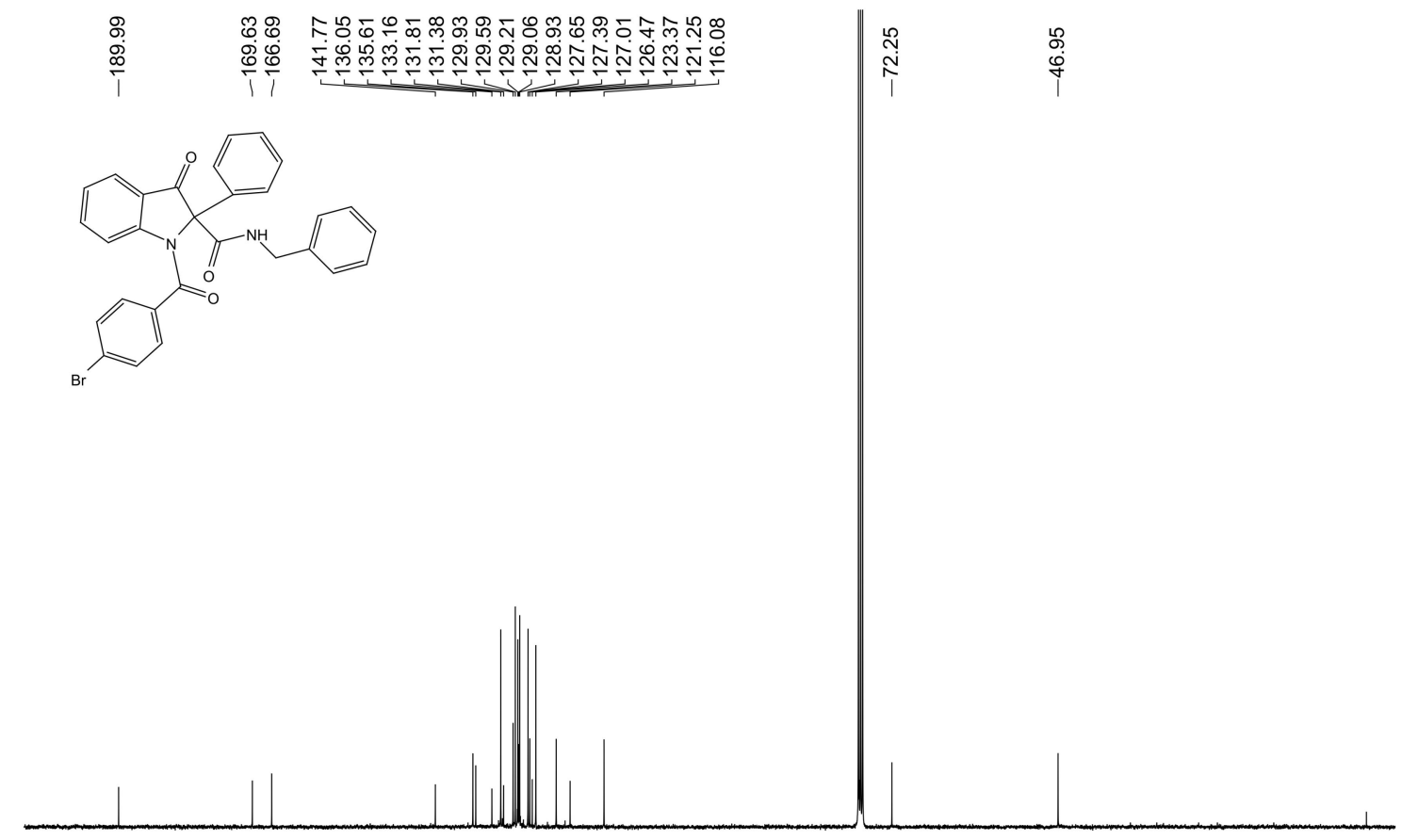

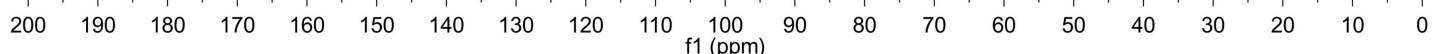


${ }^{1} \mathrm{H} \mathrm{NMR}$ of Compound $\mathbf{6 h}\left(400 \mathrm{MHz}, \mathrm{CDCl}_{3}\right)$
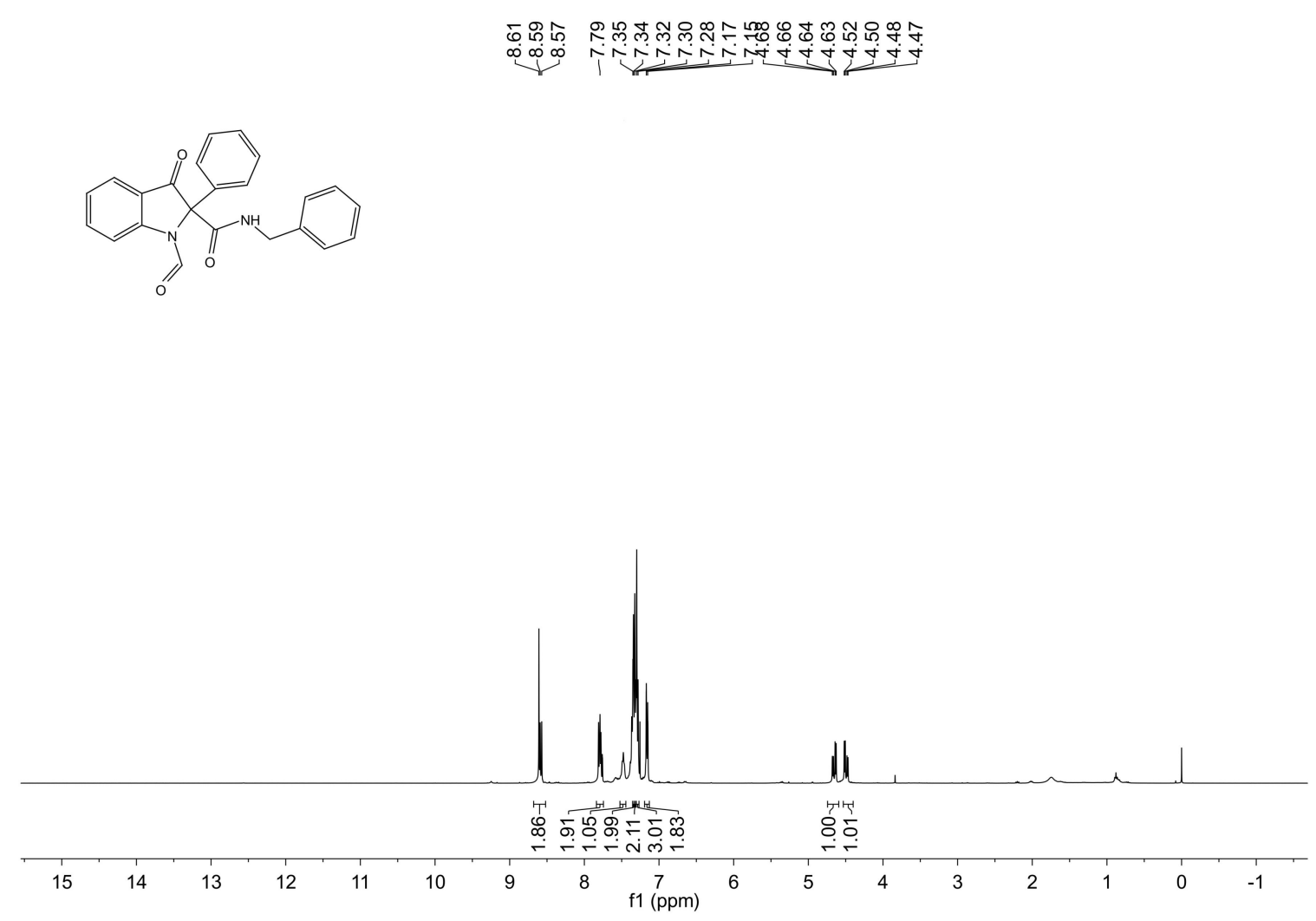

${ }^{13} \mathrm{C}\left\{{ }^{1} \mathrm{H}\right\}$ NMR of Compound $\mathbf{6 h}\left(100 \mathrm{MHz}, \mathrm{CDCl}_{3}\right)$
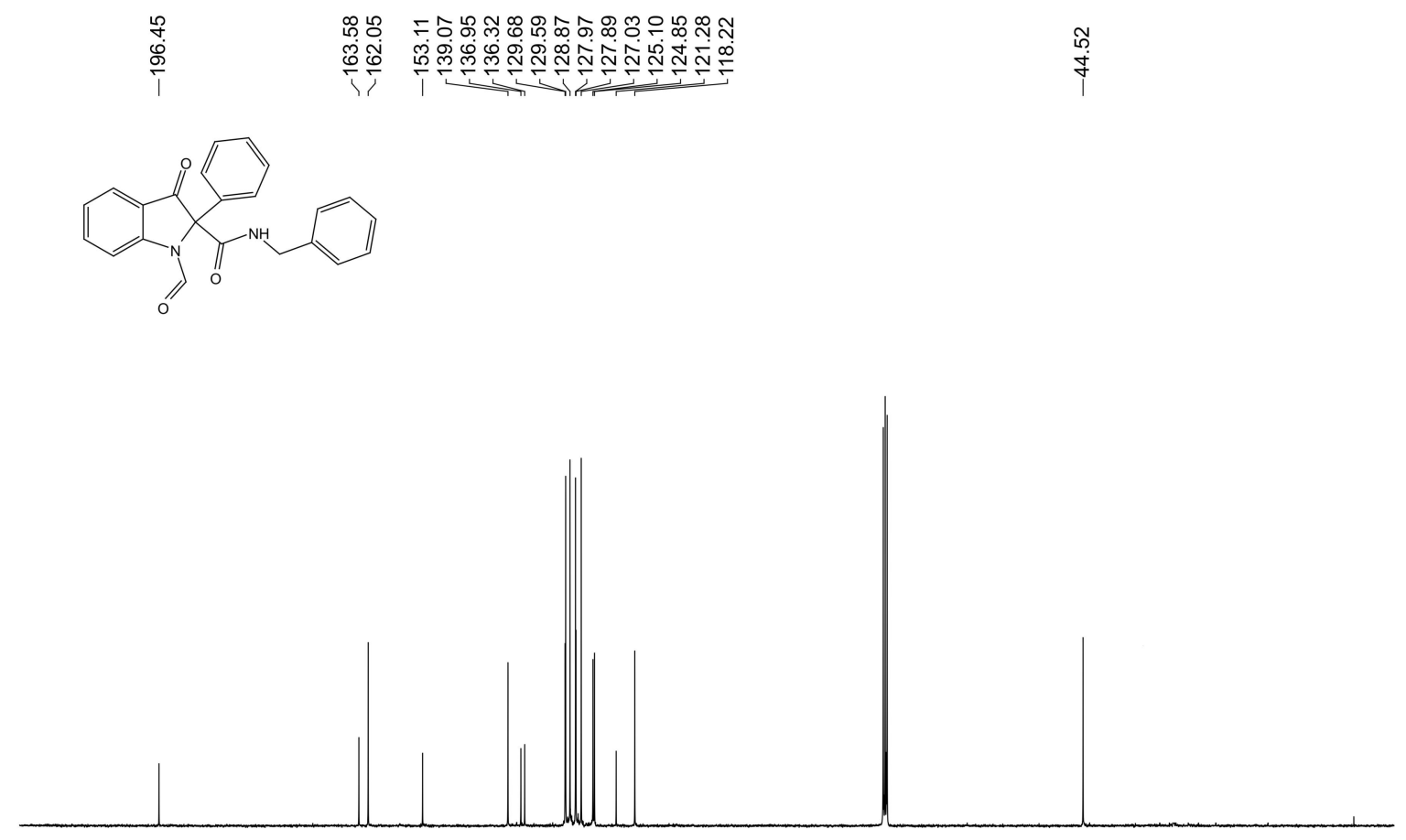

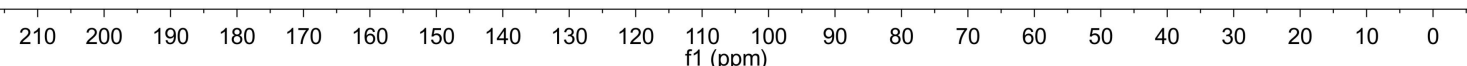


${ }^{1} \mathrm{H}$ NMR of Compound $6 \mathbf{i}\left(400 \mathrm{MHz}, \mathrm{CDCl}_{3}\right)$

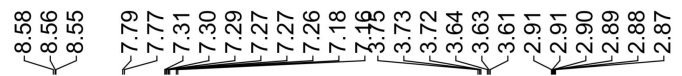
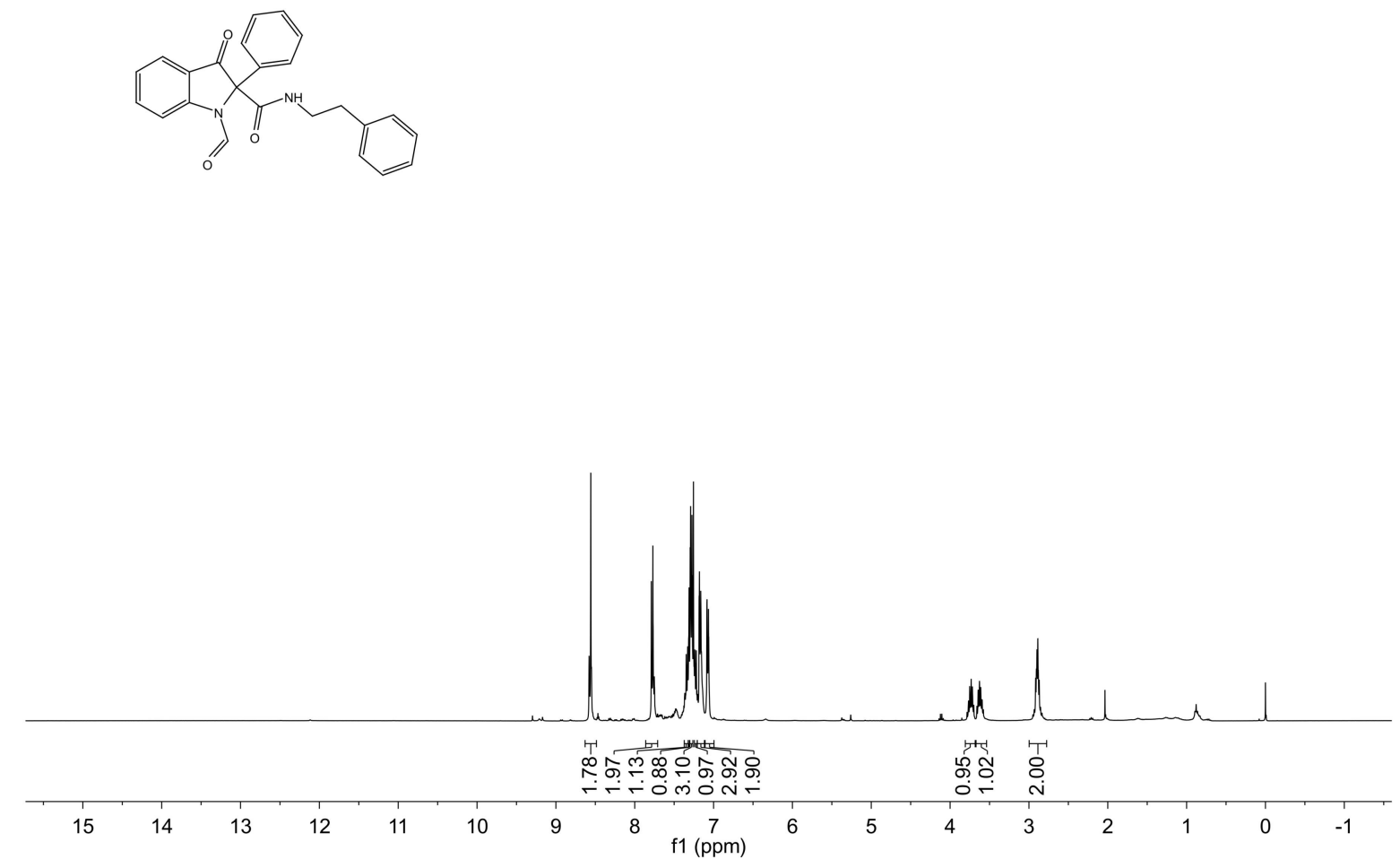

${ }^{13} \mathrm{C}\left\{{ }^{1} \mathrm{H}\right\}$ NMR of Compound $6 \mathbf{i}\left(100 \mathrm{MHz}, \mathrm{CDCl}_{3}\right)$

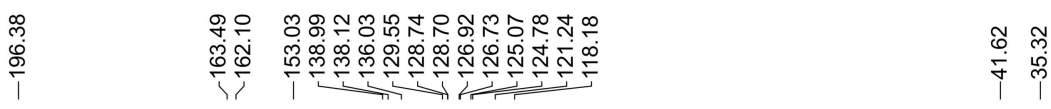
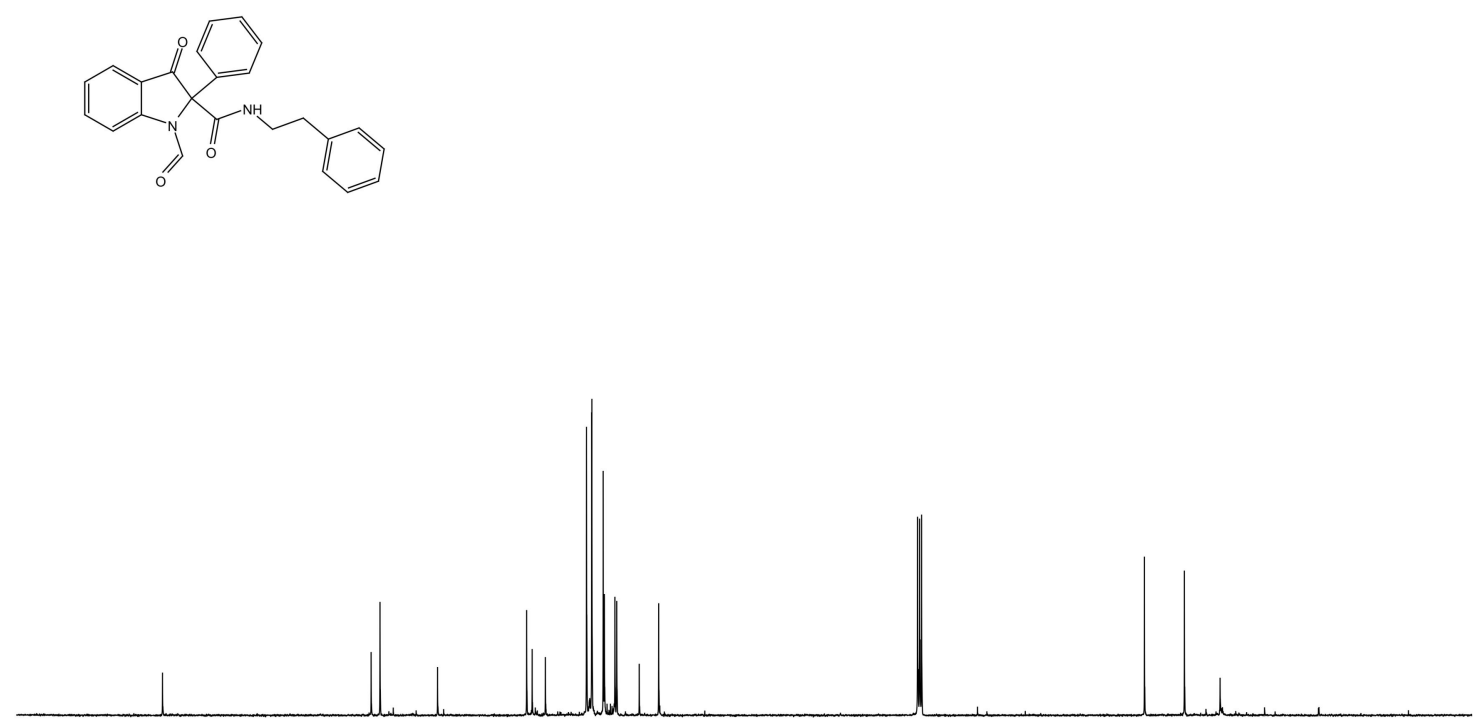

$\begin{array}{lllllllllllllllllllllllll}210 & 200 & 190 & 180 & 170 & 160 & 150 & 140 & 130 & 120 & 110 & 100 & 90 & 80 & 70 & 60 & 50 & 40 & 30 & 20 & 10 & 0 & -1\end{array}$ 
${ }^{1} \mathrm{H} \mathrm{NMR}$ of Compound $\mathbf{6 j}\left(400 \mathrm{MHz}, \mathrm{CDCl}_{3}\right)$

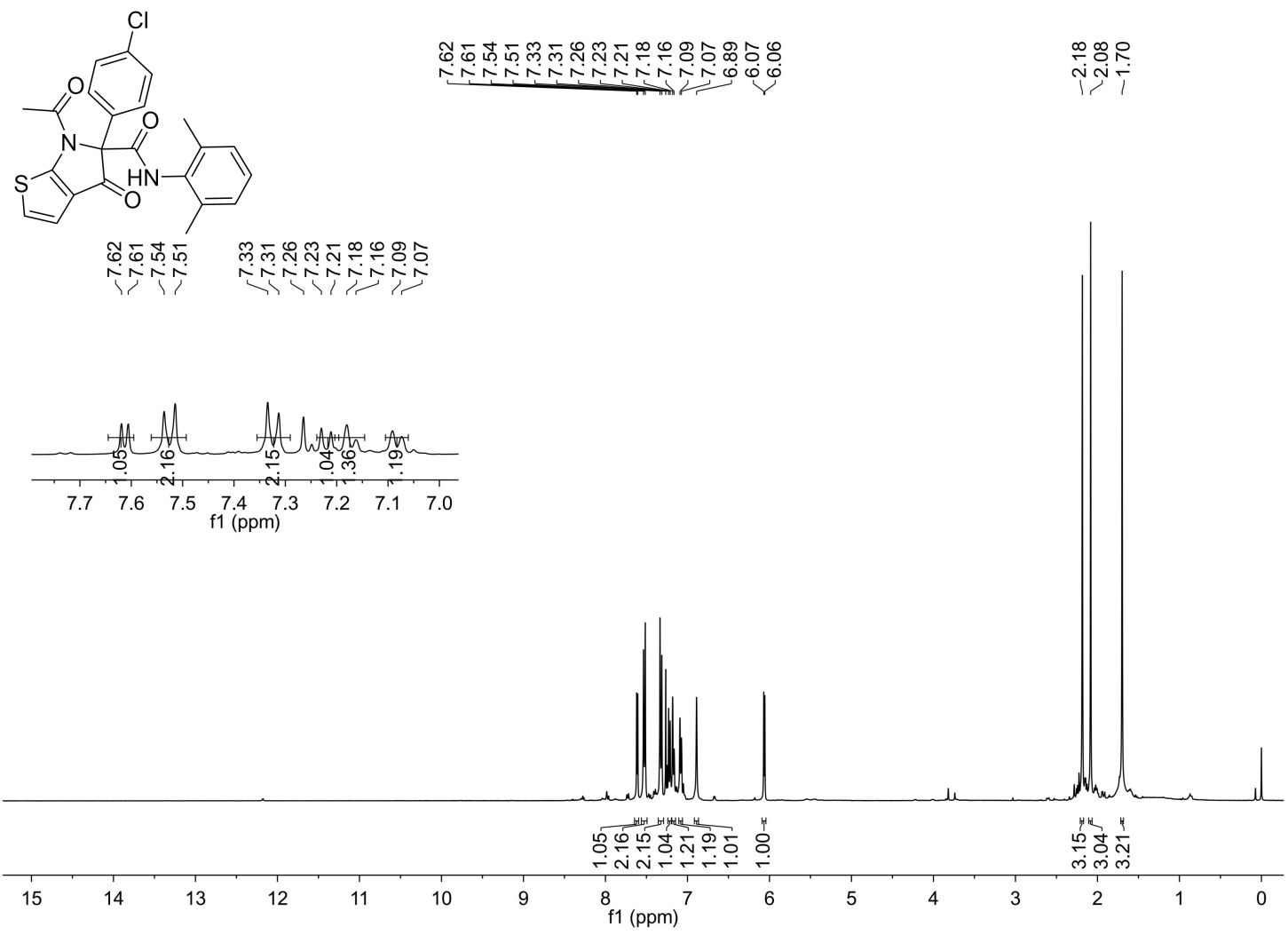

${ }^{13} \mathrm{C}\left\{{ }^{1} \mathrm{H}\right\}$ NMR of Compound $\mathbf{6 j}\left(100 \mathrm{MHz}, \mathrm{CDCl}_{3}\right)$
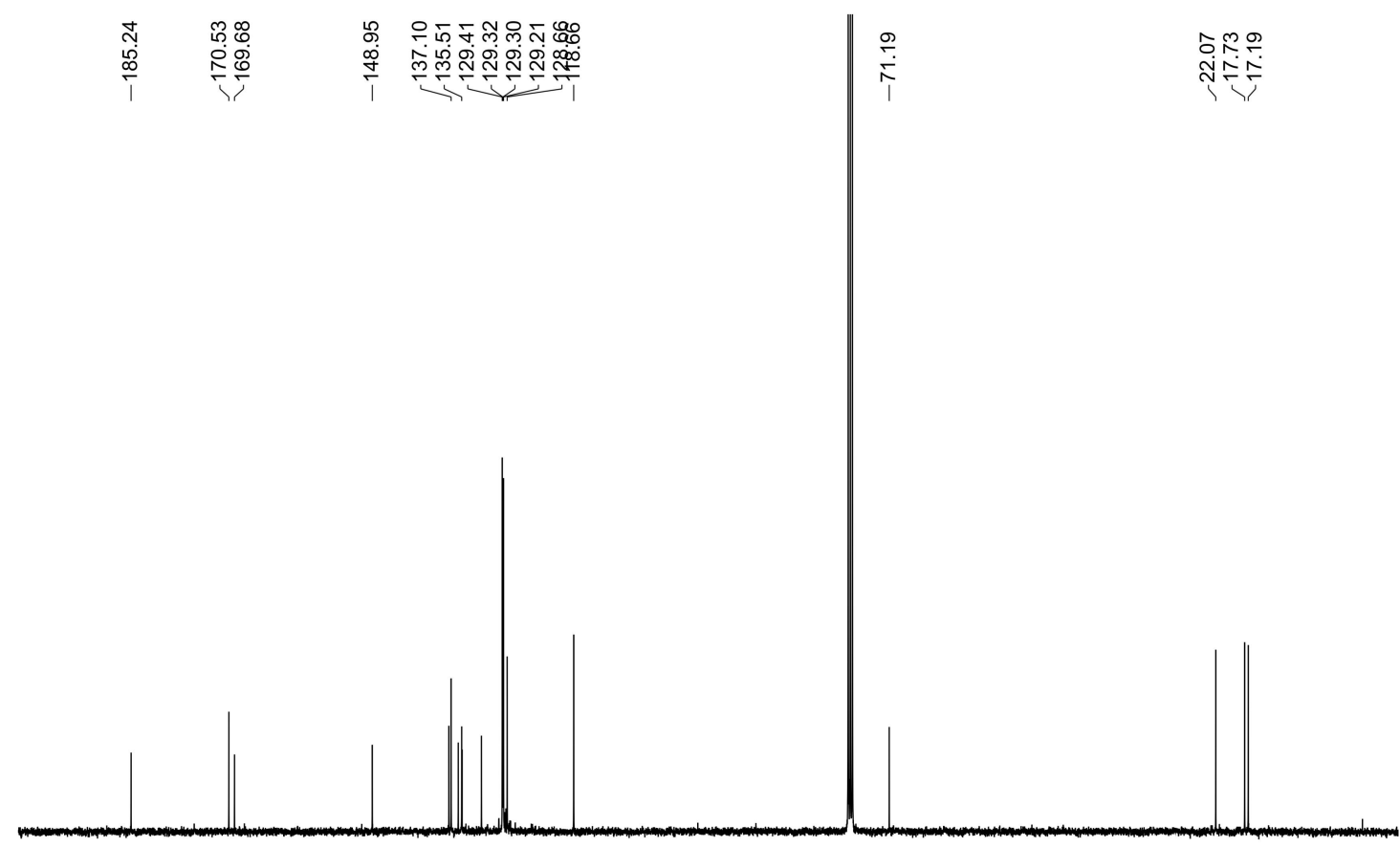

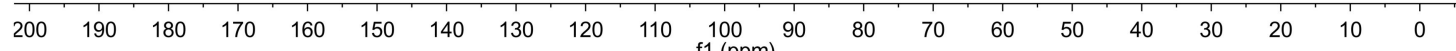


${ }^{1} \mathrm{H}$ NMR ofCompound $\mathbf{6 k} \quad\left(400 \mathrm{MHz}, \mathrm{CDCl}_{3}\right)$

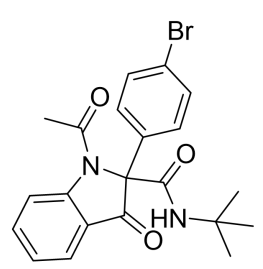

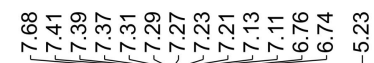

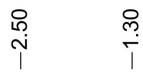

?

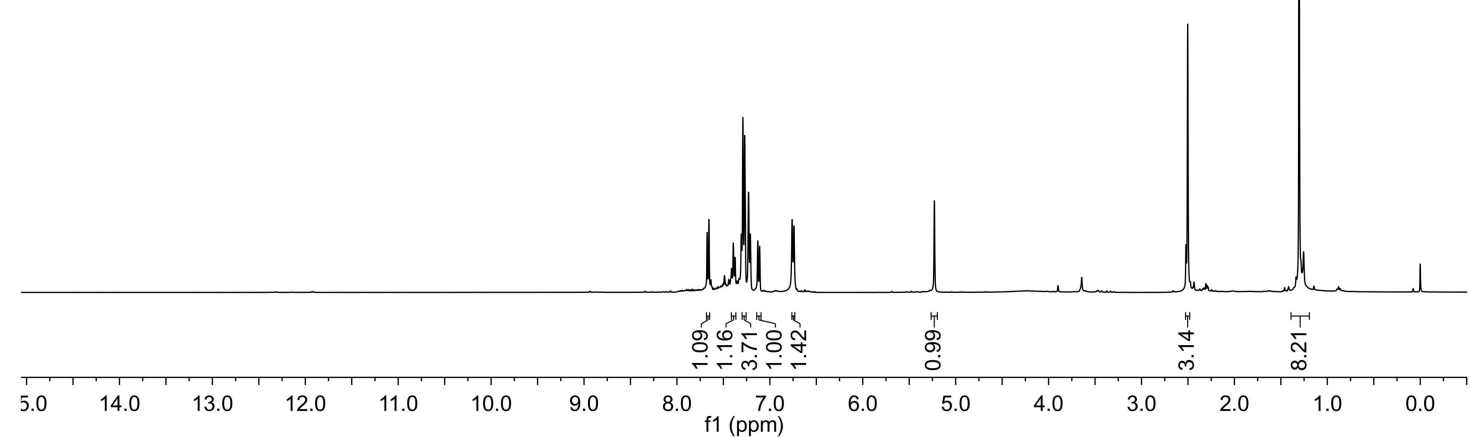

${ }^{13} \mathrm{C}\left\{{ }^{1} \mathrm{H}\right\}$ NMR of Compound 6k $\left(100 \mathrm{MHz}, \mathrm{CDCl}_{3}\right)$

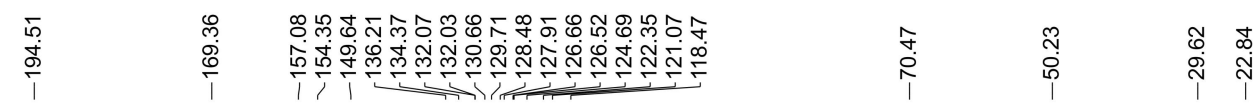

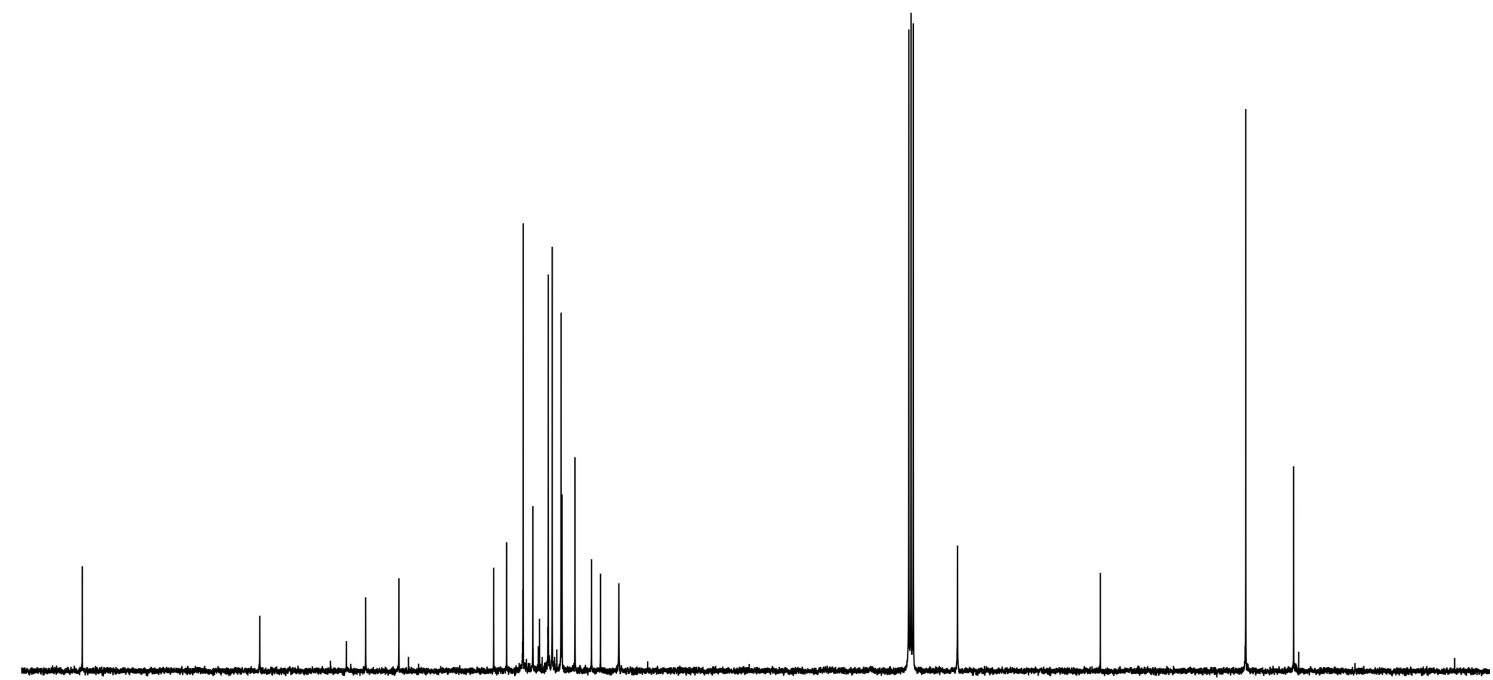

$\begin{array}{lllllllllllllllllllll}200 & 190 & 180 & 170 & 160 & 150 & 140 & 130 & 120 & 110 & \begin{array}{c}100 \\ 1\end{array} & 90 & 80 & 70 & 60 & 50 & 40 & 30 & 20 & 10 & 0\end{array}$ 
${ }^{1} \mathrm{H}$ NMR of Compound 10a $\left(400 \mathrm{MHz}, \mathrm{CDCl}_{3}\right)$

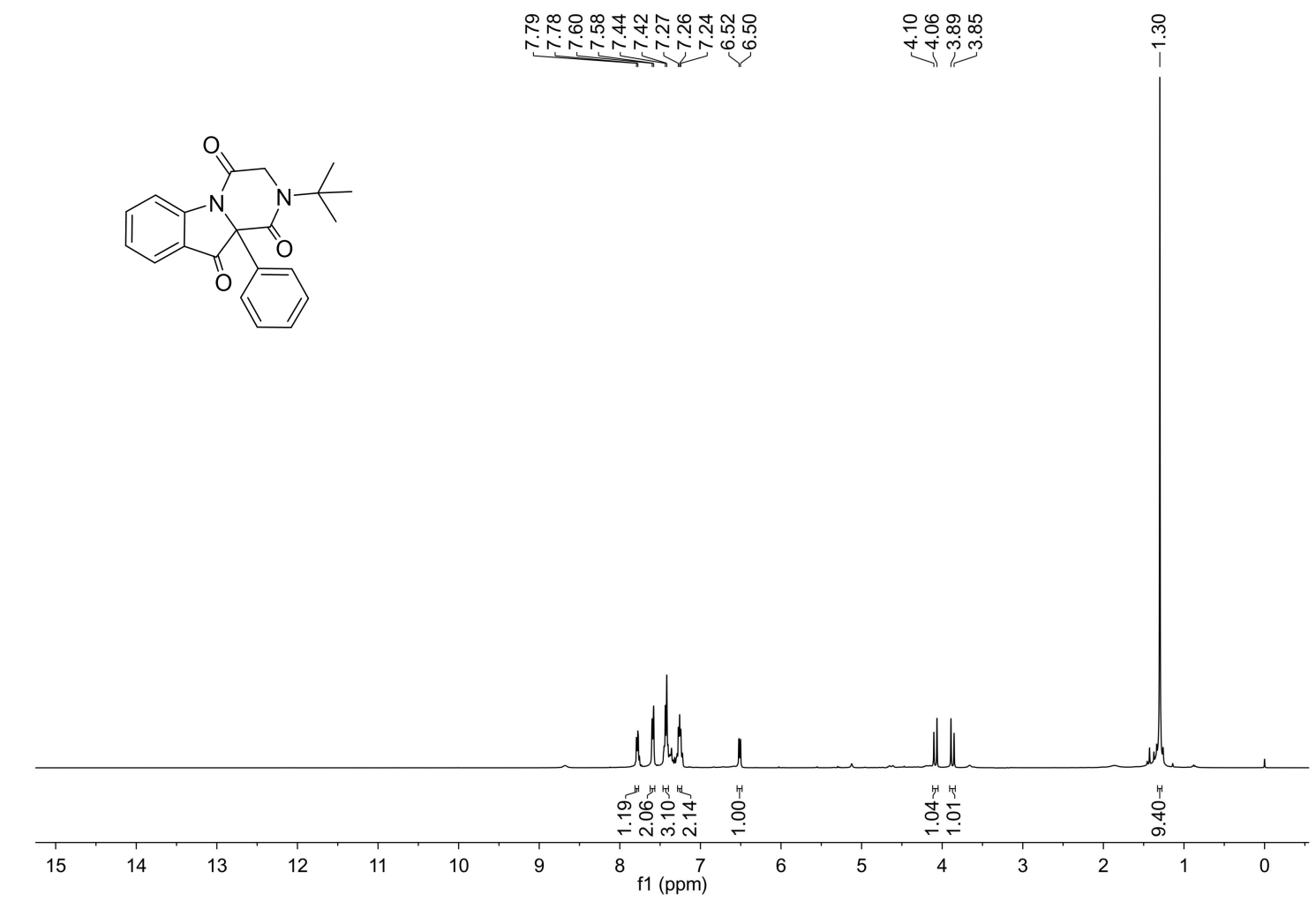

${ }^{13} \mathrm{C}\left\{{ }^{1} \mathrm{H}\right\}$ NMR of Compound 10a $\left(100 \mathrm{MHz}, \mathrm{CDCl}_{3}\right)$

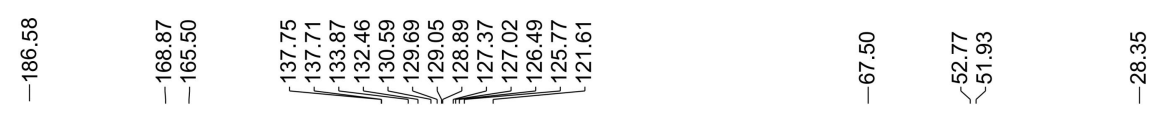

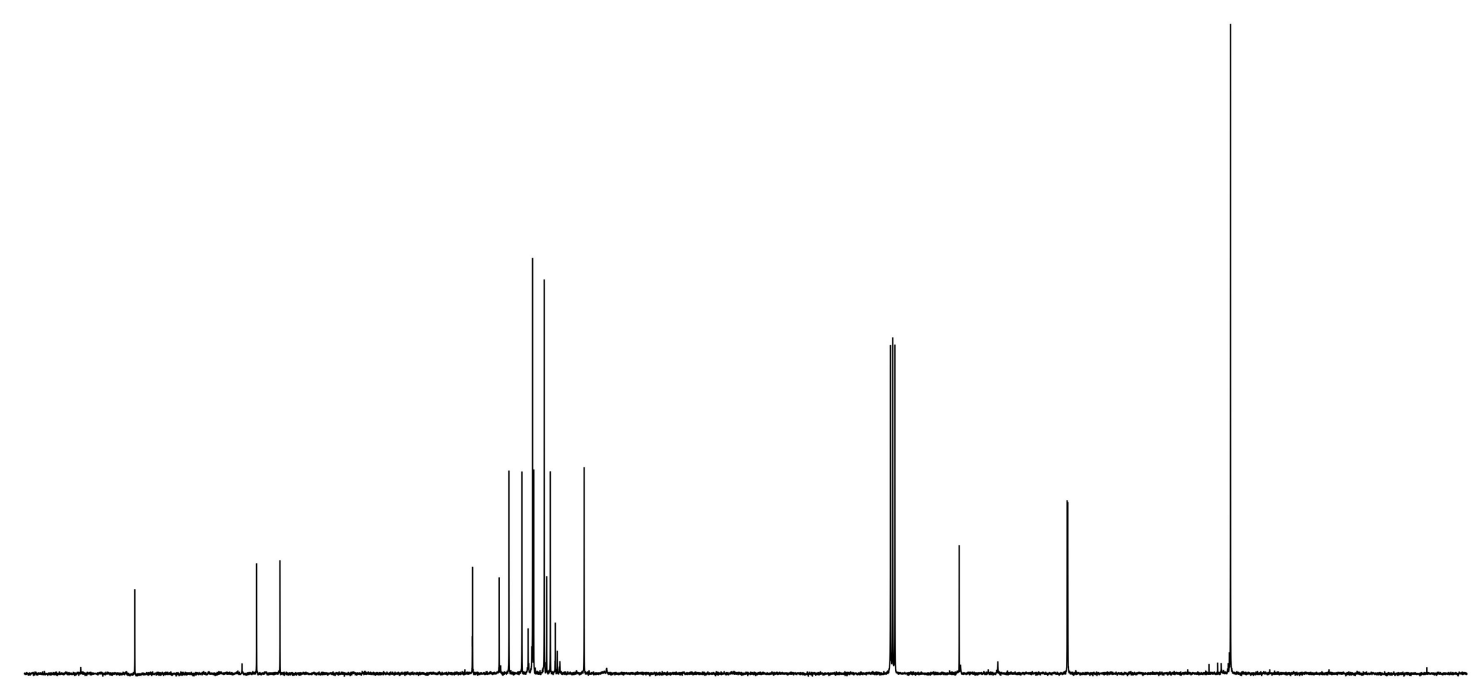

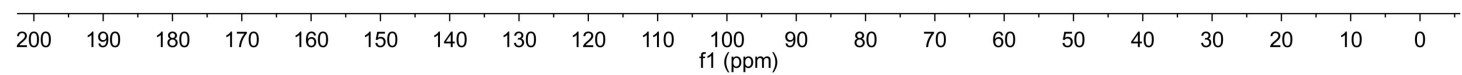


${ }^{1} \mathrm{H}$ NMR of Compound 10b (400 MHz, $\left.\mathrm{CDCl}_{3}\right)$

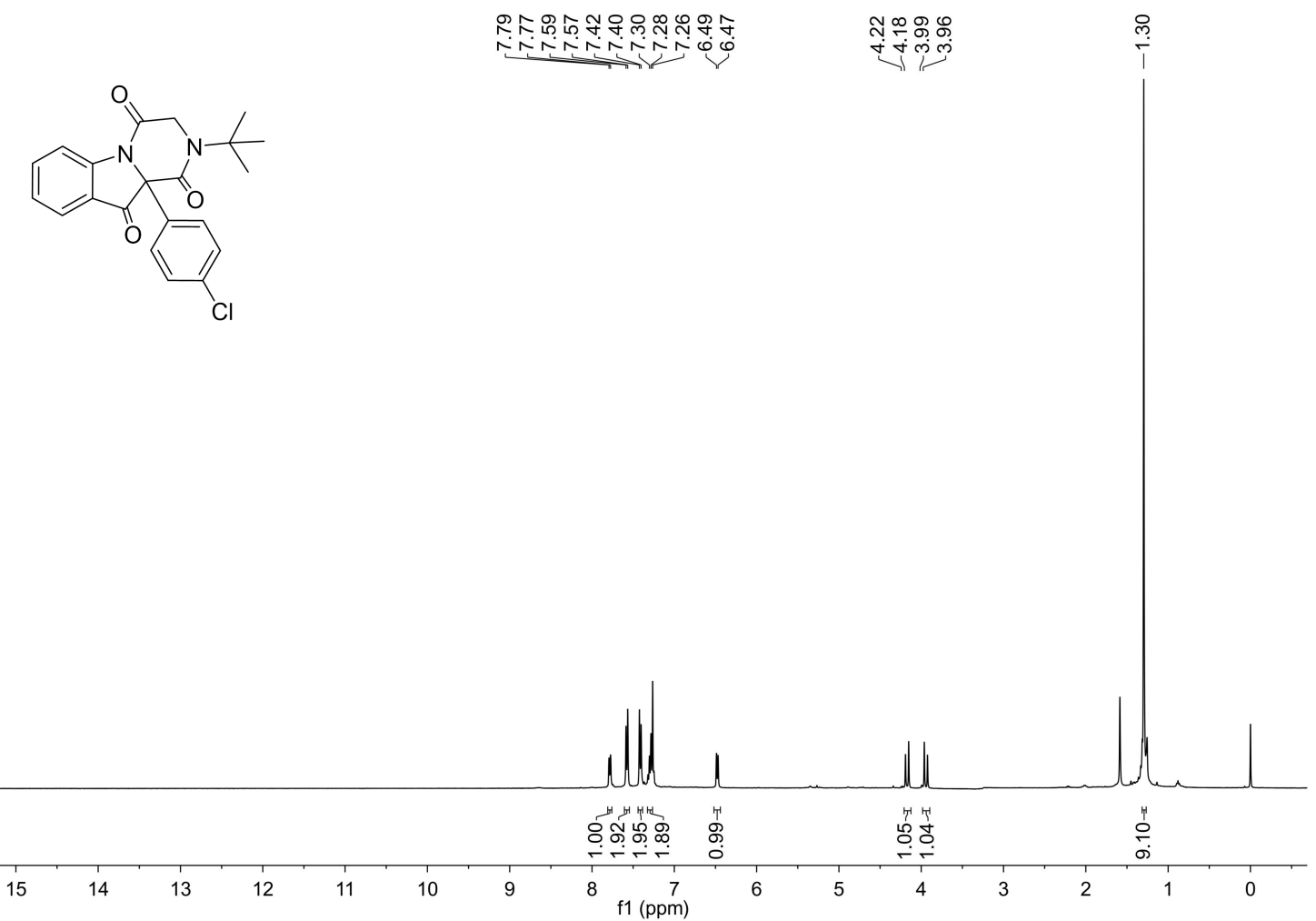

${ }^{13} \mathrm{C}\left\{{ }^{1} \mathrm{H}\right\}$ NMR of Compound 10b $\left(100 \mathrm{MHz}, \mathrm{CDCl}_{3}\right)$

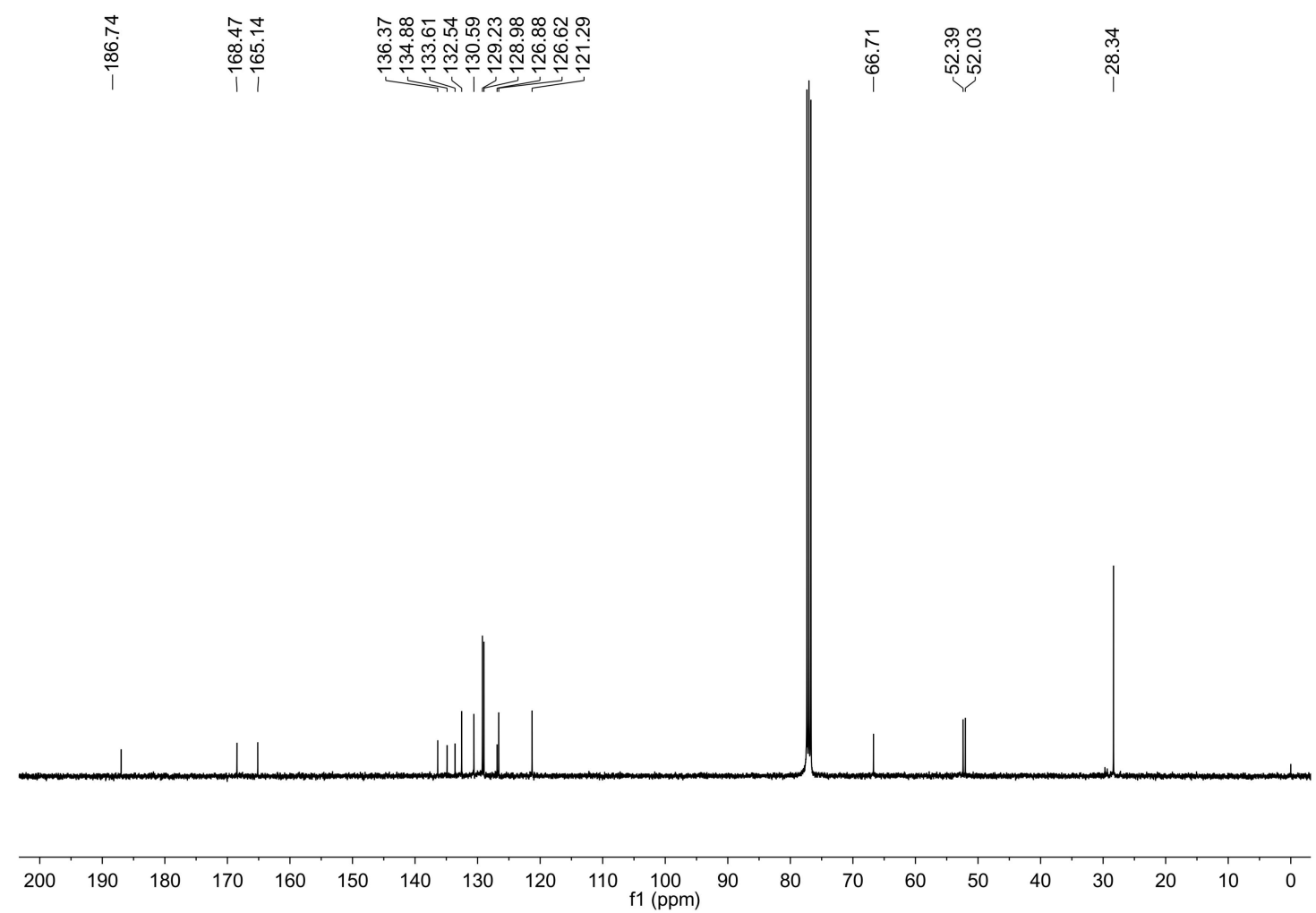


${ }^{1} \mathrm{H}$ NMR of Compound 10c $\left(400 \mathrm{MHz}, \mathrm{CDCl}_{3}\right)$

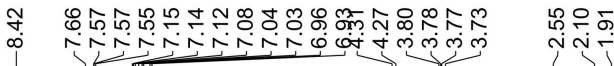<smiles>COc1ccc(C23C(=O)N(c4c(C)cccc4C)CC(=O)N2C3=O)cc1</smiles>

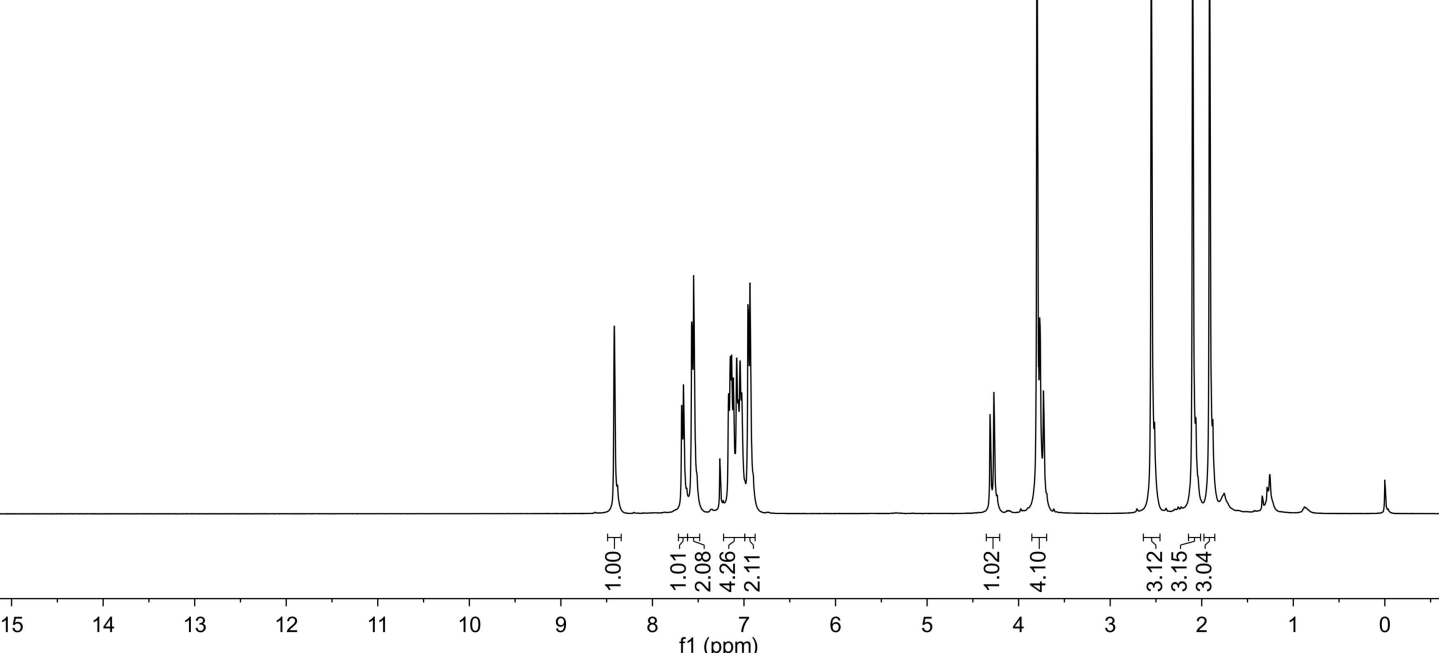

${ }^{13} \mathrm{C}\left\{{ }^{1} \mathrm{H}\right\}$ NMR of Compound 10c $\left(100 \mathrm{MHz}, \mathrm{CDCl}_{3}\right)$

\begin{tabular}{|c|c|c|c|}
\hline 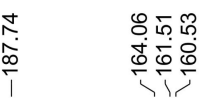 & 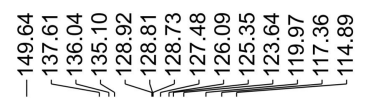 & $\stackrel{\leftrightarrow}{i}$ & 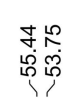 \\
\hline
\end{tabular}

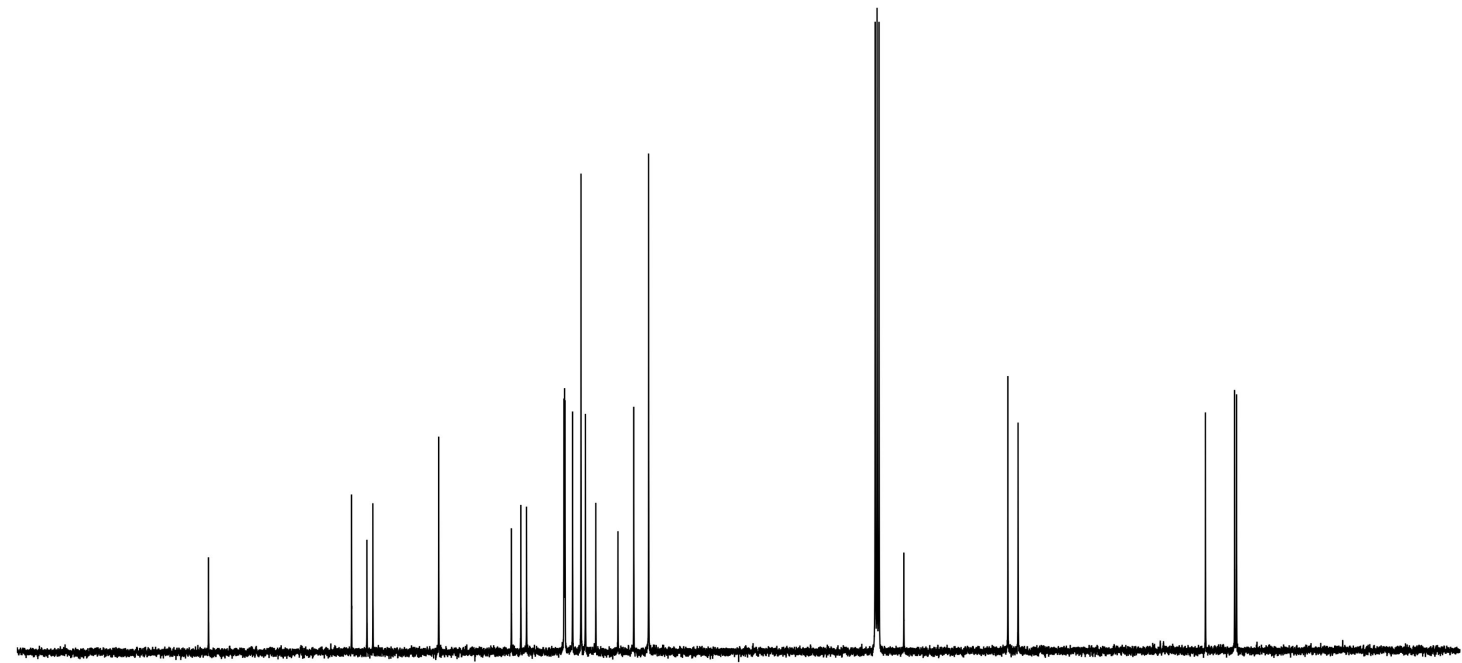

$\begin{array}{lllllllllllllllllllllll}210 & 200 & 190 & 180 & 170 & 160 & 150 & 140 & 130 & 120 & 110 & 100 & 90 & 80 & 70 & 60 & 50 & 40 & 30 & 20 & 10 & 0 & -10\end{array}$ 
${ }^{1} \mathrm{H}$ NMR of Compound 10d $\left(400 \mathrm{MHz}, \mathrm{CDCl}_{3}\right)$

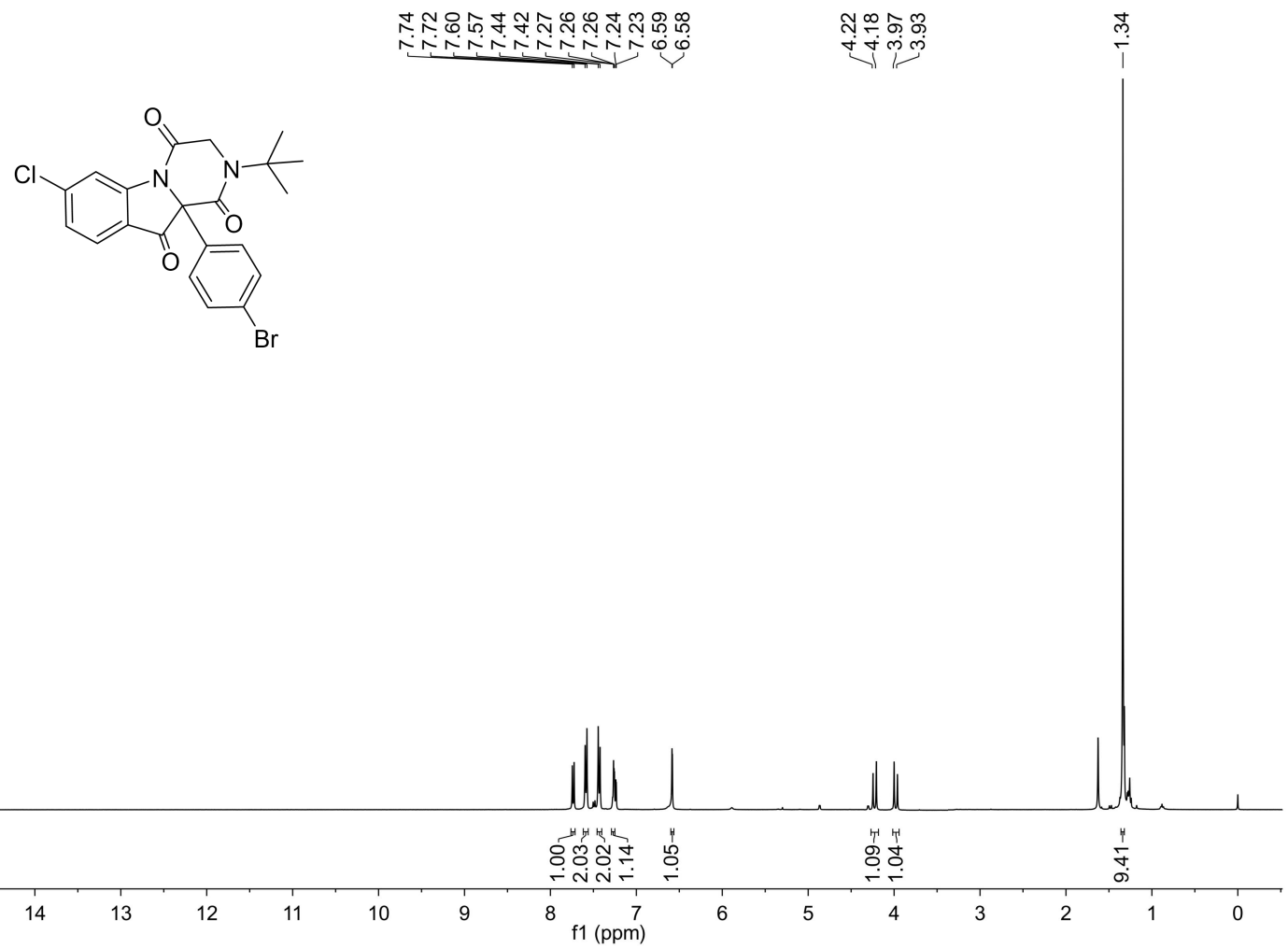

${ }^{13} \mathrm{C}\left\{{ }^{1} \mathrm{H}\right\}$ NMR of Compound 10d $\left(100 \mathrm{MHz}, \mathrm{CDCl}_{3}\right)$

\begin{tabular}{|c|c|c|c|}
\hline \begin{tabular}{l} 
ल) \\
$\infty$ \\
0 \\
0 \\
\hdashline
\end{tabular} & 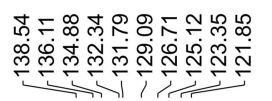 & $\underset{\substack{0 \\
i}}{\stackrel{0}{1}}$ & 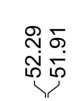 \\
\hline
\end{tabular}

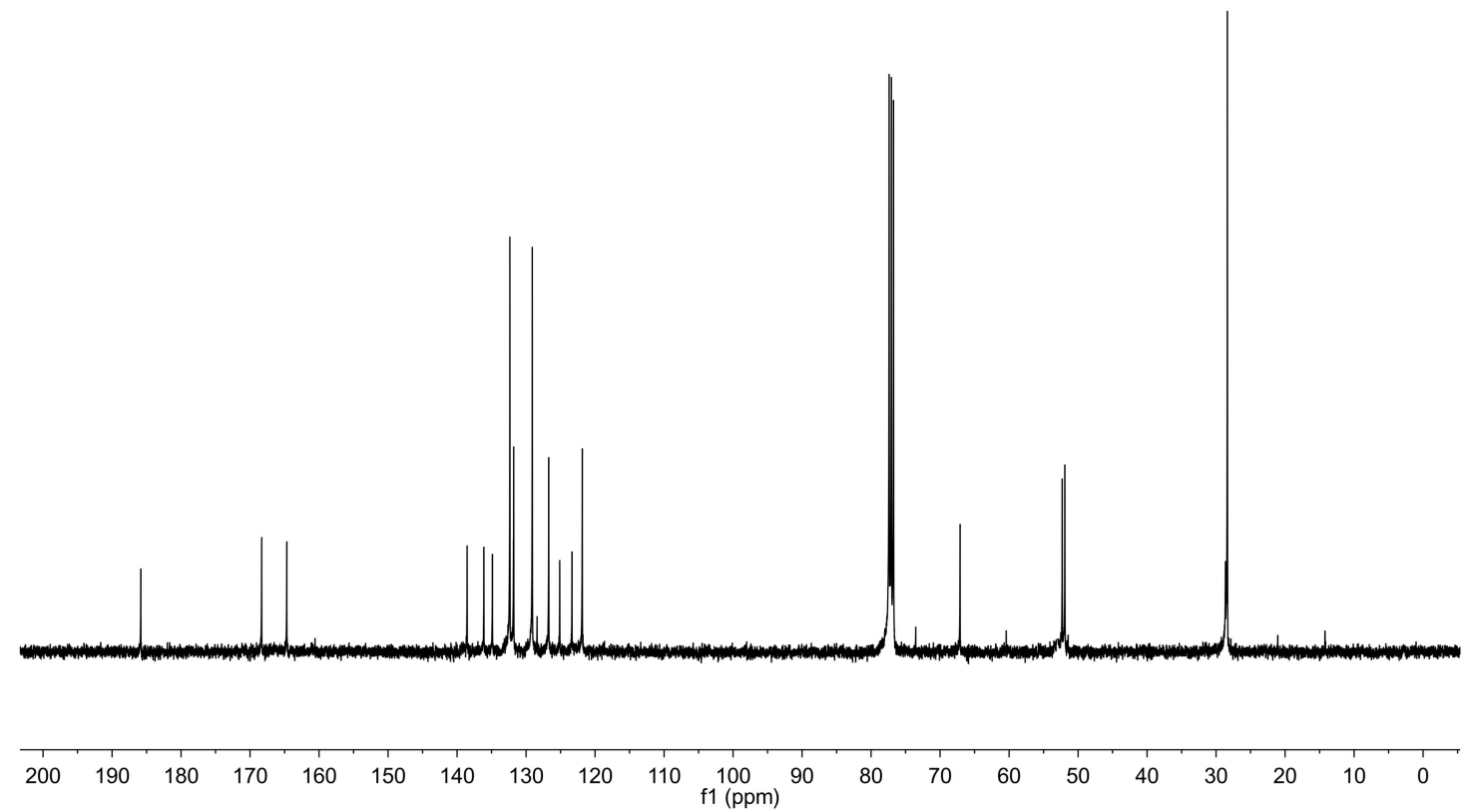


${ }^{1} \mathrm{H}$ NMR of Compound 10e $\left(400 \mathrm{MHz}, \mathrm{CDCl}_{3}\right)$ 管<smiles>Cc1cccc(C)c1N1CC(=O)N2c3ccc(Cl)cc3C(=O)C2(C(=O)N2CC3C=CC2C3)C1=O</smiles>

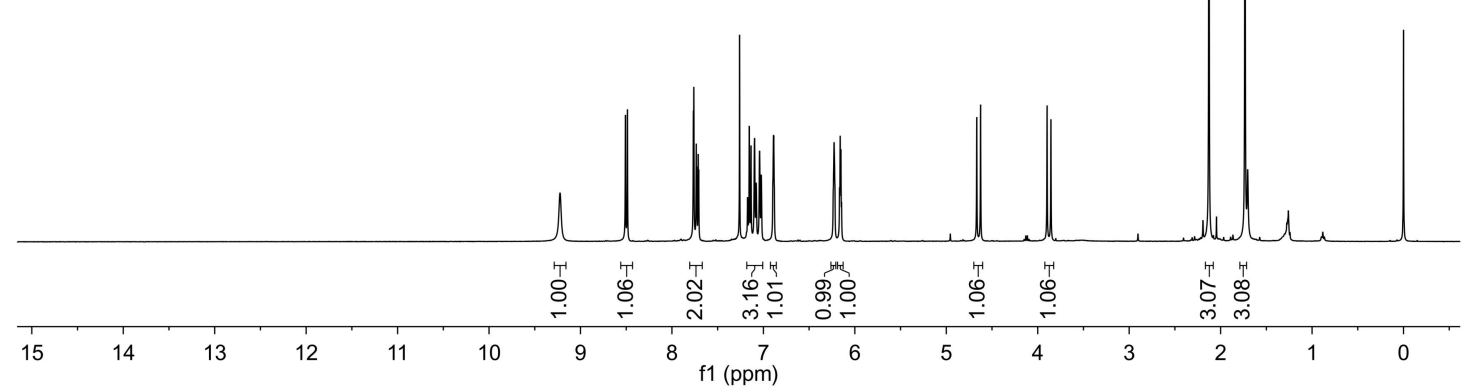

${ }^{13} \mathrm{C}\left\{{ }^{1} \mathrm{H}\right\}$ NMR of Compound 10e $\left(100 \mathrm{MHz}, \mathrm{CDCl}_{3}\right)$

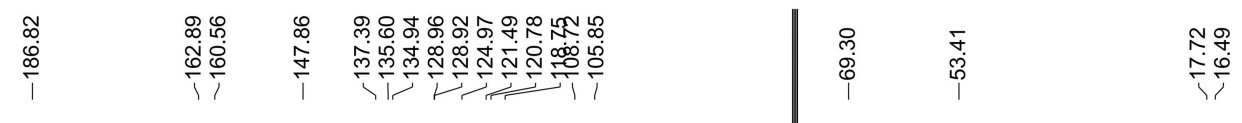

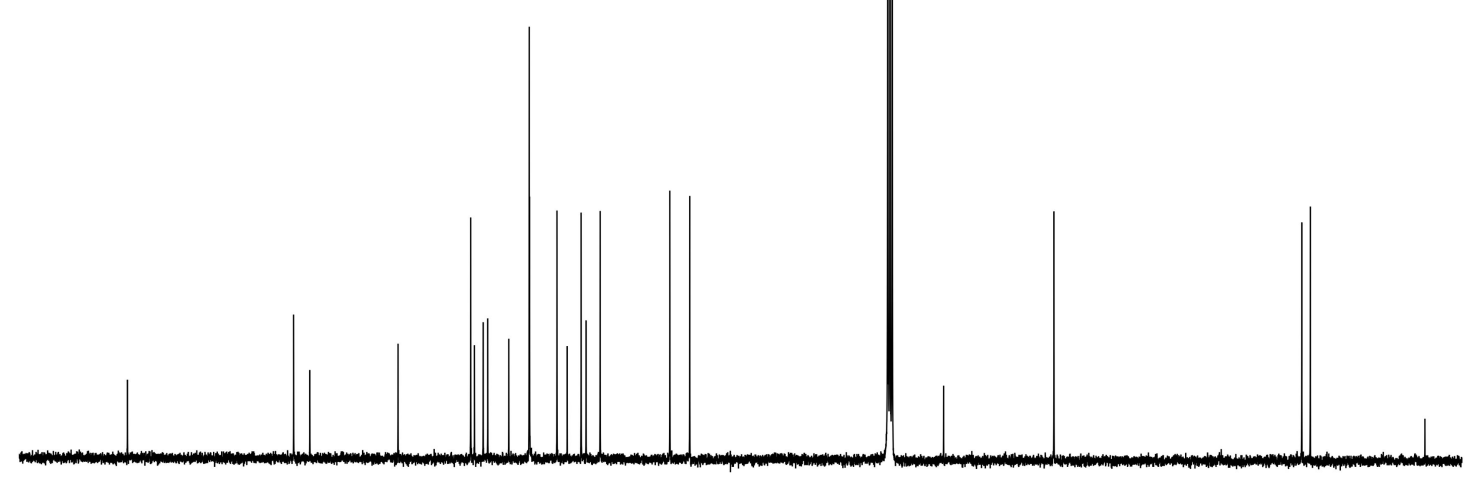

$\begin{array}{lllllllllllllllllllllllllll}200 & 190 & 180 & 170 & 160 & 150 & 140 & 130 & 120 & 110 & 100 & 90 & 80 & 70 & 60 & 50 & 40 & 30 & 20 & 10 & 0\end{array}$ 
${ }^{1} \mathrm{H}$ NMR of Compound $\mathbf{1 0 f}\left(400 \mathrm{MHz}, \mathrm{CDCl}_{3}\right.$ )

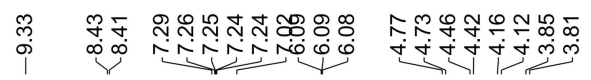

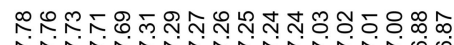

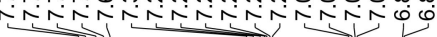
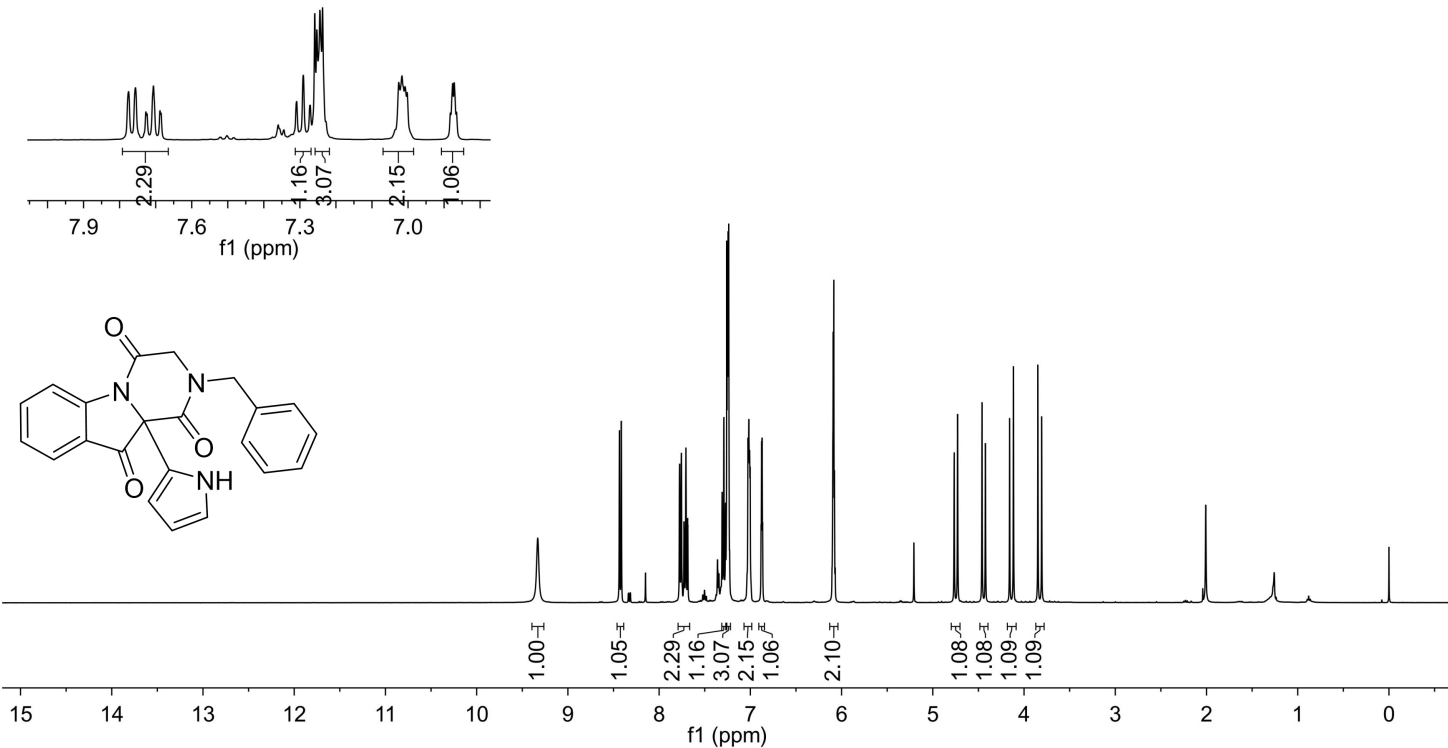

${ }^{13} \mathrm{C}\left\{{ }^{1} \mathrm{H}\right\}$ NMR of Compound $\mathbf{1 0 f}\left(100 \mathrm{MHz}, \mathrm{CDCl}_{3}\right)$

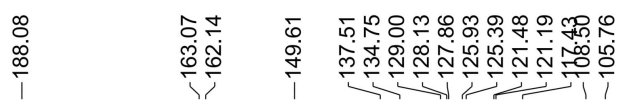

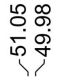

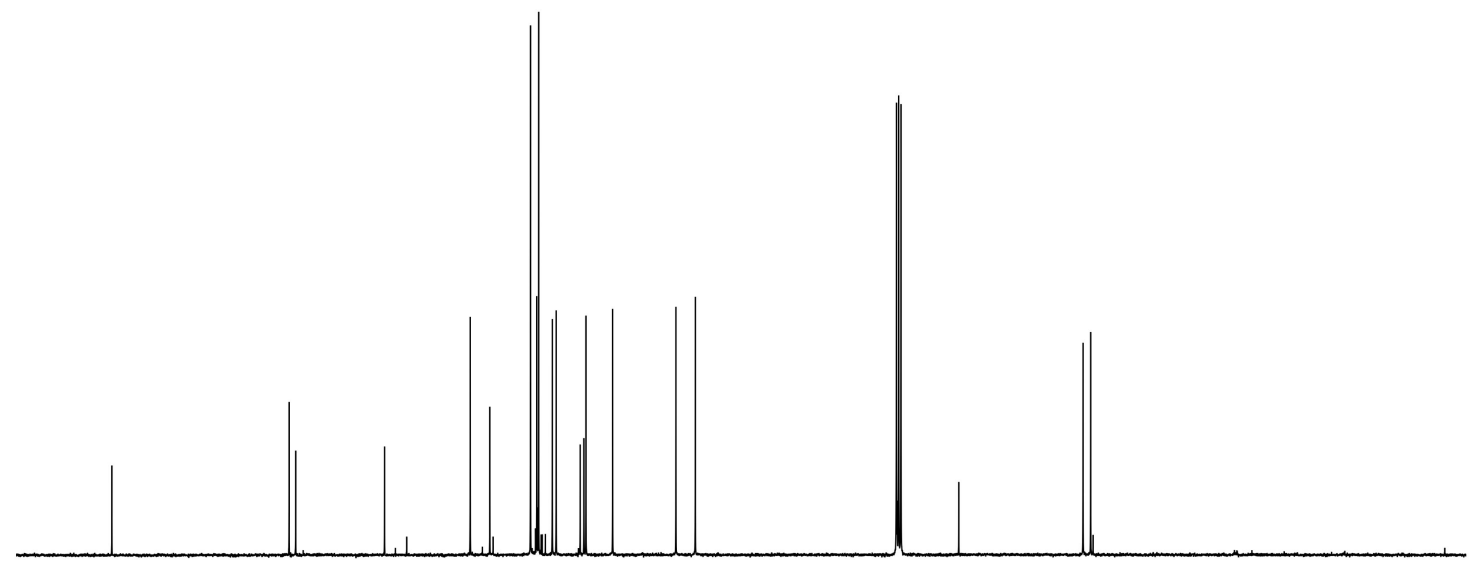

$\begin{array}{lllllllllllllllllllll}200 & 190 & 180 & 170 & 160 & 150 & 140 & 130 & 120 & 110 & \begin{array}{c}100 \\ \mathrm{f} 1(\mathrm{ppm})\end{array} & 90 & 80 & 70 & 60 & 50 & 40 & 30 & 20 & 10 & 0\end{array}$ 
${ }^{13} \mathrm{C}\left\{{ }^{1} \mathrm{H}\right\}$ dept135 NMR of Compound $10 f\left(100 \mathrm{MHz}, \mathrm{CDCl}_{3}\right)$

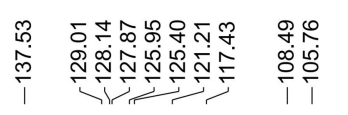

น้ำ
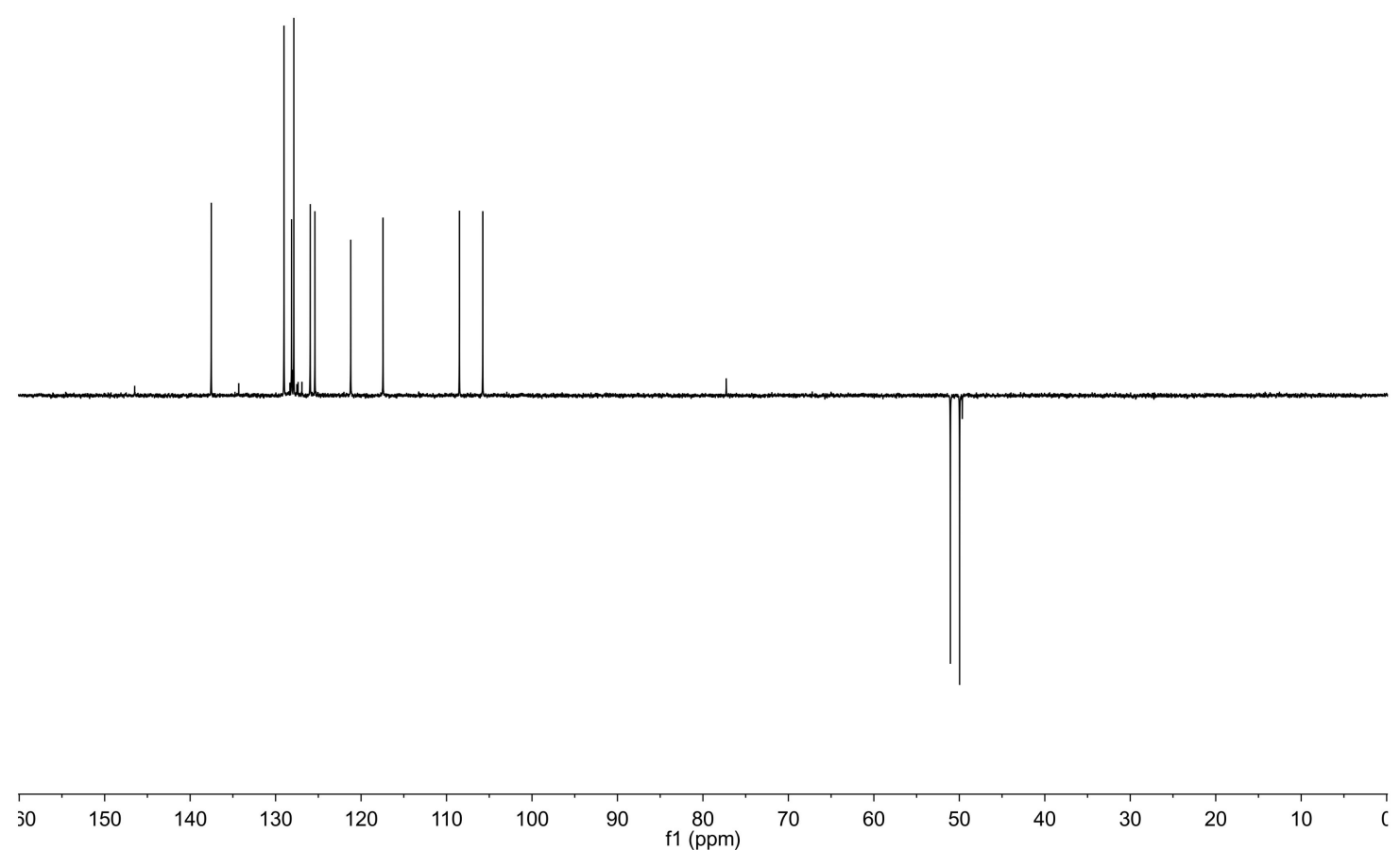

80 
${ }^{1} \mathrm{H}$ NMR of Compound $10 \mathrm{~g}\left(400 \mathrm{MHz}, \mathrm{CDCl}_{3}\right)$

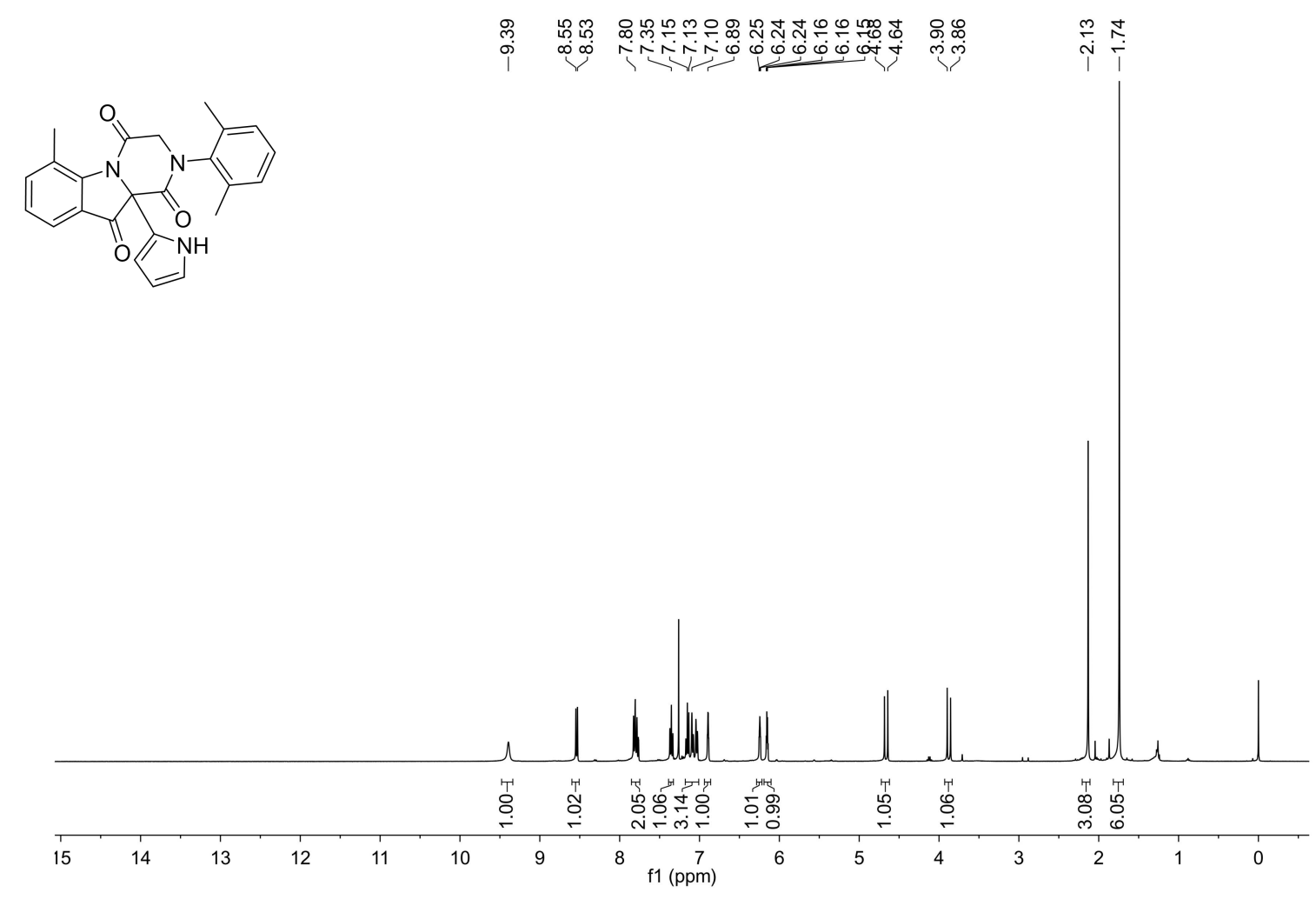

${ }^{13} \mathrm{C}\left\{{ }^{1} \mathrm{H}\right\}$ NMR of Compound $\mathbf{1 0 g}\left(100 \mathrm{MHz}, \mathrm{CDCl}_{3}\right)$

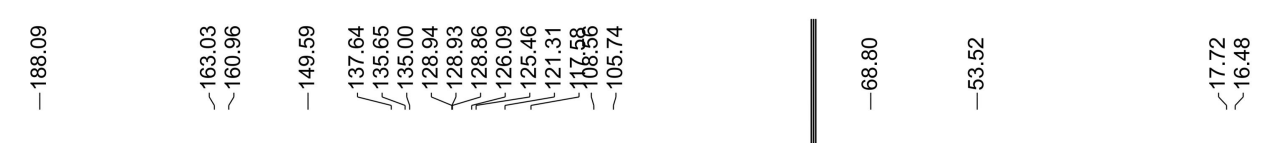

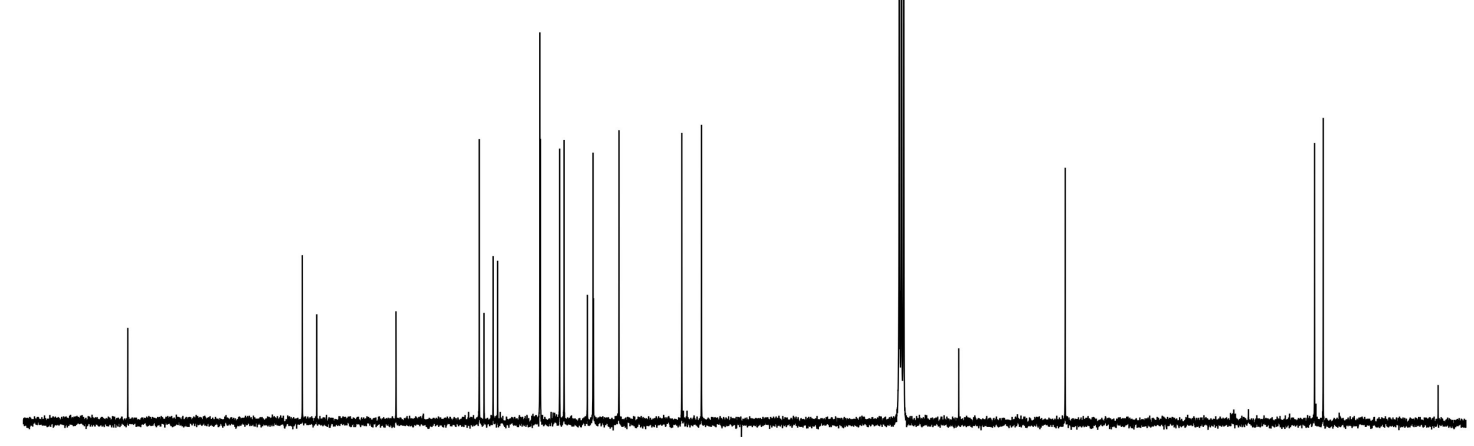

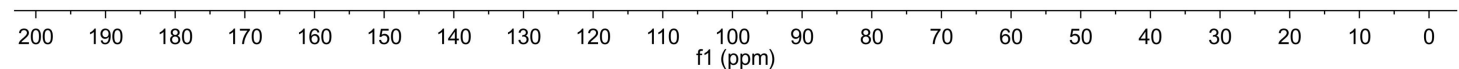


${ }^{1} \mathrm{H}$ NMR of Compound 10h $\left(400 \mathrm{MHz}, \mathrm{CDCl}_{3}\right)$

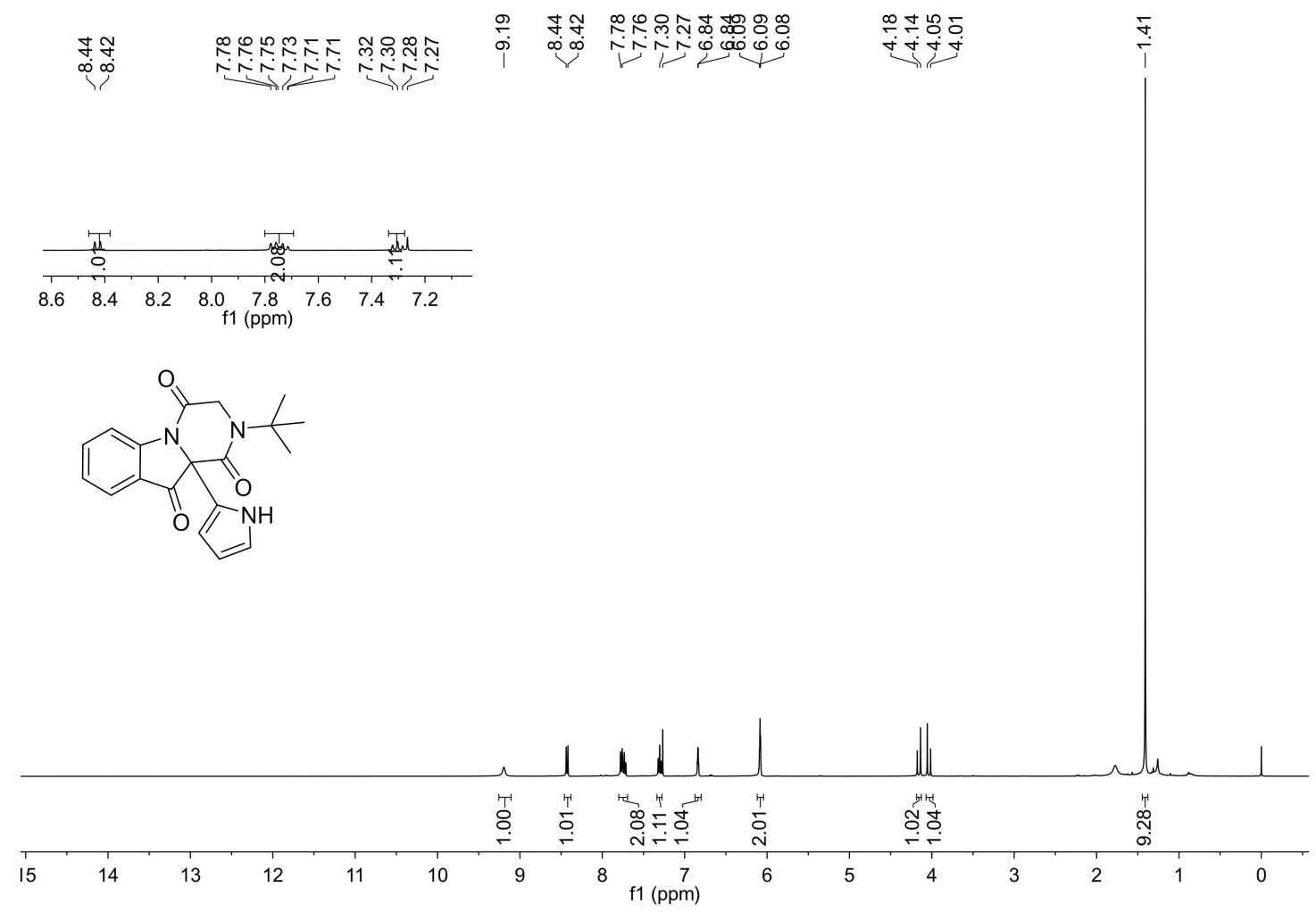

${ }^{13} \mathrm{C}\left\{{ }^{1} \mathrm{H}\right\}$ NMR of Compound $\mathbf{1 0 h}\left(100 \mathrm{MHz}, \mathrm{CDCl}_{3}\right)$

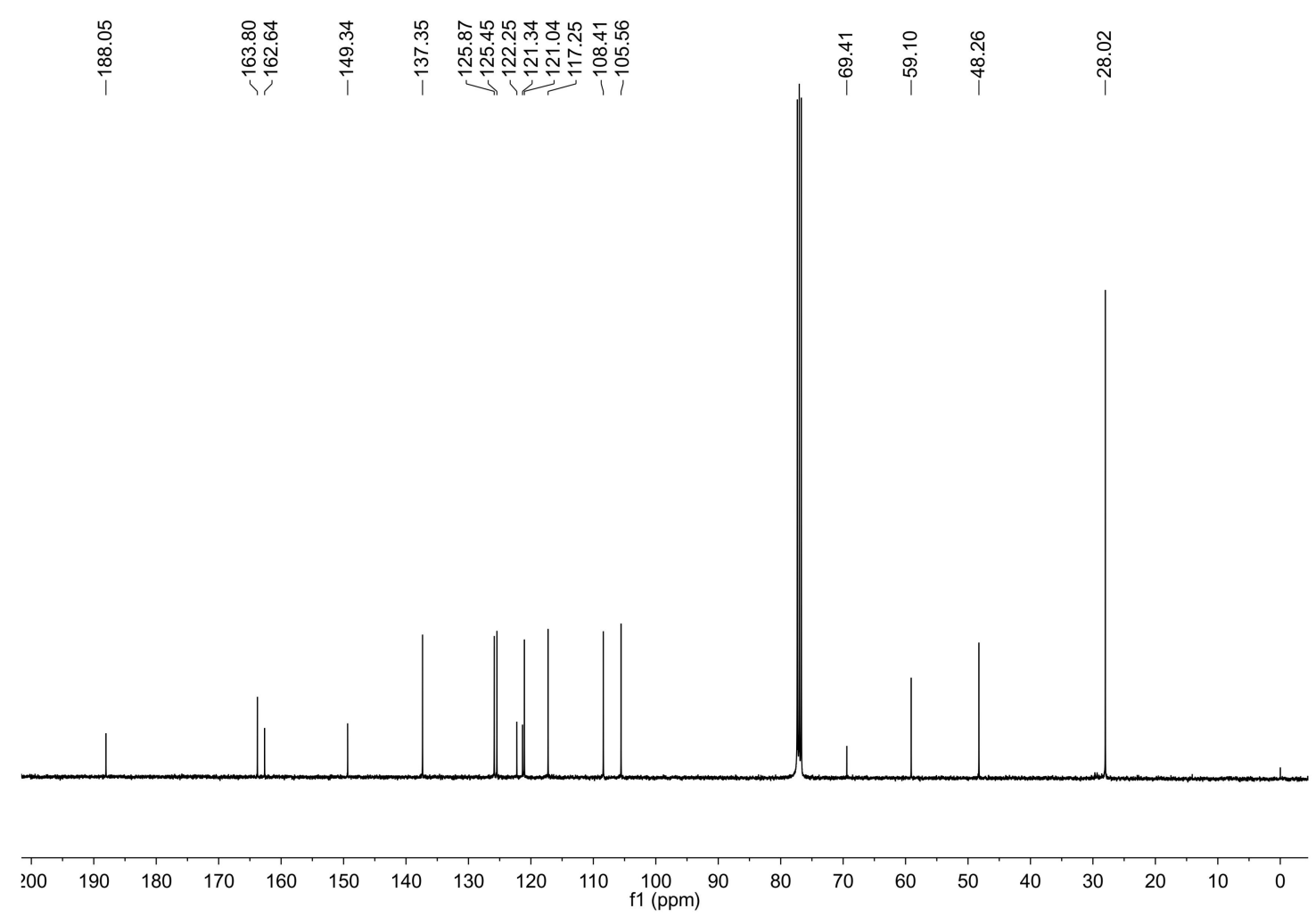


${ }^{1} \mathrm{H}$ NMR of Compound 10i $\left(400 \mathrm{MHz}, \mathrm{CDCl}_{3}\right)$

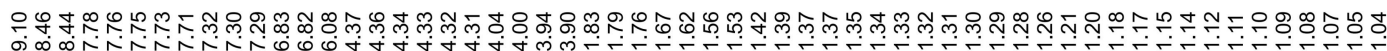<smiles>O=C1CN(C2CCCCC2)C(=O)C2(c3ccc[nH]3)C(=O)c3ccccc3N12</smiles>

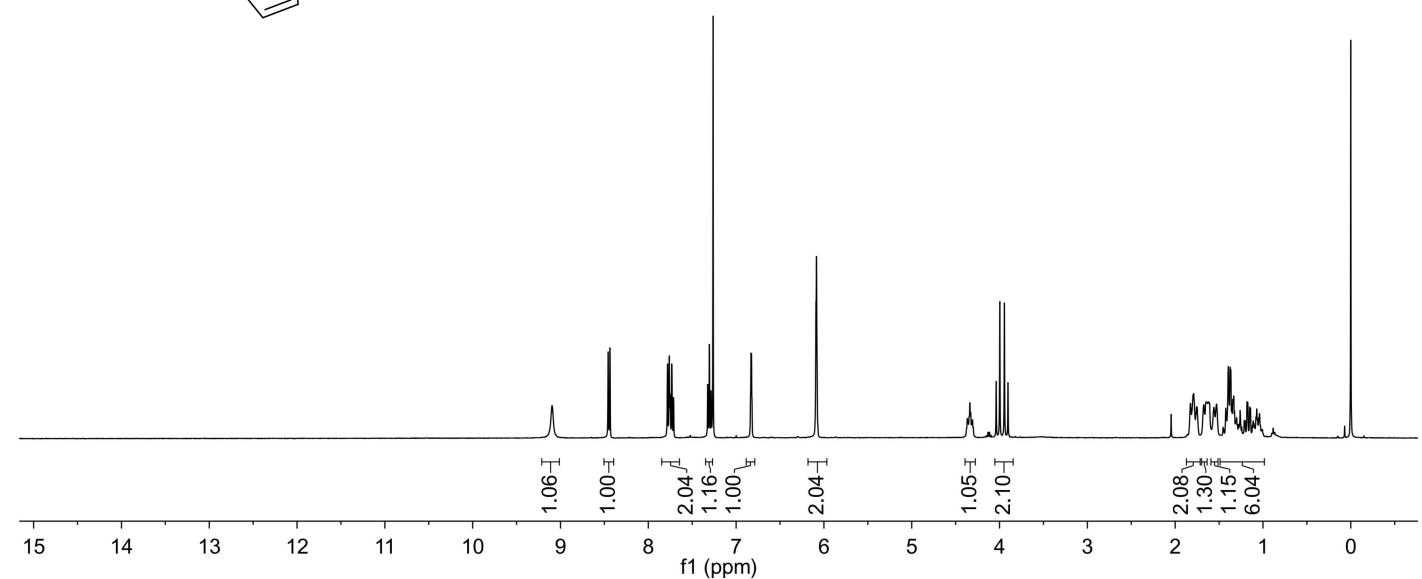

${ }^{13} \mathrm{C}\left\{{ }^{1} \mathrm{H}\right\}$ NMR of Compound 10i $\left(100 \mathrm{MHz}, \mathrm{CDCl}_{3}\right)$

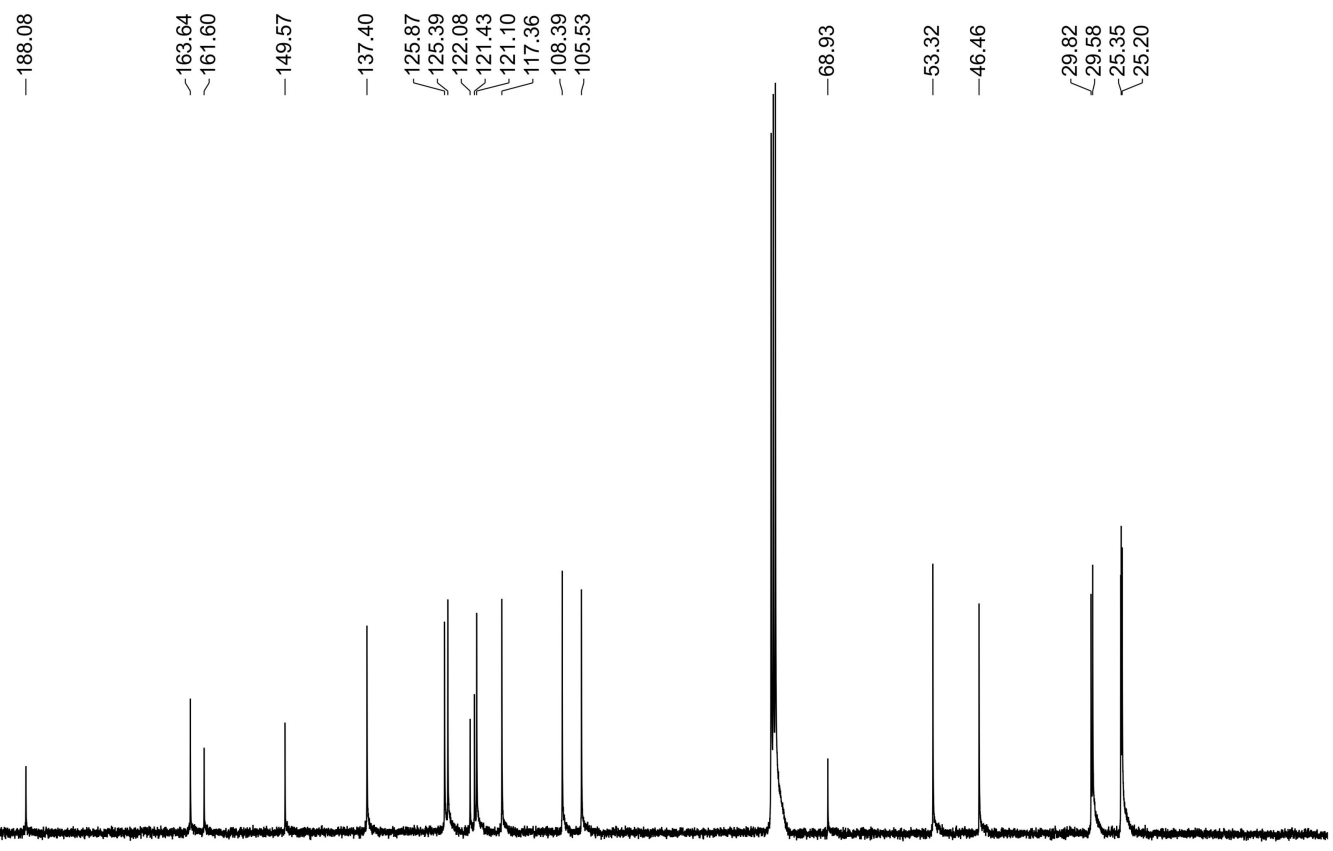

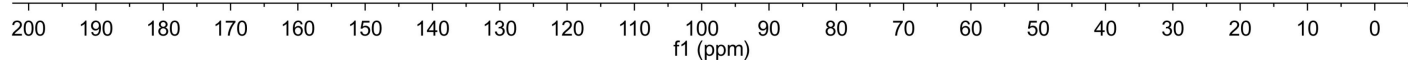


${ }^{1} \mathrm{H}$ NMR of Compound $11\left(400 \mathrm{MHz}, \mathrm{CDCl}_{3}\right)$

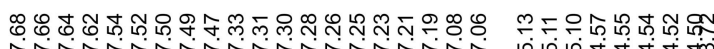

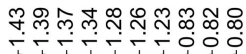

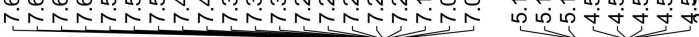

(1)

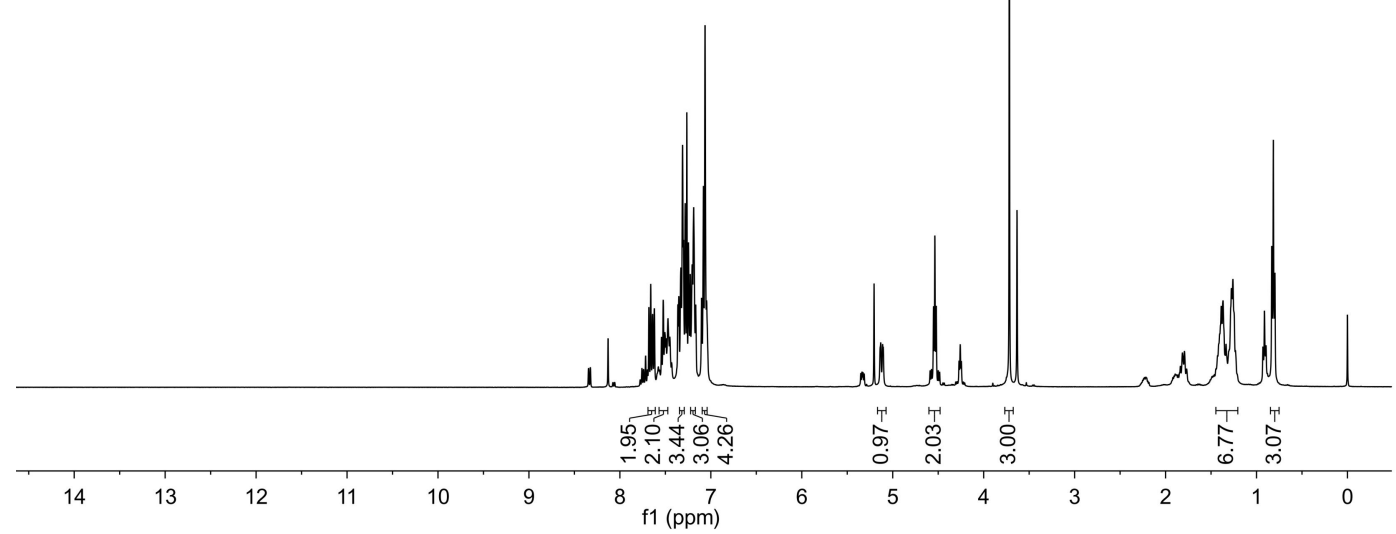

${ }^{13} \mathrm{C}\left\{{ }^{1} \mathrm{H}\right\}$ NMR of Compound $11\left(100 \mathrm{MHz}, \mathrm{CDCl}_{3}\right)$

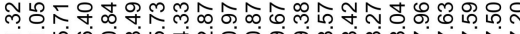

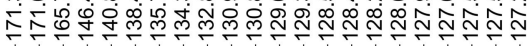

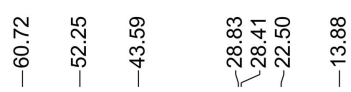

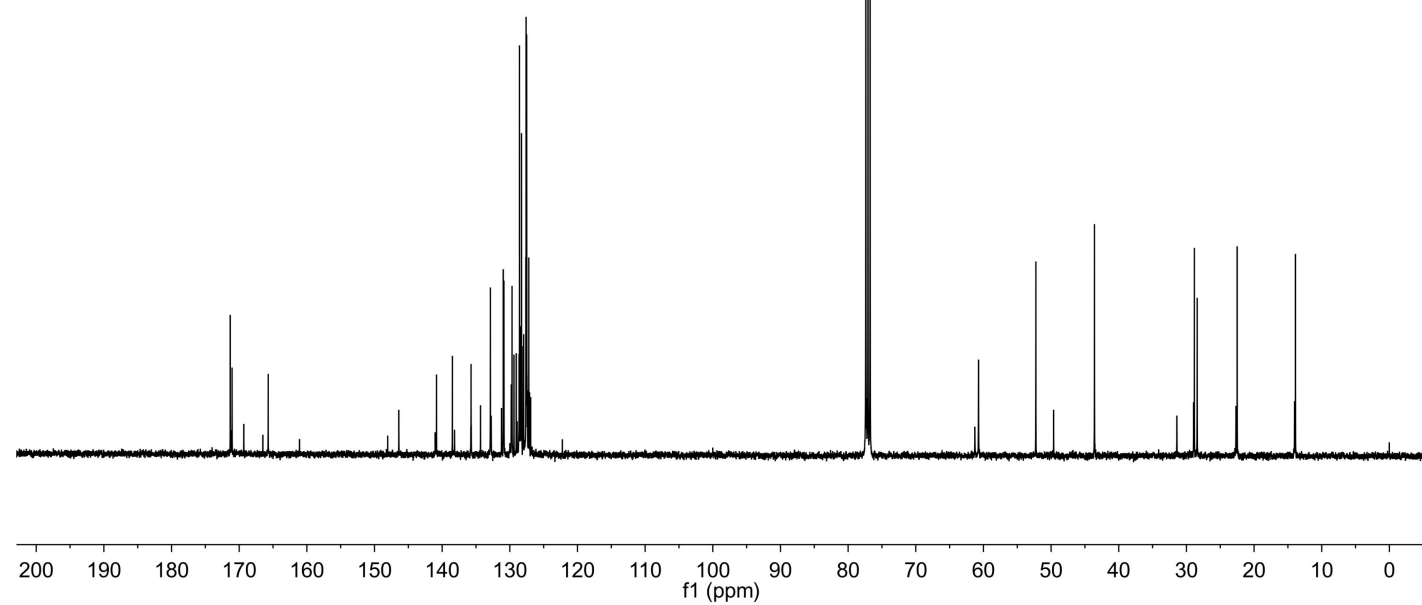


${ }^{1} \mathrm{H}$ NMR of Compound $13\left(400 \mathrm{MHz}, \mathrm{CDCl}_{3}\right)$

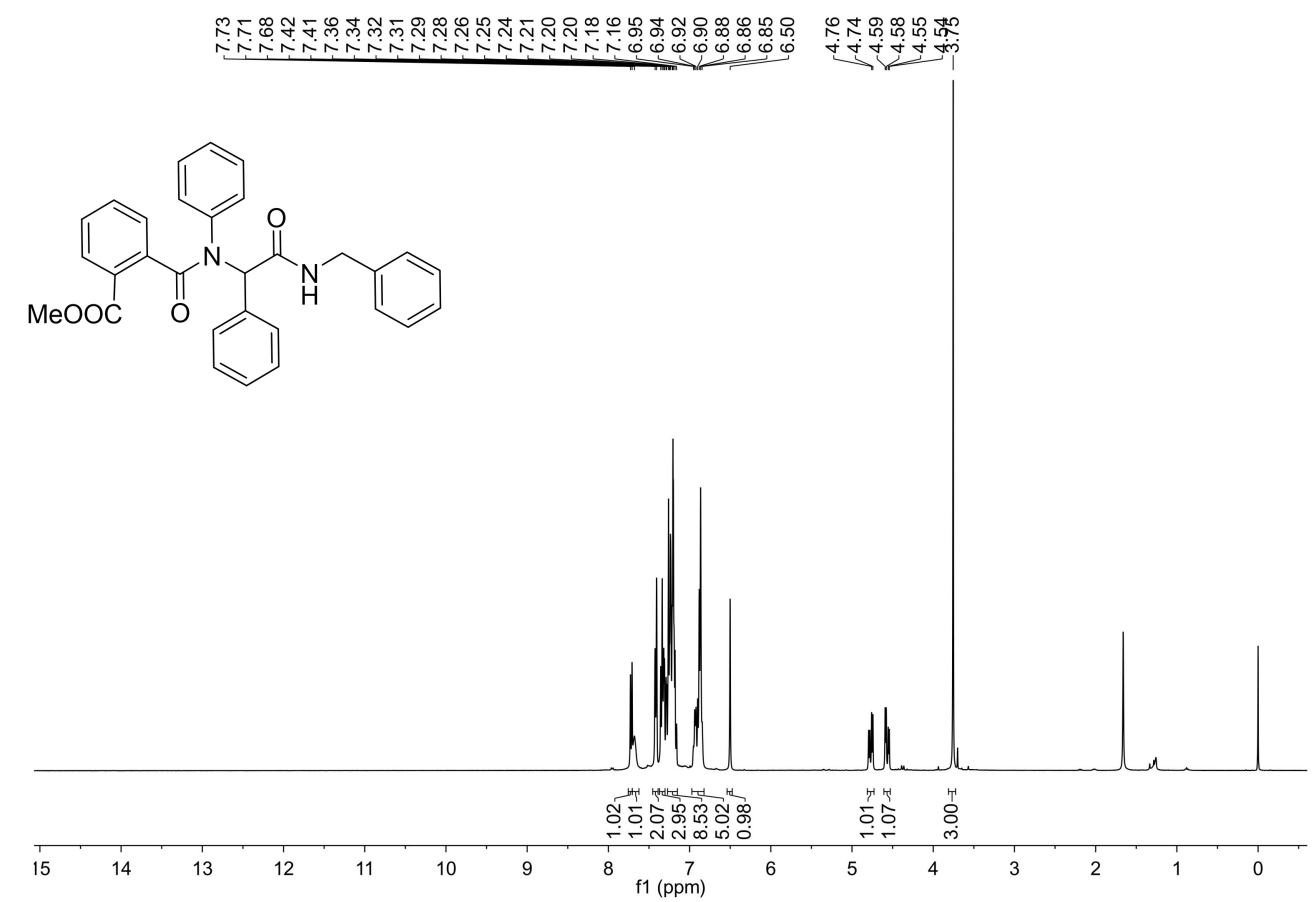

${ }^{13} \mathrm{C}\left\{{ }^{1} \mathrm{H}\right\}$ NMR of Compound $13\left(100 \mathrm{MHz}, \mathrm{CDCl}_{3}\right)$

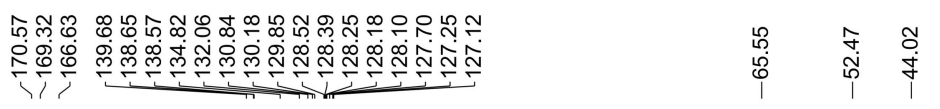

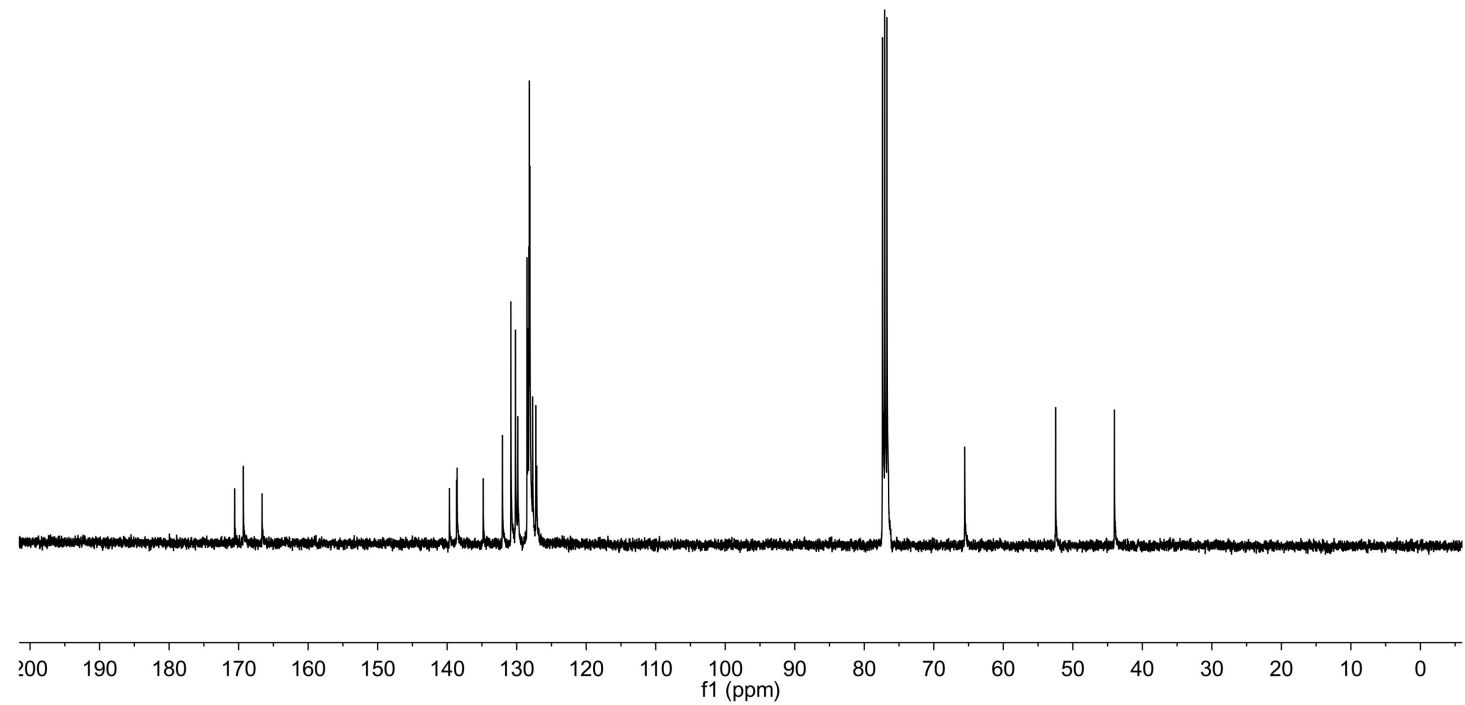

